\title{
AN EFFICIENT METHOD FOR TIME-MARCHING SUPERSONIC FLUTTER PREDICTIONS USING CFD
}

\author{
By \\ JOHN PAUL HUNTER \\ Bachelor of Science \\ Oklahoma State University \\ Stillwater, Oklahoma
}

1994
Submitted to the Faculty of the Graduate College of Oklahoma State University in partial fulfillment of the requirements for the Degree of MASTER OF SCIENCE May, 1997




\section{AN EFFICIENT METHOD FOR TIME-MARCHING SUPERSONIC FLUTTER PREDICTIONS \\ USING CFD}

Thesis Approved:

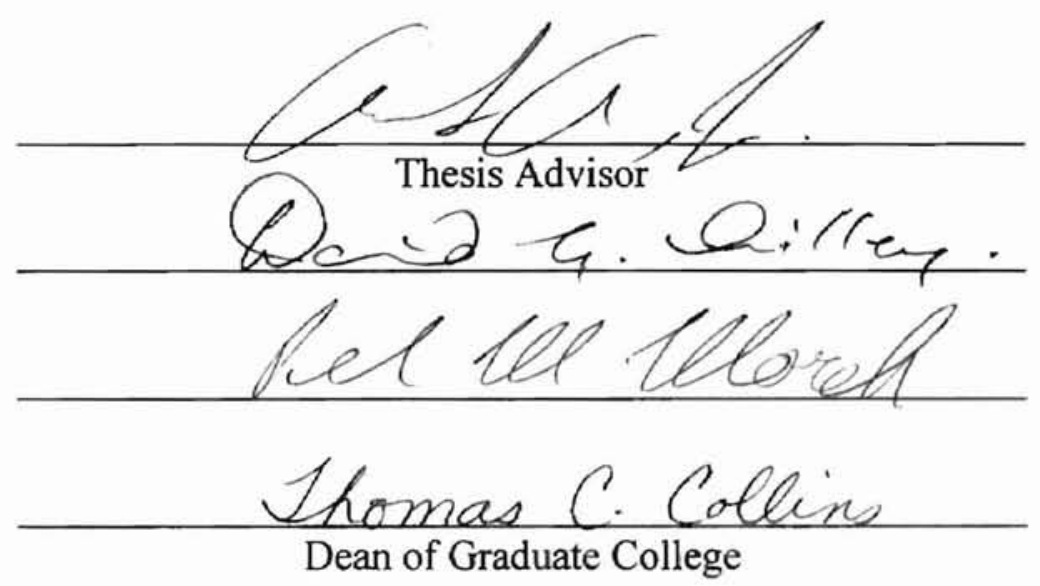




\section{ACKNOWLEDGMENTS}

I would first like to thank the Neumann and Sciara family for instilling at a very young age the many important lessons and positive aspects of life which plays a crucial role in who I am today.

At the age of four I was adopted by a family which had many lessons to teach and much love to give. To the Hunter family I express a deep appreciation for their efforts to help me get where I am today. I would also like to give special thanks to my late grandfather, Charles E. Hunter, who was and still is a great inspiration to me in many ways, not to mention a proud former graduate from OSU.

The staff and professors in the MAE department at OSU are another family which has been a very positive influence not only in the development of my education leading to this point but also in the development of a person as a whole. I would also like to acknowledge the members on my committee, Dr. David G. Lilley, and Dr. Peter M. Moretti, for their continuing efforts to further the completion of my education.

Lastly, I would like to express my sincere appreciation to Dr. Andrew S. Arena for his endless chivalrous effort and devotion to teaching and research. He has not only been an inspiration in academia but also a prominent role model as well. It goes without saying that without the guidance, opportunities and challenges set forth by Dr. Arena, the opportunity to gain experience from this research would not have been made available. 


\section{TABLE OF CONTENTS}

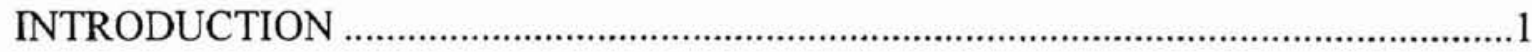

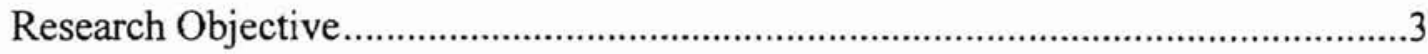

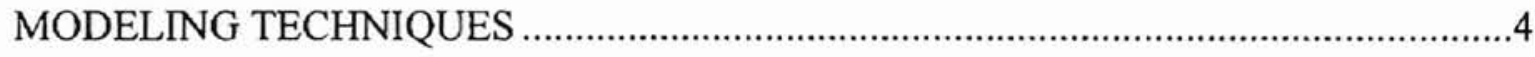

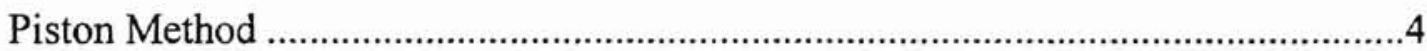

Tangent-Cone Methods ....................................................................................

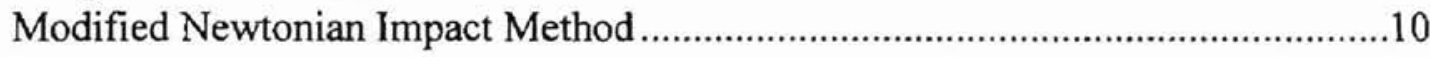

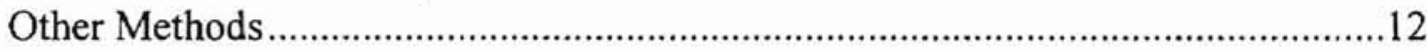

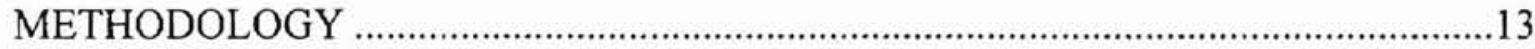

Derivation and Rationale of the Piston Perturbation Method …...............................17

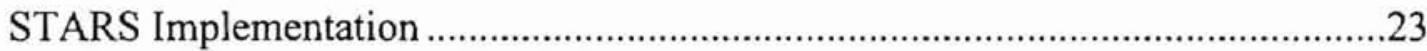

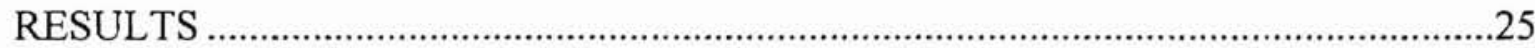

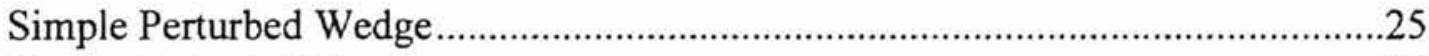

Simple Perturbed Cone......................................................................................... 31

Clamped Plate With Heavy Shock Interaction.........................................................35

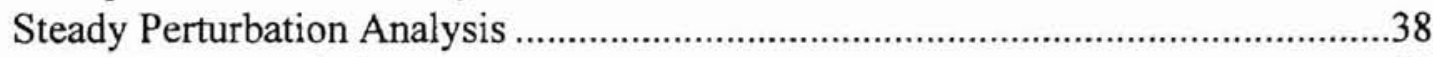

GHV Flutter Analysis....................................................................................40

Cone \& Swept Wing Configuration .....................................................................42 
Conclusions .53

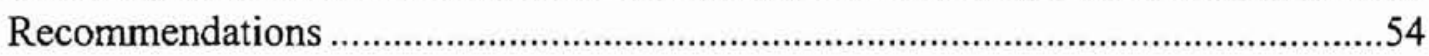

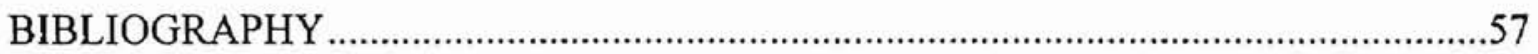

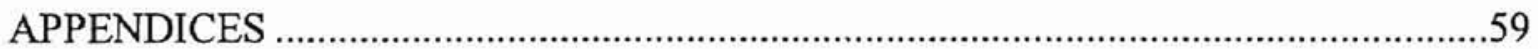

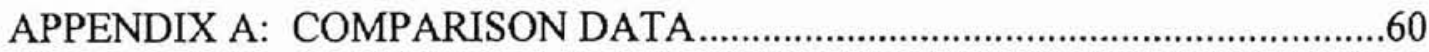

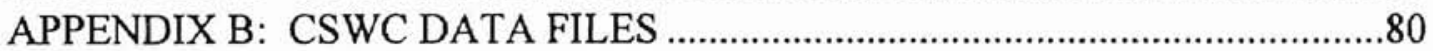

APPENDIX C: MODE 1 TRANSIENT ANALYSIS............................................94 


\section{LIST OF TABLES}

Table

Table 4.1. Point Location for The CSWC. 44

Table 4.2. CSWC Structural Modal Frequencies............................................................45

Table 4.3. CSWC Material Properties ..........................................................................45

Table 4.4. CSWC Flutter Boundary Data .................................................................... 


\section{LIST OF FIGURES}

Figure

Page

Figure 1.1. Graphical Representation Of STARS Modules. .2

Figure 2.1. Piston Motion In A One-Dimensional Channel .5

Figure 2.2. Pressure Coefficient Versus Mach Number For A Wedge And Cone At Half Angles Of $12.5^{\circ}$ Degrees As Applied To Piston Theory 6

Figure 2.3. Percent Error Of Piston Theory Application To The Wedge And Cone 6

Figure 3.1. An Illustration Depicting The Modified Unsteady Wave Equation Applied to A Cone 14

Figure 3.2. $\mathrm{C}_{\mathrm{p}}$ Of A Cone Perturbed About The Mean Flow Of $10^{\circ}$ To $12.5^{\circ}$ .16

Figure 3.3. \% Error Vs. Mach Number For $\mathrm{Cp}$ Of A Cone Perturbed From $10^{\circ}$ To $12.5^{\circ}$ 16

Figure 3.4. Simplistic Illustration Of A Locally Applied Perturbation To The Mean Flow .22

Figure 4.1. A Simple Perturbed Wedge In Compression .26

Figure 4.2. A Simple Perturbed Wedge In Expansion .26

Figure 4.3. More Complete Data Set For A Simple Wedge In Compression 29

Figure 4.4. A More Complete Data Set For A Simple Wedge In Expansion .30 
Figure

Figure 4.5. A Simple Perturbed Cone In Compression 31

Figure 4.6. A Simple Perturbed Cone In Expansion 32

Figure 4.7. A More Complete Data Set For A Simple Cone In Compression .34

Figure 4.8. A More Complete Data. Set For A Simple Cone In Expansion 35

Figure 4.9. A Fixed Rectangular Duct With An Elastically Flexible Clamped Flat Plate 36

Figure 4.10. A Side View Showing The Pressure Contours Of The Heavy Shock Interactions With The Elastic Plate

Figure 4.11. Steady State Deformation Of The Elastic Plate Generated By:

(A) Unsteady Euler Analysis, (B) Piston Theory, (C) Perturbation Method 38

Figure 4.12. Pressure Comparisons At $5^{\circ}$ Angle Of Attack Using Euler, Piston, And Perturbation Method At Three Sectional Cuts On A GHV 39

Figure 4.13. GHV Baseline Surface Mesh .40

Figure 4.14. Flutter Boundary And Run-Time Comparisons At M=2.2 With 705

Time Steps / Transient 41

Figure 4.15. Cone \& Swept Wing Configuration Baseline Mesh 43

Figure 4.16. CSWC Specific Dimensions 43

Figure 4.17. Symmetrical Wing Cross Section. 45

Figure 4.18. Pressure Contours Generated Using Steady Finite Element Euler Analysis at Mach 1.3

Figure 4.19. CSWC Flutter Boundary Analysis For Mach 1.3 
Figure

Figure 4.20. Pressure Contours Generated Using Steady Finite Element Euler Analysis at Mach 1.6 48

Figure 4.21. CSWC Flutter Boundary Analysis For Mach 1.6

Figure 4.22. CSWC Pressure Contours Generated Using Steady Finite Element Euler Analysis (left column) and Flutter Boundary Analysis (right column) 50

Figure 4.23. CSWC Flutter Boundary ………………….........................................51

Figure 4.24. CSWC Flutter Boundary \% Error Analysis................................................51 


\section{NOMENCLATURE}

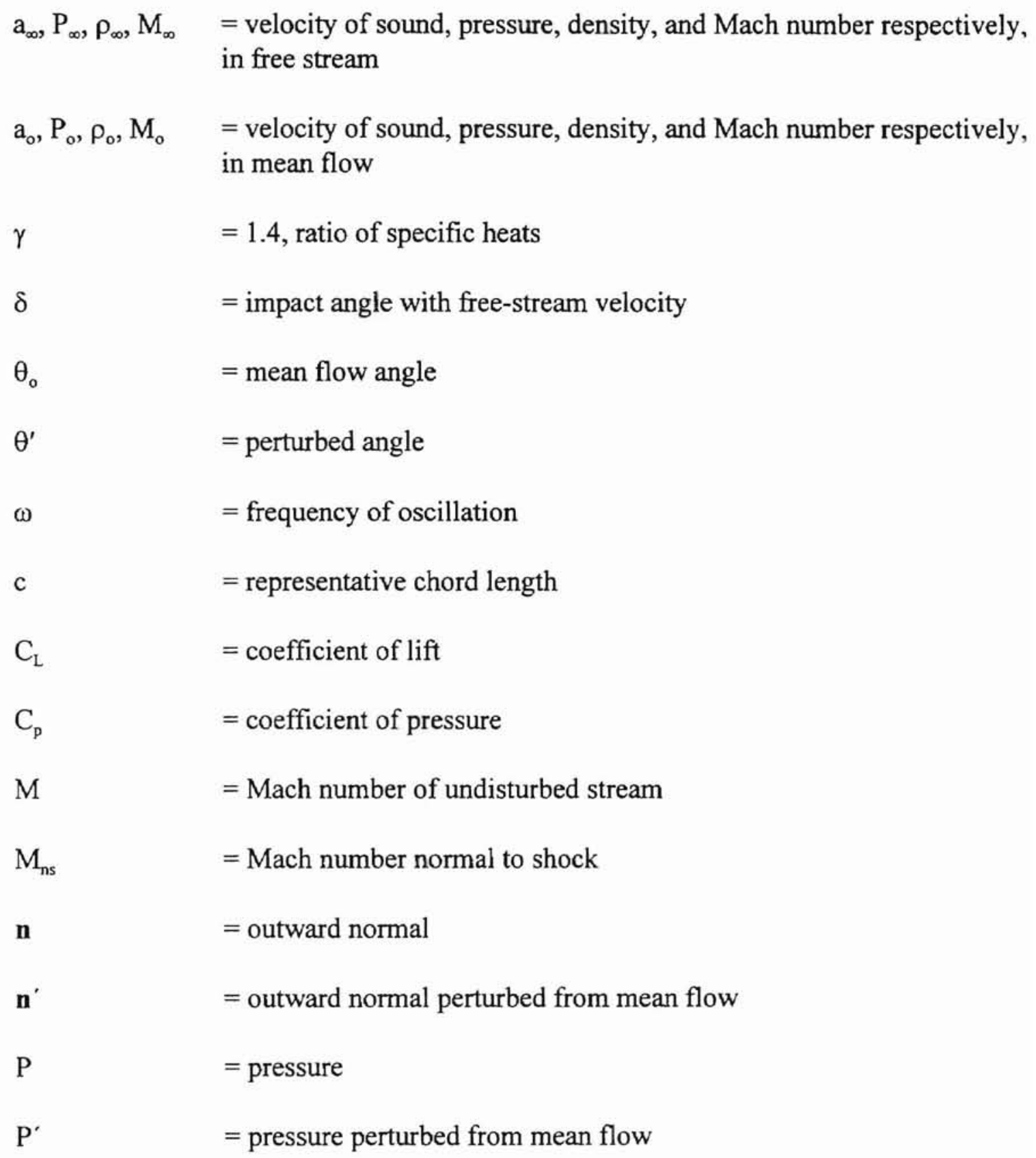


$=$ generalized displacement

$\mathrm{R}$

$=$ ideal gas constant

S

$=$ entropy

$\mathrm{U}$

$=$ velocity of undisturbed stream

$w(t), u(t)$

$=$ piston velocity as a function of time

$\mathrm{V}_{\mathrm{b}}$

$=$ velocity of body

$\mathrm{V}_{\mathrm{s}}$

$=$ local steady velocity 


\section{CHAPTER 1}

\section{INTRODUCTION}

A prediction of aircraft flight dynamics and aeroelastic characteristics such as flutter [Fung, 1969] are crucial to the design of modern aircraft as well as to flight test operations. Using a recently developed STARS [Gupta, 1990] capability for aeroelastic analysis, a time-marching approach based on finite element unsteady Euler analysis may be utilized to predict flutter boundaries over a wide Mach number range for complex three-dimensional geometries. Determination of the flutter boundaries is presently achieved by searching over the flight regime for potential crossovers between stable and divergent time history oscillations based on modal damping terms. This analysis is followed by interpolation of these results to determine the point at which the system is neutrally stable.

STARS which stands for "STructural Analysis RoutineS" was developed by Dr. Kajal K. Gupta at the NASA Dryden Flight Research Center. STARS is a highly integrated computer program for multidisciplinary analysis of flight vehicles including static and dynamic structural analysis, computational fluid dynamics, heat transfer, and 
aeroservoelasticity capabilities. An illustration of the different modules of this program is shown below in Figure 1.1.

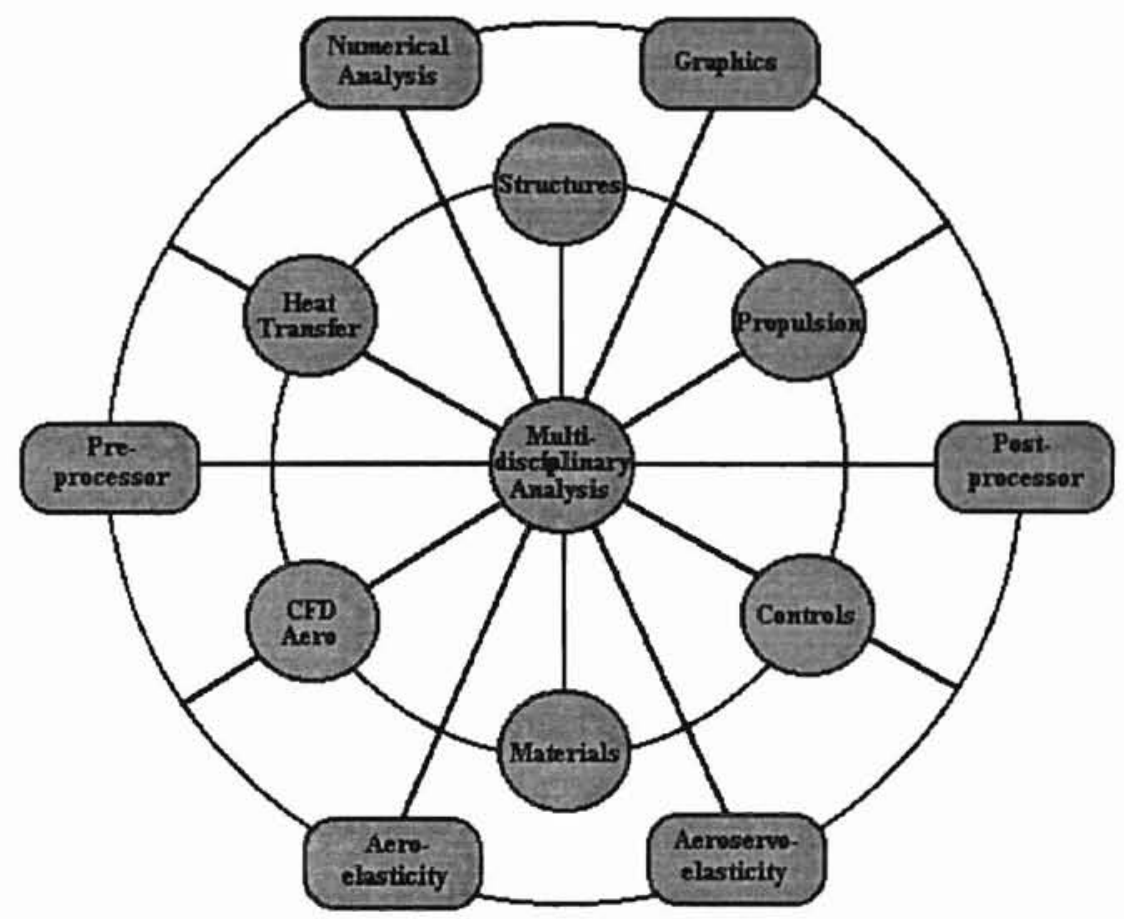

Figure 1.1. Graphical Representation Of STARS Modules

With the recently developed capability for Aeroelastic analysis using a time-marching approach based on the unsteady Euler equations, the aforementioned prediction of flutter boundaries may be obtained for a wide variety of flight conditions and geometries.

Due to the potentially large domain required to ensure sufficient grid resolution of a given geometry, however, there lies a critical drawback of the time-marching approach. Dowel in his book A Modern course in Aeroelasticity points out that the computational time required will be on the order of $\mathbf{P} * \mathbf{T}_{\mathbf{F}}$. Here $\mathbf{P}$ is the number of parameter combinations required and $\mathbf{T}_{\mathbf{F}}$ is the time required for a simultaneous fluid-structure time 
marching calculation to complete a transient. Therefore, in order to ensure time accuracy and sufficient grid resolution, the use of time-marching solutions to the Euler equations on a three-dimensional configuration requires a significant amount of computation time. As an illustration, on a present day high speed workstation, utilization of the unsteady finite element Euler analysis to calculate a single fluid structure transient on a threedimensional system may demand well in excess of one-hundred CPU hours. Hence, identification of the flutter boundaries over the full domain will require many times this number.

Research Objective

Since it is the Computational Fluid Dynamics (CFD) which requires the overwhelming proportion of computation time in time-marching aeroelastic analysis, the focus of this research is to determine a supersonic modeling technique which gives an accurate and expedient estimate of the CFD. Implementation of such a technique will result in significant savings in the computational time required to obtain an aerodynamic solution in the supersonic flow regime. Areas of importance in the determination of such a technique are ease of implementation and compatibility with existing computer codes as well as accuracy over a wide range of geometric shapes and flow regimes. Modeling techniques with these goals in mind are reviewed. 


\title{
CHAPTER 2
}

\section{MODELING TECHNIQUES}

\author{
Piston Method
}

The piston method, used by Lighthill [1953] on oscillating airfoils and later used by Ashley and Zartarian [1956] as an aeroelastic tool, is a popular modeling technique for supersonic and hypersonic aeroelastic analysis. Ashley and Zartarian explains the term "piston theory" as referring to any method for calculating the aerodynamic loads in which the local pressure generated by the body's motion is related to the local normal component of fluid velocity in the same way that these quantities are related at the face of a piston moving in a one-dimensional channel. Ashley also argues that if the piston generates only simple waves and produces no entropy changes, the exact expression for the instantaneous pressure $p(t)$ on its face is depicted by the simple unsteady wave equation shown here in equation 2-1.

$$
p=p_{\infty}\left[1+\frac{\gamma-1}{2} \frac{w}{a_{\infty}}\right]^{\frac{2 \gamma}{\gamma-1}}
$$


Figure 2.1 illustrates this equation.

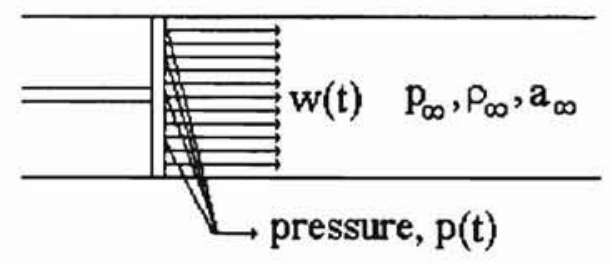

Figure 2.1. Piston Motion In A One-Dimensional Channel

Due to its simplicity and ease of use, the unsteady wave equation is an attractive technique for approximating the surface pressure in a supersonic flow. However, it does not take into account the losses across a shock nor does it accurately predict pressure in an area associated with heavy shock interactions. Furthermore, since this theory is based on a point function, (i.e., the pressures are only dependent upon local conditions), it over predicts the pressure on a three-dimensional geometry such as a cone. This is a result of neglecting the three-dimensional relaxing effect for which the piston theory cannot account. Also, for a relatively blunt surface with respect to the flow, the piston method again over predicts pressure. In Figure 2.2, the unsteady wave equation is used to show the pressure coefficient versus Mach number for a wedge and a cone at half angles of $12.5^{\circ}$ degrees. Since the piston theory does not differentiate between the two geometries one curve represents both geometries. The curve associated with the piston theory is compared with data taken from literature for a cone [Sims, 1964] and wedge [Neice, 1948] at the noted half angle. Figure 2.3 shows the percent error of the noted comparisons. 


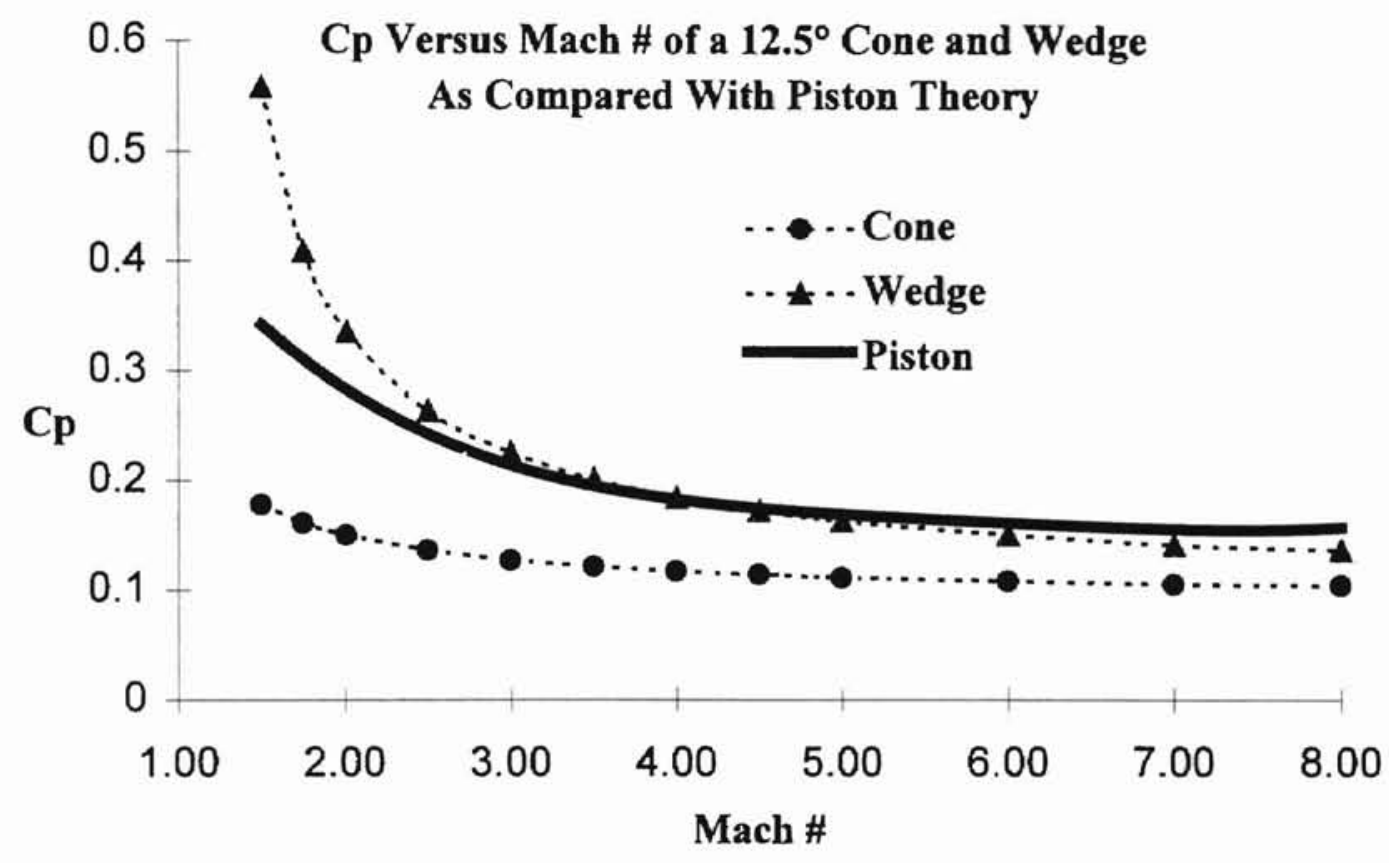

Figure 2.2. Pressure Coefficient Versus Mach Number For A Wedge And Cone At Half Angles Of $12.5^{\circ}$ Degrees As Applied To Piston Theory

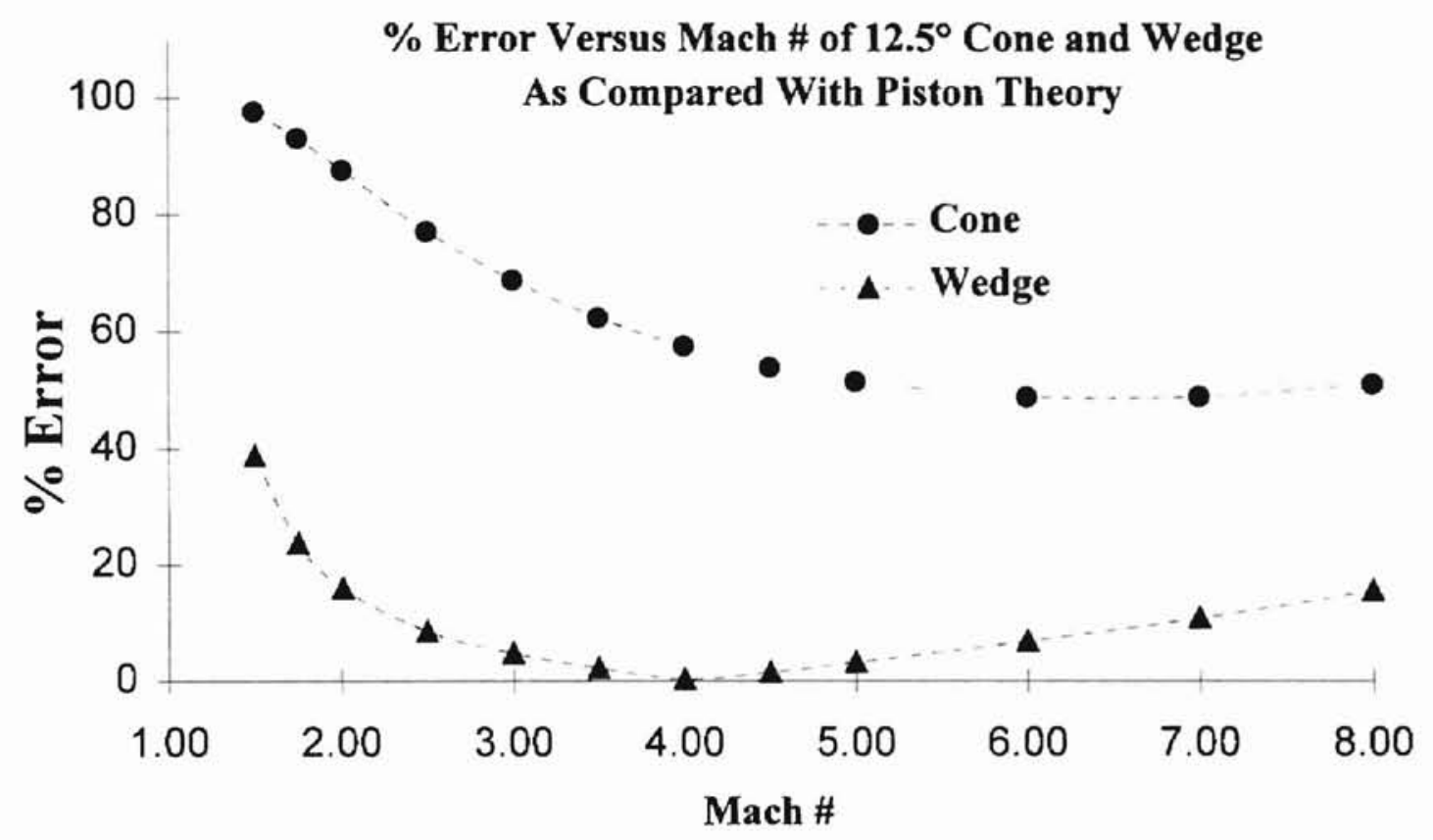

Figure 2.3. Percent Error Of Piston Theory Application To The Wedge And Cone 
Clearly the unsteady wave equation predicts pressure relatively well for a predominately two dimensional flow yet over predicts pressure for three-dimensional flow such as that associated with a cone at the same half angle.

Due to the limited application range and inaccurate prediction of pressure via the Piston theory, a method which will better predict surface pressure about a more threedimensional flow is considered.

Tangent-Cone Methods

In the paper Improved Tangent-Cone Method for the Aerodynamic Preliminary Analysis System (APAS) Version of the Hypersonic Arbitrary-body Program [Cruz \& Sova, 1990], Cruz discusses four impact pressure methods for their ability to predict the zero angle-of-attack inviscid pressure coefficients of sharp cones with angles of varying degrees. These methods are considered for the incorporation as the tangent-cone method into the Aerodynamic Preliminary Analysis System (APAS) [Bonner \& Dunn, 1981], [Pittman, 1979] which uses a modified version of the Hypersonic Arbitrary-Body Program (HABP) Mark III code [Gentry, 1968] in its analysis rationale. The four impact methods evaluated for pressure coefficient prediction were (1) Newtonian theory [Gentry, 1968], (2) the original HABP Mark III tangent-cone empirical method [Gentry, 1968], (3) the Edwards tangent-cone empirical method [Pittman, 1979], and (4) a combination of second-order slender-body theory and the approximate cone solution of Hammit and Murthy [1959]. 
The Modified Newtonian theory yields a pressure coefficient as a function only of the impact angle:

$$
C_{p}=K \cdot \sin ^{2} \delta
$$

where $\mathrm{K}$ is equal to the stagnation-point pressure coefficient [Truitt, 1959].

Both the HABP Mark III and the Edwards version on the tangent-cone empirical method calculate pressure coefficient as a function of Mach number and impact angle:

$$
C_{p}=\frac{48 \cdot M_{n s}^{2} \cdot \sin ^{2} \delta}{23 \cdot M_{n s}^{2}-5}
$$

Cruz further states that the difference between these two methods lies in the empirical equations for Mach number normal to the shock. It is shown that for the HABP Mark III version,

$$
M_{n s}=1.090909 M_{\infty} \sin \delta+e^{-1.090909 M_{\infty} \sin \delta}
$$

For the Edwards version,

$$
M_{n s}=\left(0.87 \cdot M_{\infty}-0.544\right) \cdot \sin \delta+0.53
$$


The last method Cruz discusses uses a combination of second-order slender-body theory and the approximate cone solution of Hammitt and Murthy [1959]. The pressure coefficient found by this method is given by:

$$
C_{p}=\frac{p_{\infty}}{1 / 2 \rho_{\infty} V_{\infty}^{2}}\left(\left(\frac{2 \gamma}{\gamma+1} M_{\infty}^{2} \sin ^{2} \theta_{s}-\frac{\gamma-1}{\gamma+1}\right) \cdot\left(1+\frac{\gamma M_{\infty}\left(\theta_{s}-\theta_{c}\right)^{2} \cos ^{2} \theta_{s}}{1+[(\gamma-1) / 2] M_{\infty}^{2} \sin ^{2} \theta_{s}}\right)^{-1}\right)
$$

It is shown that the Modified Newtonian Impact theory under predicts throughout the conical pressure coefficient throughout the entire Mach range (0-25), whereas the Mark III HABP tangent-cone empirical method does a better job from Mach 5 to 25 yet significantly under predicts below this range. The last two methods which are the Edwards tangent cone empirical method and the " 2 nd Order Slender Body + Hammitt / Murthy" method show much better results as compared with the previous two methods. Since these results are relatively identical except for extreme cases which are not applicable for this research objective, the simpler of the two methods (Edwards corrected tangent-cone method) is used for validation and experimentation. Had this method proved feasible, the more accurate method would have been researched.

Analysis was done on the above tangent-cone method and proven extremely accurate over a wide range of Mach numbers for conical flow as shown in the literature. But since this method's application is for predominately cone shaped geometries, it under predicts pressure for mostly two-dimensional flow and again over predicts pressure for flows about blunt surfaces such as the leading edge of a wing or the rounded nose of a 
hypersonic vehicle. Furthermore, as in the Piston Method, the Tangent-Cone method neglects the effects of the mean flow as well as any shock interactions.

\section{Modified Newtonian Impact Method}

In contrast to the unsteady wave equation and the tangent-cone methods, the Modified Newtonian Impact [Bonner \& Cleaver, 1981] method is used predominately for the prediction of surface pressure on blunt objects in hypersonic conditions. The equation for this theory is briefly discussed in the previous section and further analyzed here:

Anderson [315], in his book, Modern Compressible Flow, discusses that in Propositions 34 and 35 of Isaac Newton's Principia, the force of impact between a uniform stream of particles and a surface is obtained from the loss of momentum of the particles normal to the surface. Newton's assumption is that upon impact with a given surface, the normal momentum of the particle is transferred to the surface, whereas the tangential momentum is preserved. Hence the time rate of change of momentum of this mass flux, from Newton's analysis is as follows:

$$
\text { (Mass flux) } x \text { (velocity change })=\left(\rho V_{\infty} A \sin \theta\right)\left(V_{\infty} \sin \theta\right)=\rho V_{\infty}^{2} A \sin ^{2} \theta
$$

And of course, from Newton's second law, this time rate of change of momentum is equal to the force $\mathrm{F}$ on the surface: 


$$
\frac{F}{A}=\rho V_{\infty}^{2} \sin ^{2} \theta
$$

After non-dimensionalization and rearrangement, the following equation shows the Newtonian impact relation or the newtonian "sin-squared" law for the pressure distribution on a surface inclined at an angle $\theta$ with respect to the free stream:

$$
C_{p}=2 \cdot \sin ^{2} \theta
$$

This equation is said to give a relatively good estimate of the pressure coefficient, however, in 1955, Lester Lees, a professor at the California Institute of Technology, proposed a "modified newtonian" pressure law. Lees suggested that the above equation be modified by replacing the coefficient " 2 " with $\mathrm{C}_{p(\max )}$ which is the pressure at the stagnation point equal to the total pressure behind a normal shock wave at $M_{\infty}$. [Anderson 316]. The equation for $C_{p(\max )}$ is listed below.

$$
C_{p_{\max }}=\left(p_{o}-p_{\infty}\right) / \frac{1}{2} \rho_{\infty} V_{\infty}^{2}
$$

It is discussed by Anderson that this modified pressure law is now in wide use for estimating pressure distributions over blunt surfaces at high Mach numbers and is more accurate than equation the original equation. 
Since however, this method has a limited application of mainly blunt surfaces at high supersonic to hypersonic conditions, other techniques are once again considered.

\section{Other Methods}

The previously noted theories; Piston, Tangent Cone, and Modified Newtonian Impact, are just a few of the many aerodynamic modeling techniques used to predict surface pressure in the supersonic and hypersonic flow regime. The Modified Newtonian Plus Prandtl-Meyer Method is yet another blunt body technique based on the analysis presented by Kaufman found in [Bonner, 1981]. Also noteworthy is the Van Dyke Unified Method found in [Bonner, 1981] useful for thin profile shapes. Conversely, these techniques, among many others, are usually applied to specific cases and tend to be too specific for the task at hand. 


\section{CHAPTER 3}

\section{METHODOLOGY}

Since the full steady finite element Euler analysis is available via STARS as well as many previously noted modeling techniques, the question arises; how may the accuracy and generality of STARS be utilized in conjunction with the efficiency of a modeling technique? Hence, after careful examination and analysis of several supersonic and hypersonic methods, the Piston method is chosen as the most feasible aerodynamic modeling technique. This technique will be utilized as a small perturbation to the existing mean flow solution obtained by the finite-element Euler methodology, hence the "Piston Perturbation Method" or "Perturbation Solution" (P.S.). A simplistic illustration of this concept utilizing the same $12.5^{\circ}$ cone used to generate Figure 2.2, is as follows: First, shock expansion theory (used in place of the finite-element Euler solution) is applied as the mean flow solution for a cone at a half angle of $10^{\circ}$. Next, the unsteady wave equation is modified and applied as a $2.5^{\circ}$ perturbation to the mean flow. 
Figure 3.1 illustrates this equation as it applies to a cone.

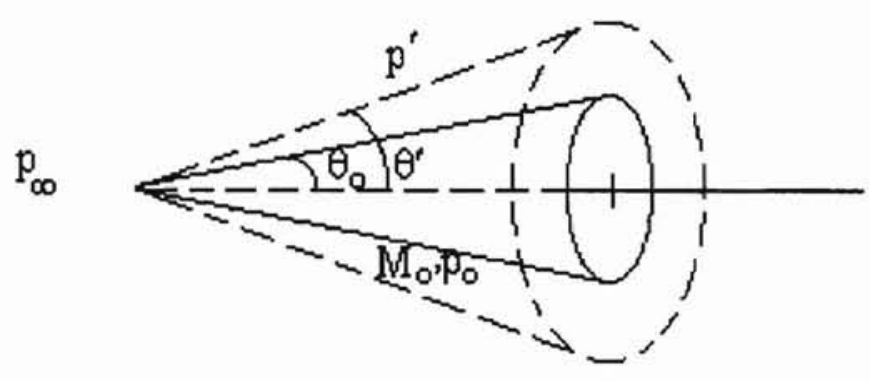

Figure 3.1. An Illustration Depicting The Modified Unsteady Wave Equation Applied to A Cone

The perturbed pressure, $P^{\prime}$, is solved with respect to $P_{\infty}$ as follows,

$$
\frac{P^{\prime}}{P_{\infty}}=\frac{P_{o}}{P_{\infty}} \frac{P^{\prime}}{P_{o}}
$$

The unsteady wave equation is applied as a small perturbation to the mean flow which in this case is $\frac{P_{o}}{P_{\infty}}$,

$$
\frac{P^{\prime}}{P_{\infty}}=\frac{P_{o}}{P_{\infty}}\left[1+\frac{\gamma-1}{2} \frac{\Delta u}{a_{0}}\right]^{\frac{2 \gamma}{\gamma-1}}
$$

Since the rate of change of the body or $V_{b}$ is not taken into account in this example, $\Delta u=V_{o} \cdot \hat{n}=M_{o} a_{o} \operatorname{Sin}\left(\theta^{\prime}-\theta_{o}\right)$. Upon the substitution of $\Delta u$ in the previous equation, 
the following equation results which gives the perturbed pressure ratio across the shock as a function of Mach number and impact angle.

$$
\frac{P^{\prime}}{P_{\infty}}=\frac{P_{o}}{P_{\infty}}\left[1+\frac{\gamma-1}{2} M_{o} \operatorname{Sin}\left(\theta^{\prime}-\theta_{o}\right)\right]^{\frac{2 \gamma}{\gamma-1}}
$$

Since the unsteady wave equation is applied as a small perturbation to the mean flow at $10^{\circ}$, the three-dimensional relaxing effects associated with conical flow are taken into account. This improves the prediction of the surface pressure considerably as compared with results shown in Figure 2.2 and Figure 2.3. Figure 3.2 and Figure 3.3 illustrate the improved results. Be sure to note that the percent error for the unperturbed case discussed in the previous chapter averages about 65 percent whereas the percent error for the P.S. averages approximately 5 percent. The perturbation solution (P.S.) clearly improves the prediction of the surface pressure for conical flow.

The Modified Newtonian method, Tangent-Cone method, as well as the piston method were all analyzed as a small perturbation to a given mean flow over a wide range of Mach numbers and geometries. Hence, it is clearly shown in Appendix A on page 60 in numerous graphs that the piston theory is the most feasible modeling technique for implementation into STARS due to its generality and accuracy over these ranges. 


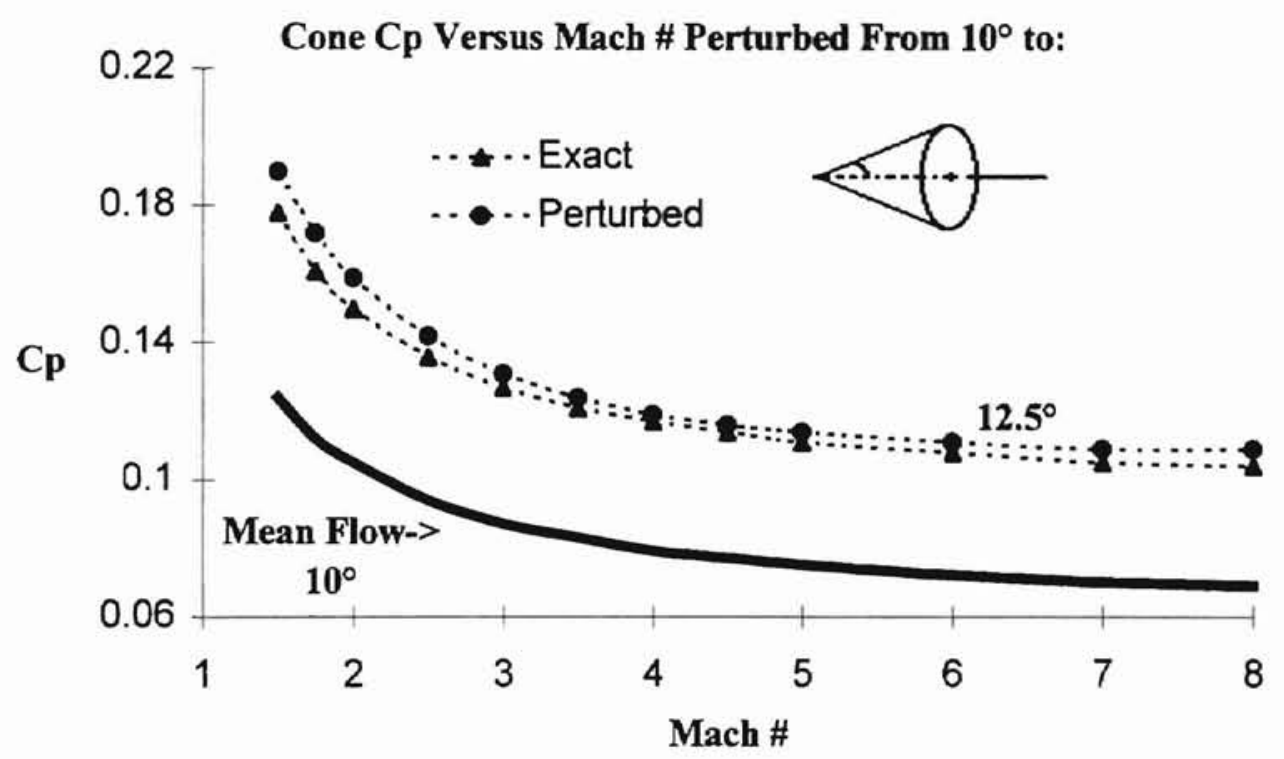

Figure 3.2. $\mathrm{C}_{\mathrm{p}}$ Of A Cone Perturbed About The Mean Flow Of $10^{\circ}$ To $12.5^{\circ}$

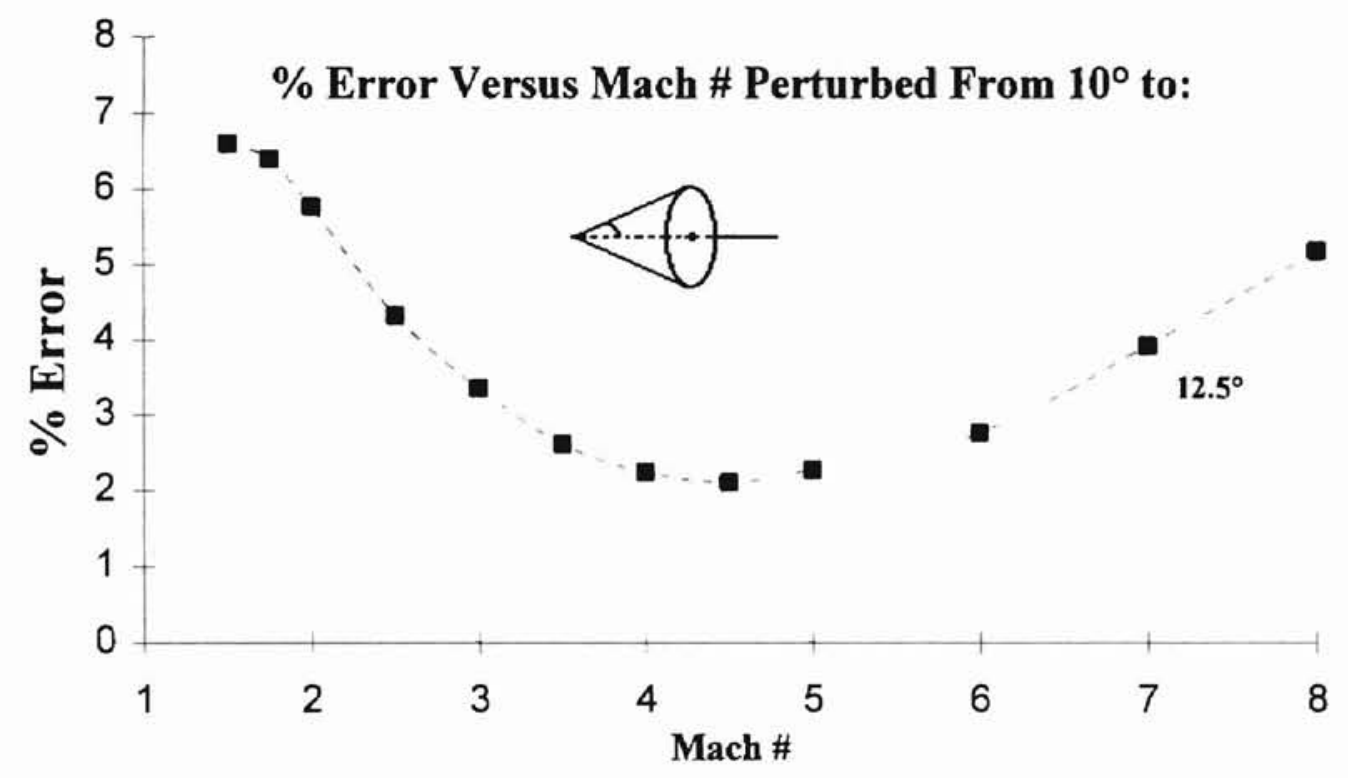

Figure 3.3. \% Error Vs. Mach Number For Cp Of A Cone Perturbed From $10^{\circ}$ To $12.5^{\circ}$ 


\section{Derivation and Rationale of the Piston Perturbation Method}

Since the piston method is the chosen supersonic modeling technique to be used as a perturbation to the steady finite element Euler solution for aeroelastic analysis, a full understanding of this method is necessary. This will be done by first discussing the derivation of the unsteady wave equation. Next, the valid assumptions of this method by Lighthill [1952] and Ashley [1956] are discussed. And finally, rational for the unsteady wave equation to be used as a small perturbation to the mean flow to improve the prediction of the pressure along a given surface is discussed.

As previously noted by Ashley [1956] in chapter 2, it is assumed that if a piston generates only simple waves and produces no entropy changes, the exact expression for the instantaneous pressure $p(t)$ on its face is depicted by the simple unsteady wave equation. A brief derivation of this equation taken from Anderson [190] is as follows:

To develop the governing equations for a finite wave, the continuity equation in the following form is first considered:

$$
\frac{D \rho}{D t}+\rho(\vec{\nabla} \bullet \vec{V})=0
$$

Also, from thermodynamics, $\rho=\rho(p, s)$. Therefore, 


$$
d \rho=\left(\frac{\delta \rho}{\delta p}\right)_{s} d p+\left(\frac{\delta \rho}{\delta s}\right)_{p} d s
$$

And since $d s=0$ for isentropic flow Equation (3-5) becomes,

$$
\frac{D \rho}{D t}=\frac{1}{a^{2}} \frac{D p}{D t}
$$

By substitution of Eq. (3-6) into Eq. (3-4), the following equation is generated:

$$
\frac{1}{a^{2}} \frac{D p}{D t}+\rho(\vec{\nabla} \cdot \vec{V})=0
$$

The previous equation written for one-dimensional flow becomes,

$$
\frac{1}{a^{2}}\left(\frac{\delta p}{\delta t}+u \frac{\delta p}{\delta x}\right)+\rho \frac{\delta u}{\delta x}=0
$$

The momentum equation without body forces is now considered:

$$
\rho \frac{D \vec{V}}{D t}=-\vec{\nabla} p
$$

And for one-dimensional flow, 


$$
\frac{\delta u}{\delta t}+u \frac{\delta u}{\delta x}+\frac{1}{\rho} \frac{\delta p}{\delta x}=0
$$

Anderson goes on to apply the method of characteristics and Riemann invariants along with the following equation for a calorically perfect gas:

$$
a^{2}=\frac{\eta p}{\rho}
$$

Also, recalling that the process is isentropic, the following equation also applies:

$$
p=c_{1} T^{\gamma /(\gamma-1)}=c_{2} a^{2 \gamma /(\gamma-1)}
$$

Finally, the next equation relates $a$ and $u$ at any local point in a simple expansion wave:

$$
\frac{a}{a_{\infty}}=1-\frac{\gamma-1}{2}\left(\frac{u}{a_{\infty}}\right)
$$

Recalling that $a=\sqrt{\gamma R T}$ and implementing into the previous equation gives the following,

$$
\frac{T}{T_{\infty}}=\left[1-\frac{\gamma-1}{2}\left(\frac{u}{a_{\infty}}\right)\right]
$$


And because the flow is isentropic, $p / p_{\infty}=\left(\rho / \rho_{\infty}\right)^{\gamma}=\left(T / T_{\infty}\right)^{\gamma /(\gamma-1)}$. Hence, the previous equation yields the pressure and density ratio in a simple expansion wave as a function of the local gas velocity in the wave,

$$
\begin{aligned}
& \frac{\rho}{\rho_{\infty}}=\left[1-\frac{\gamma-1}{2}\left(\frac{u}{a_{\infty}}\right)\right]^{2 /(\gamma-1)} \\
& \frac{p}{p_{\infty}}=\left[1-\frac{\gamma-1}{2}\left(\frac{u}{a_{\infty}}\right)\right]^{2 \gamma /(\gamma-1)}
\end{aligned}
$$

Based on the previous assumptions by Ashley that if a piston generates only simple waves and produces no entropy changes, the exact expression for the instantaneous pressure $p(t)$ on its face is depicted by the simple unsteady wave shown here as Equation (3-16).

Lighthill [1952] states that the unsteady wave equation is expected to be reasonably accurate if the pressure on the surface remains within the range 0.2 to 3.5 times the mainstream pressure. These values are obtained with the conditions that motions have large Mach number and $M[\delta+(\varepsilon / c) \times(\omega c / U)]<1$. Lighthill assumes that $M \delta$ is bounded by less than 1 where $\delta$ is the maximum inclination of the airfoil surface to the stream or impact angle. These limitations are based on the assumption that $|u|$ in the unsteady wave equation never exceeds the speed of sound in the undisturbed 
fluid. In an oscillation with maximum displacement, $\varepsilon$ and frequency $\omega / 2 \pi$, the condition for $u$ is written by Lighthill as

$$
\varepsilon \omega+U \delta<a_{\infty}
$$

By dividing Eq. (3-17) by $a_{\infty}$,

$$
M[\delta+(\varepsilon / c) \times(\omega c / U)]<1
$$

where $M=U / a_{\infty}$ and $\mathrm{c}$ is the chord of an airfoil such that $\omega c / U$ is the frequency parameter. Finally, Lighthill discusses that the unsteady wave equation may be improved by use of the "shock expansion" theory, which takes into account the exact pressure change at the shock and assumes a simple wave behind the shock. This concept is very similar to the proposed "Piston Perturbation" method which is discussed in the next paragraph.

Since a clear limitation of the unsteady wave equation is the impact angle $\delta$, it would stand to reason based on Eq. (3-18) with $M \gg>1$ that the smaller the impact angle the better the results. This is precisely the reason why the cone in Figure 3.2 shows much better results than the same cone at the same angle in Figure 2.2. Recall that in Figure 3.2 , a $10^{\circ}$ cone is perturbed $2.5^{\circ}$ form the mean flow which in this case was determined by shock expansion theory. By making the impact angle, $\delta$, much smaller and perturbing about the mean flow, the accuracy of the surface pressure is significantly improved. 
An application of the unsteady wave equation as a small perturbation to a given mean flow in STARS is derived below. Refer to Figure 3.4.

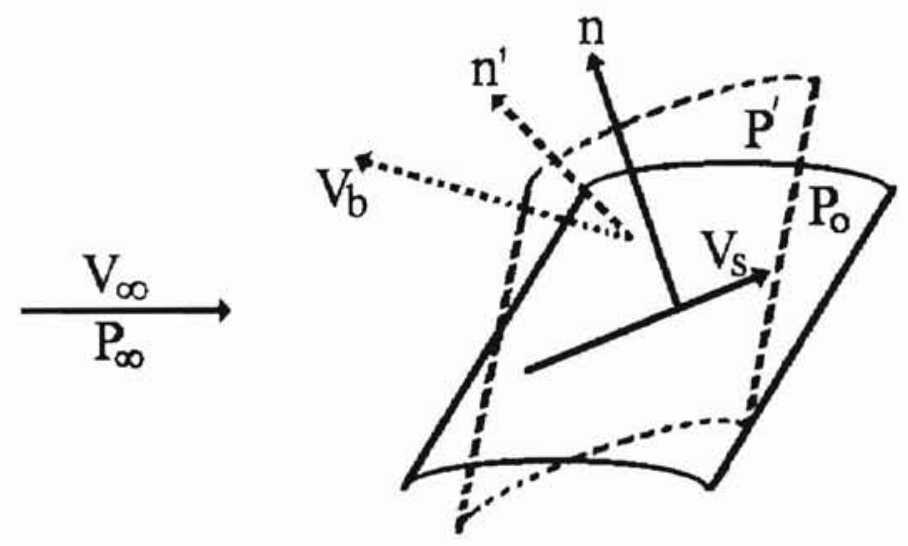

Figure 3.4. Simplistic Illustration Of A Locally Applied Perturbation To The Mean Flow

As before, the same equations are revisited. The perturbed pressure, $P^{\prime}$, is solved with respect to $P_{\infty}$ as follows,

$$
\frac{P^{\prime}}{P_{\infty}}=\frac{P_{o}}{P_{\infty}} \frac{P^{\prime}}{P_{o}}
$$

The unsteady wave equation is applied as a small perturbation to the mean flow which in this case is also $\frac{P_{o}}{P_{\infty}}$,

$$
\frac{P^{\prime}}{P_{\infty}}=\frac{P_{o}}{P_{\infty}}\left[1+\frac{\gamma-1}{2} \frac{\Delta u}{a_{o}}\right]^{\frac{2 \gamma}{\gamma-1}}
$$


aeroelastic effects. The modes of vibration are perturbed which represents a perturbation to the mean flow. Next, an application of the isentropic wave equation previously discussed is locally applied as a perturbation to the mean flow at every point. The pressure ratios obtained by the P.S. are then used in the coupled structural dynamic solutions which are numerically integrated to find a generalized displacement "q" shown as:

$$
[M] \cdot \ddot{q}+[K] \cdot q=[P]
$$

Here $[\mathrm{M}]$ and $[\mathrm{K}]$ are the mass matrix, and the stiffness matrix respectively, obtained in STARS by a finite element structural analysis routine given the structural properties of the system. $[\mathrm{P}]$ is the force matrix obtained by the piston perturbation method. Once the generalized displacement for every point on the aeroelastic surface is determined, the values are multiplied by the mode shapes to determine the actual displacement for calculation of a new set of transient data.

Given the new structural deflections based on the previous aerodynamic pressures, a new outward normal velocity is given for the next calculation of the pressures. This process repeats until enough transient data is acquired to determine the stability characteristics of the perturbed aerodynamic system. Finally, the flutter boundaries are then verified and refined with the non-linear time marching Euler method. 


\title{
CHAPTER 4
}

\section{RESULTS}

It will be shown that the Piston Perturbation method accurately predicts surface pressure for a number of different geometrical configurations in the supersonic flow regime. It will also be shown that the estimated flutter boundaries of a Generic Hypersonic Vehicle (GHV) obtained by this method are sufficiently close to the flutter boundaries obtained by the Euler method. Hence, the estimated CFD solution may then be used for refinement which requires significantly fewer transient calculations. This GHV is also used to compare predicted flutter boundaries, and run time between the perturbation solution and the Euler solution.

\author{
Simple Perturbed Wedge
}

A simple perturbed wedge exemplifies the validly of this method over a range of Mach numbers for perturbations about the mean flow in compression and expansion shown in Figure 4.1 and Figure 4.2. respectively. 


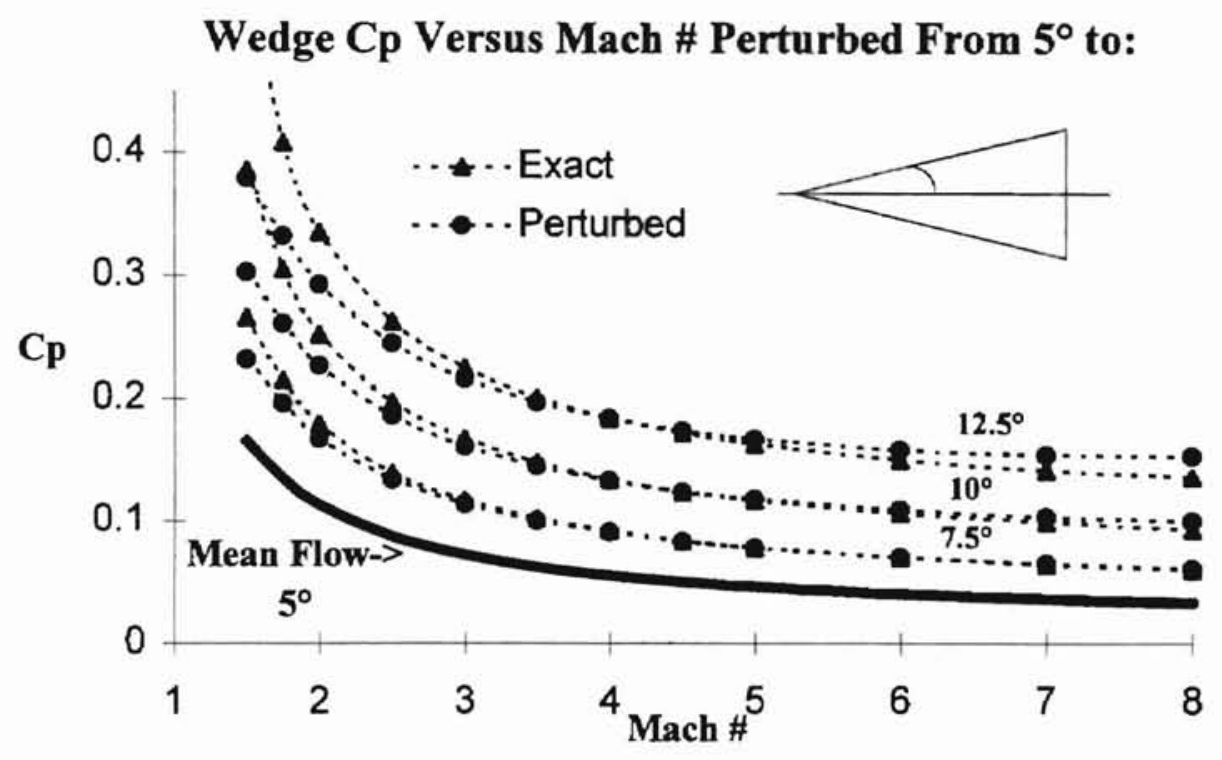

Figure 4.1. A Simple Perturbed Wedge In Compression

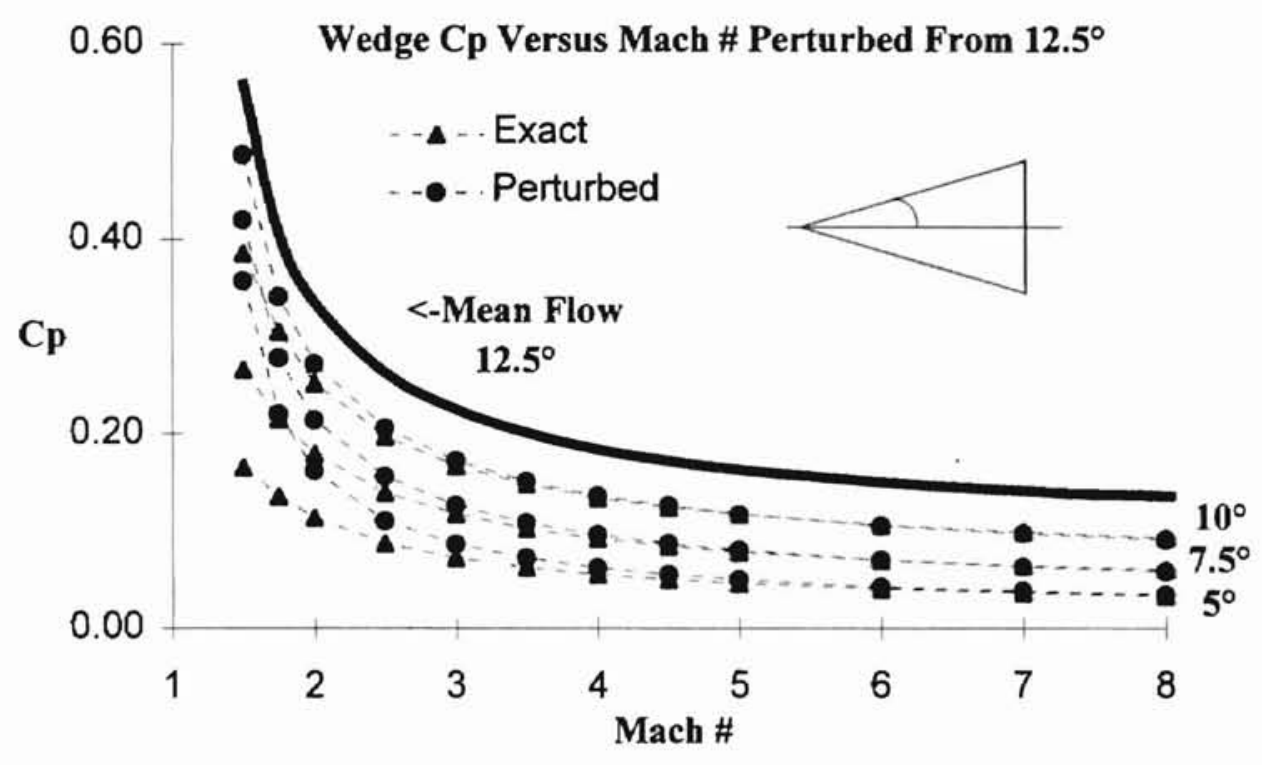

Figure 4.2. A Simple Perturbed Wedge In Expansion

The errors for this process are minimal when the assumptions and limitations previously discussed by Ashley and Lighthill are applied. These assumptions again are that the 
pressure ratios does not exceed the range of 0.2 to 3.5. with $\delta$ small and $\mathrm{M} \gg>1$, these assumptions are met. Since the majority of flutter analysis deals mostly with small perturbations this criteria holds for a wide range of Mach numbers and geometries.

To better explain the benefits and limitations of this method, a series of charts similar to Figure 4.1, and Figure 4.2 are generated. The left column of the figure is similar to the above figure and the column on the right will show the representative percent error as compared with an NACA Technical Note titled "Tables and charts of Flow Parameters Across Oblique Shocks" by Neice [1948]. The first set of charts shown below in Figure 4.3 illustrate a more complete set of data for a simple wedge in compression. Notice how the percent error increases as the angle from which the solution is perturbed decreases. This shows that a small perturbation about a strong mean flow condition gives more accurate results. Also note how the percent error increases proportional to the perturbation angle. This phenomena shows that by increasing $\delta$, the piston velocity starts to approach the local speed of sound which is an obvious limitation. Next, a similar series of charts show a more complete data set for a simple wedge in expansion.
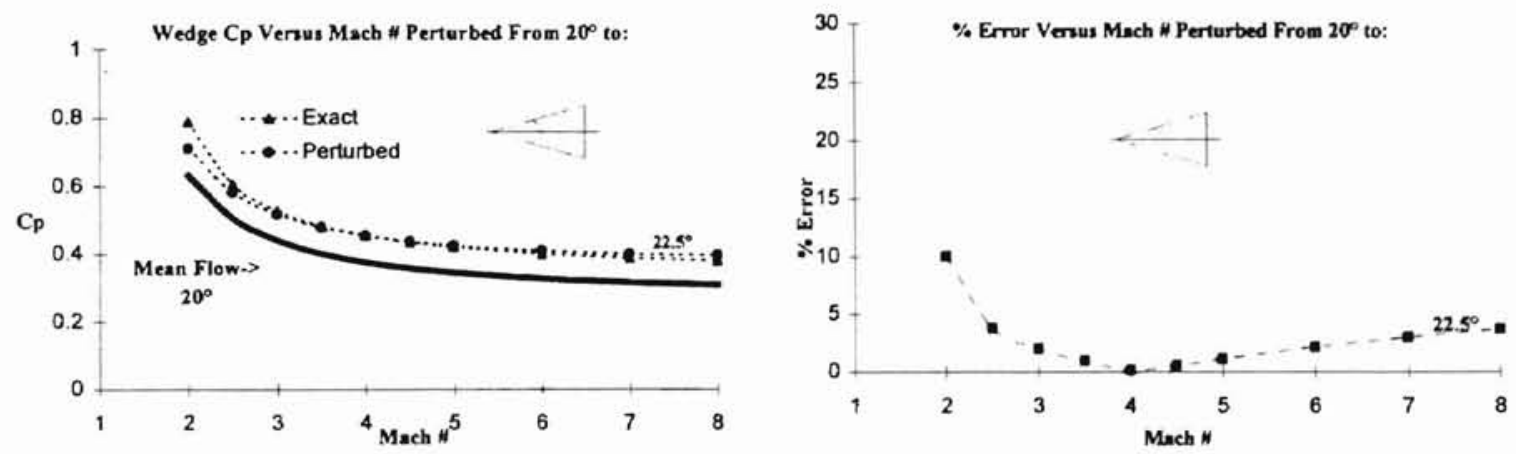

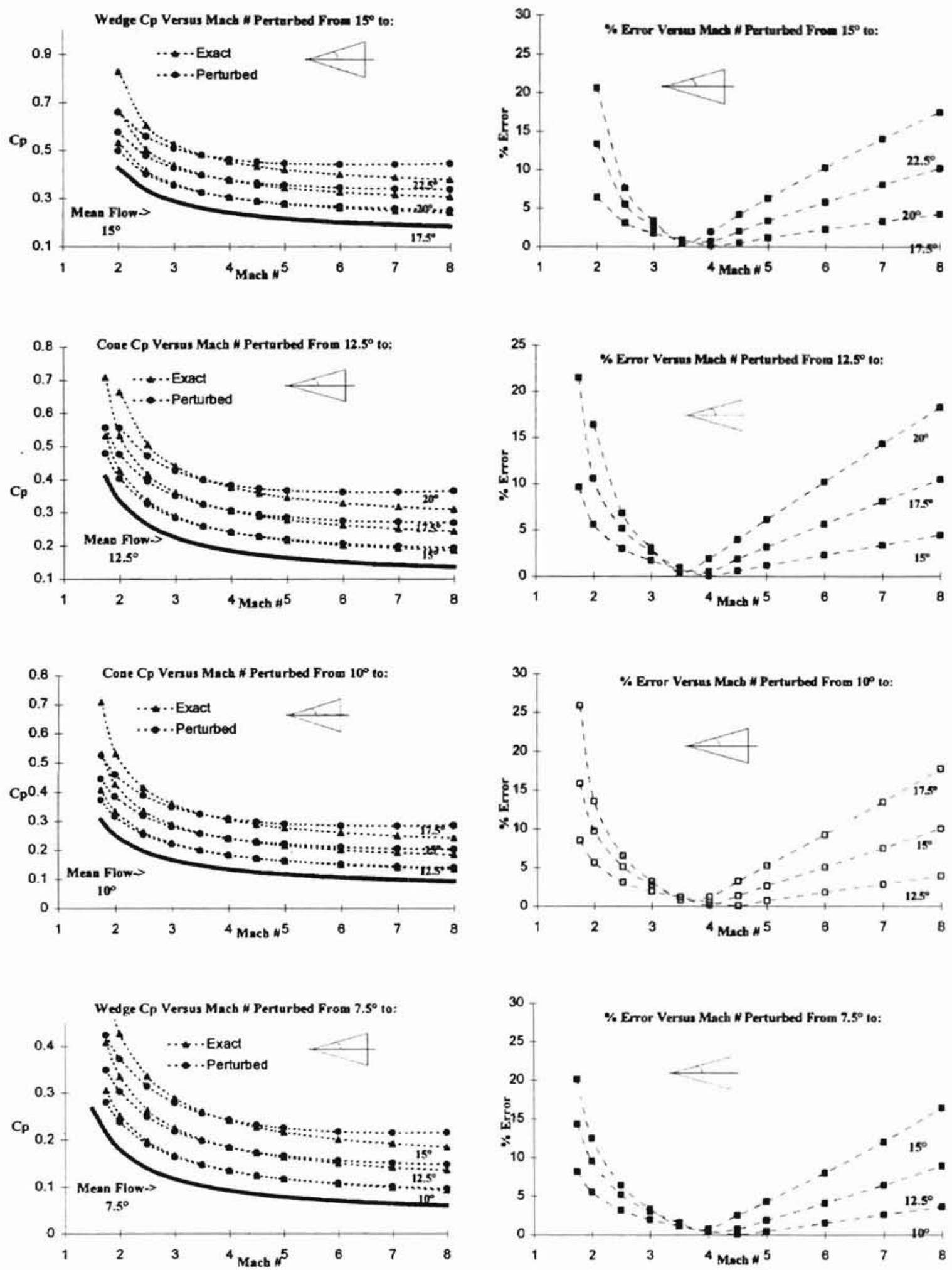

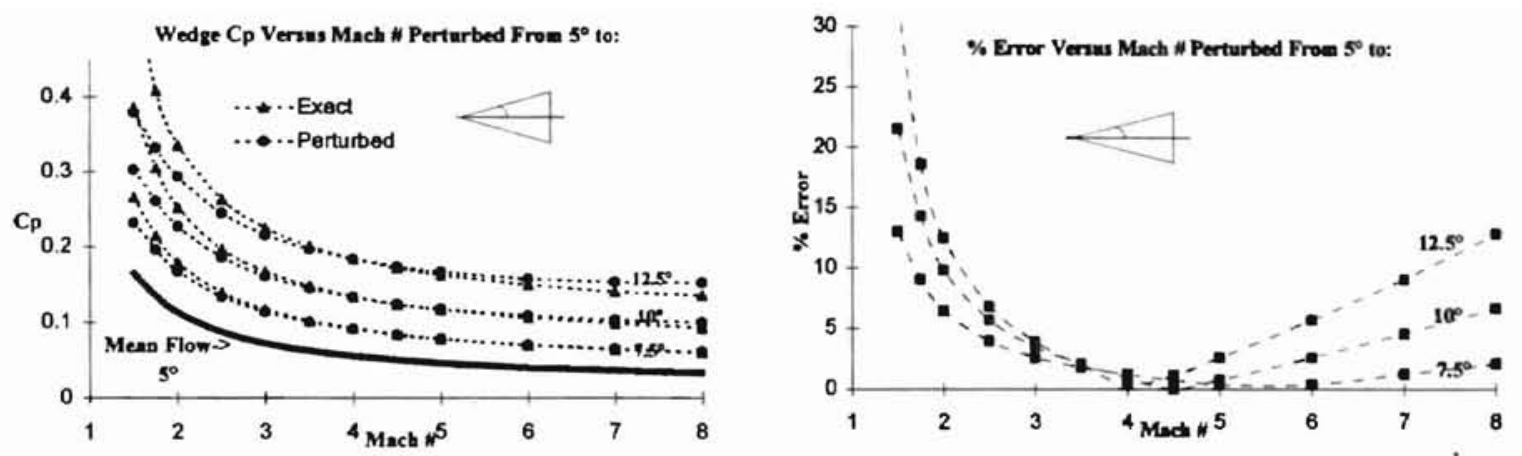

Figure 4.3. More Complete Data Set For A Simple Wedge In Compression
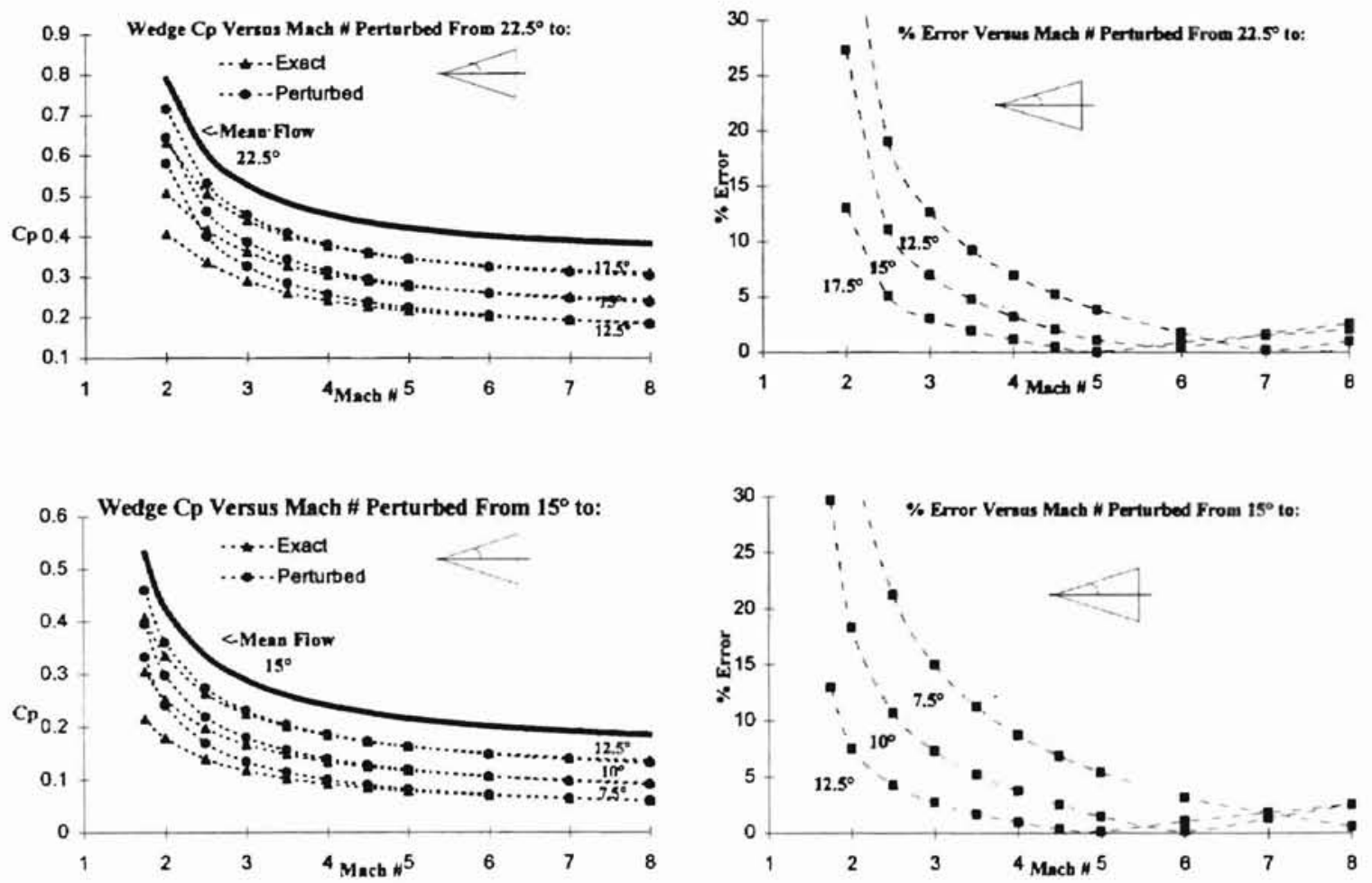

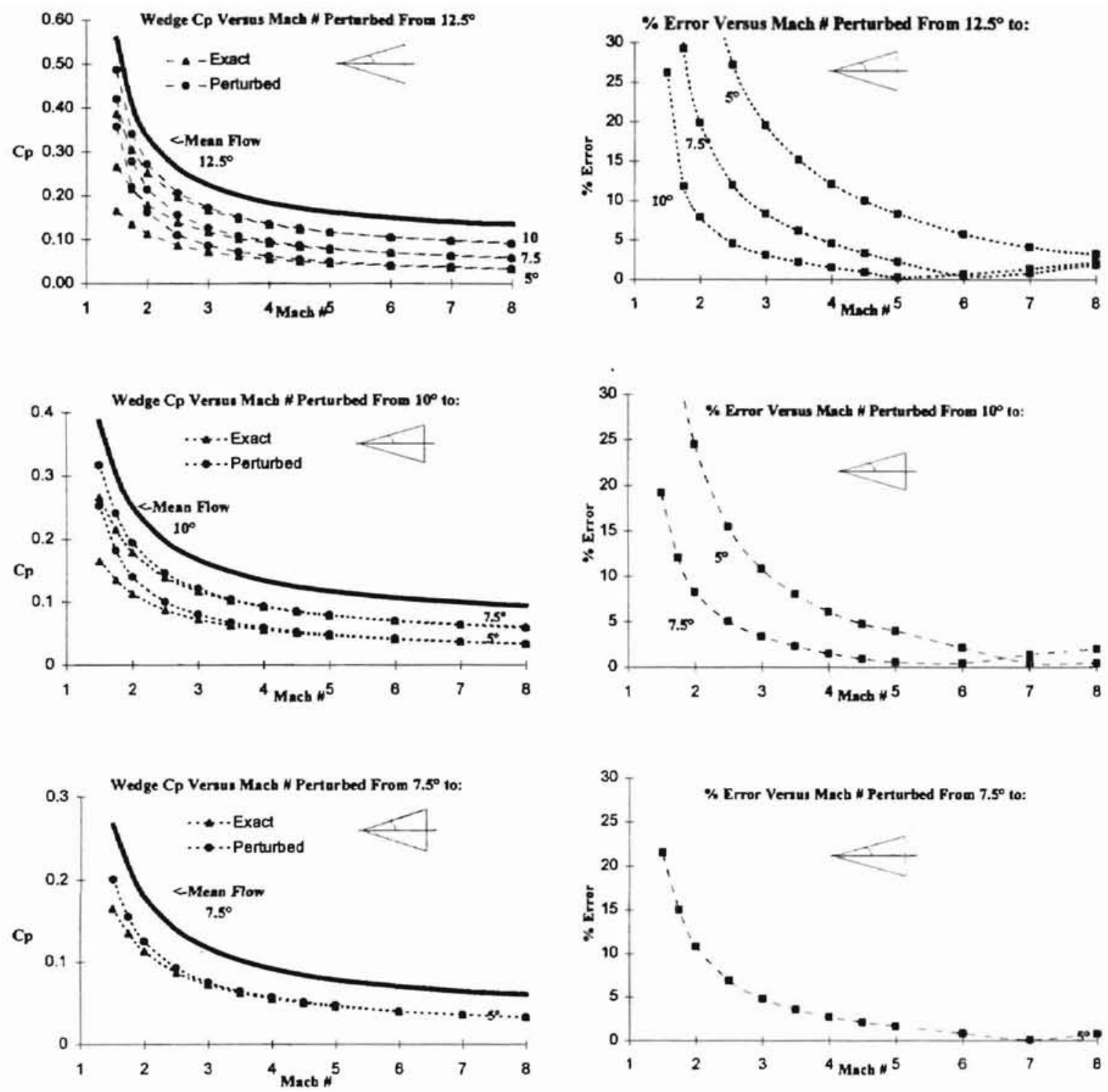

Figure 4.4. A More Complete Data Set For A Simple Wedge In Expansion 


\section{Simple Perturbed Cone}

The P.S. also predicts pressure about more three dimensional surfaces, such as cones, over a range of Mach numbers in compression as well as expansion. This is shown in Figure 4.5 and Figure 4.6. Again, the P.S gives accurate results of surface pressure since it is applied as a small perturbation to the mean flow where the threedimensional relaxation effects are accounted for.

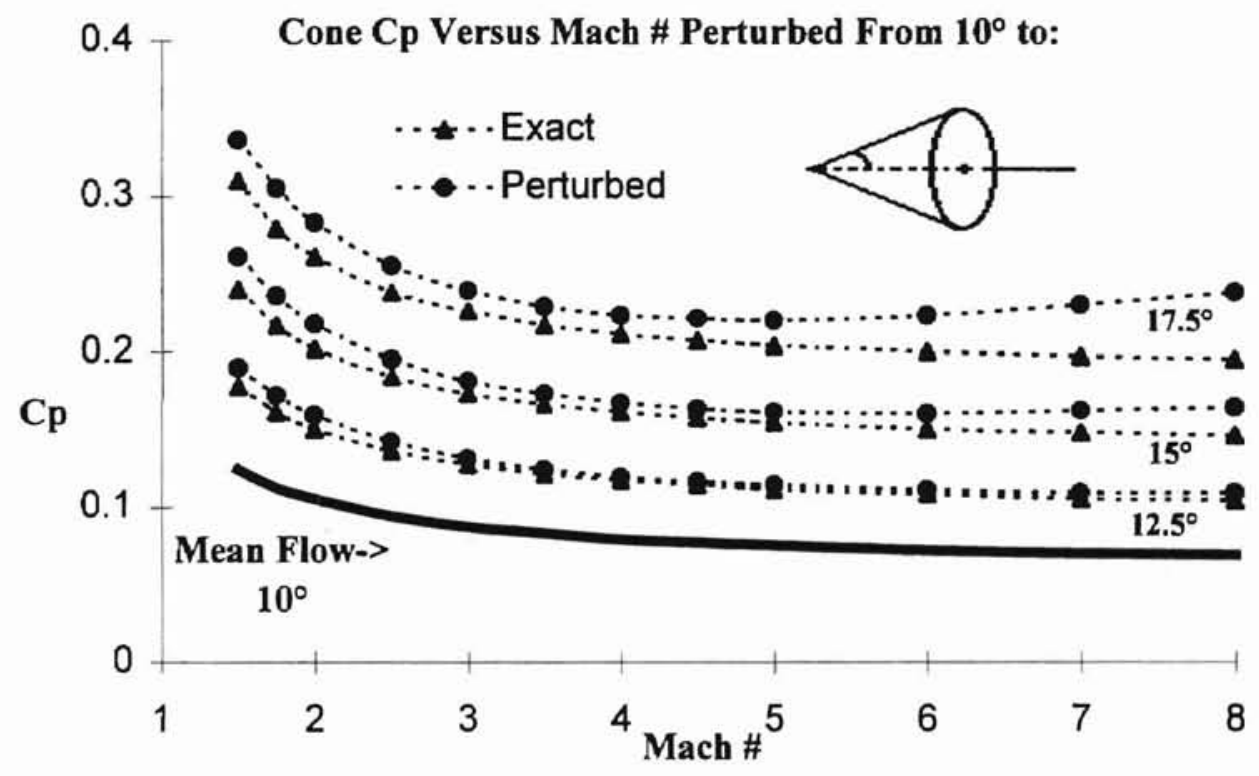

Figure 4.5. A Simple Perturbed Cone In Compression 


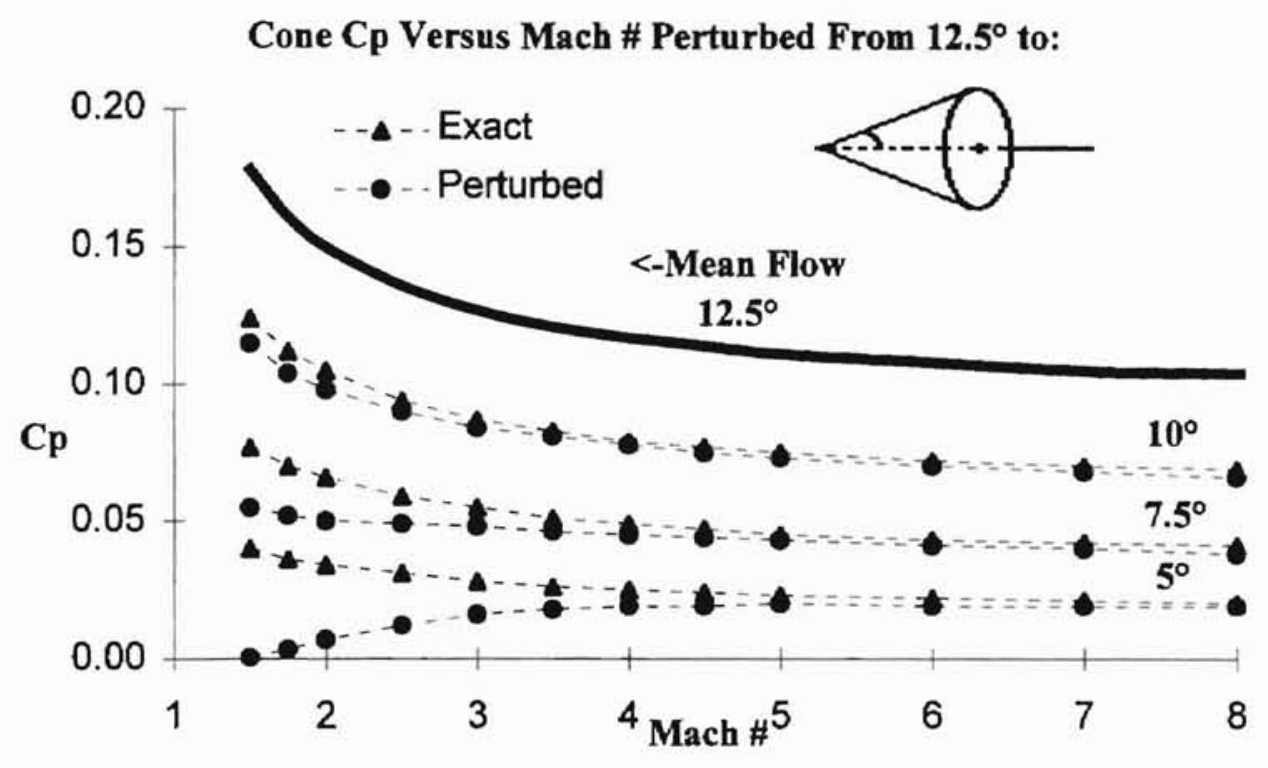

Figure 4.6. A Simple Perturbed Cone In Expansion

Notice the perturbed curve from $12.5^{\circ}$ to $5^{\circ}$ shown in Figure 4.6 above. This is the result of a perturbation condition greater than the mean flow condition. Hence, an obvious limitation of this method. Additional illustrations of cone perturbation similar to the previous two figures are shown below. Since these charts show perturbations of cones with various angles, this phenomenon will be shown again in expansion.
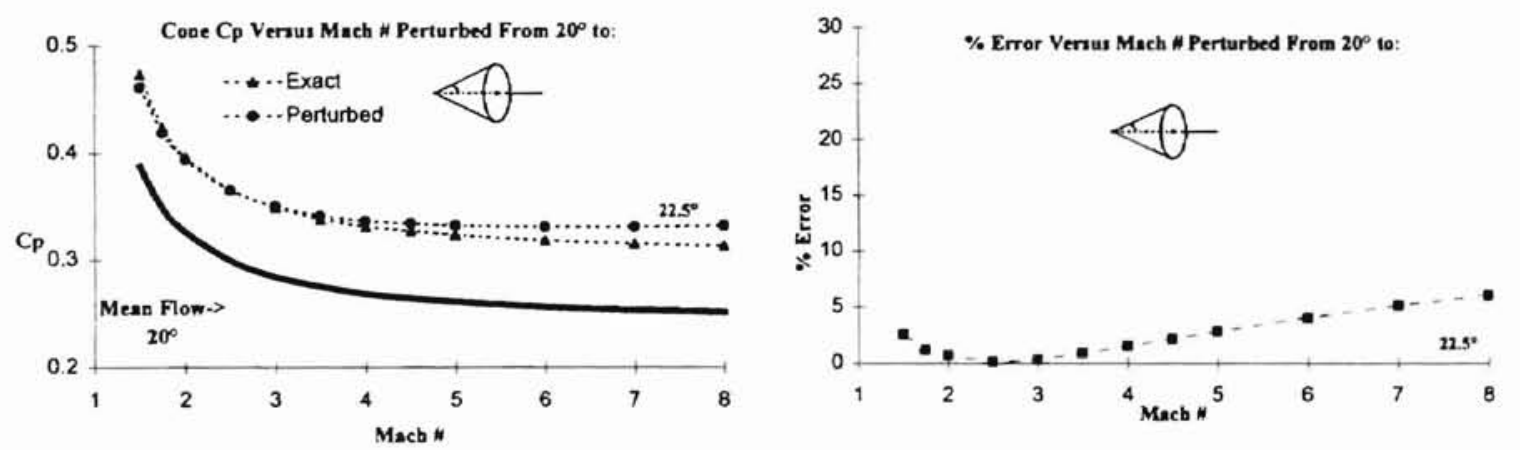

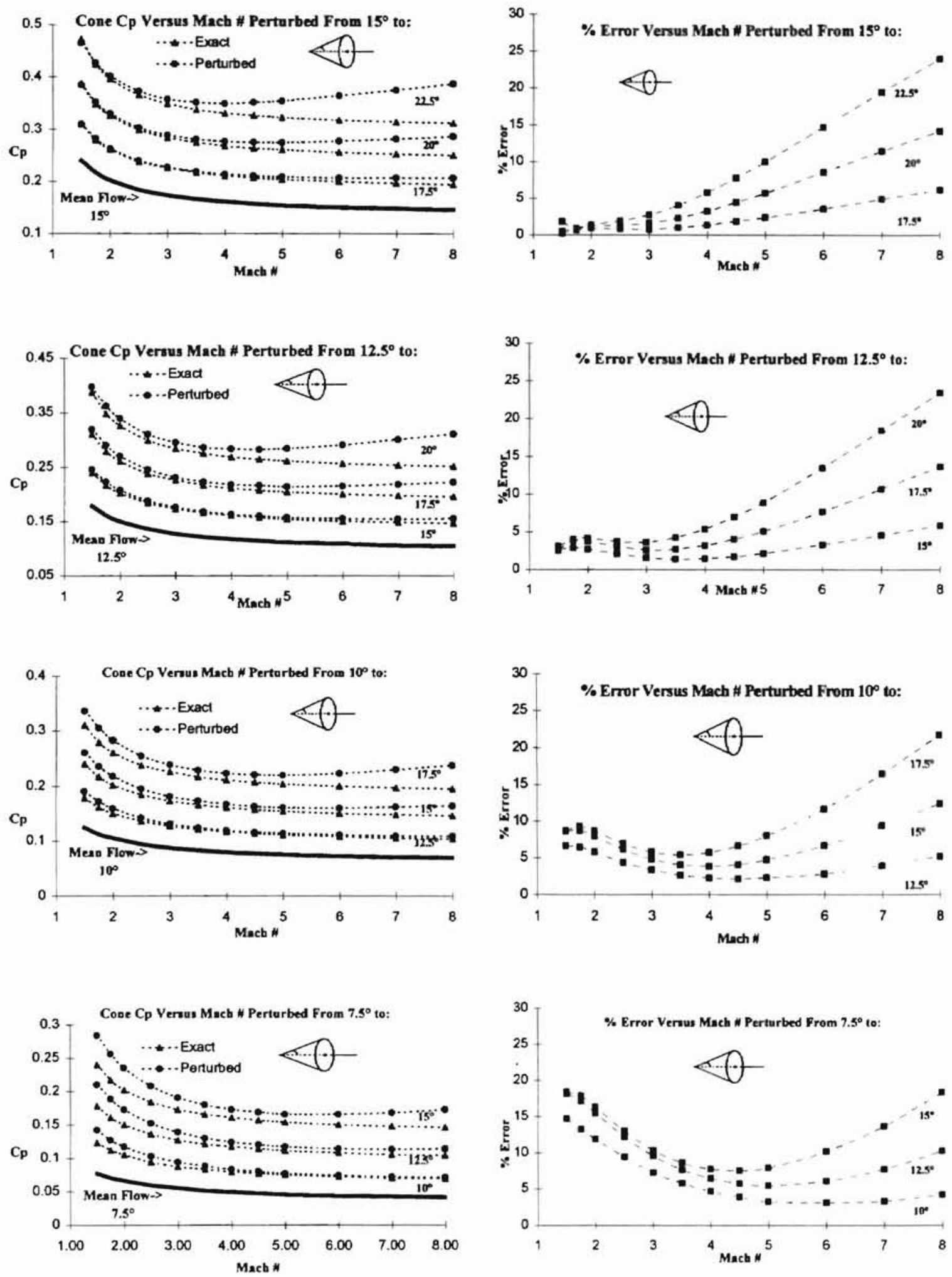

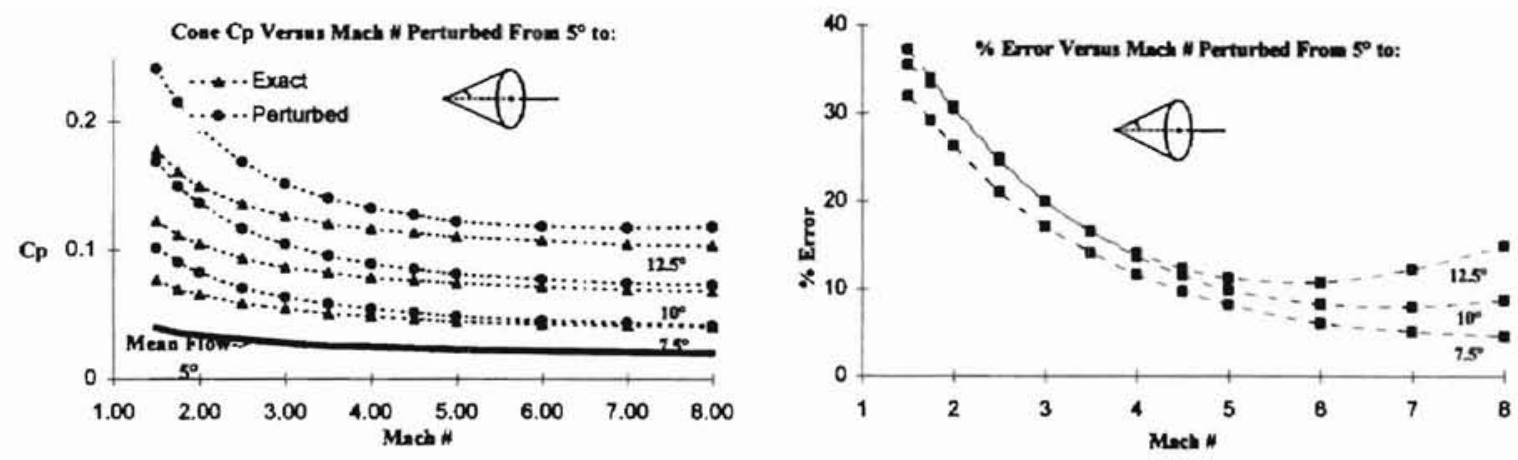

Figure 4.7. A More Complete Data Set For A Simple Cone In Compression
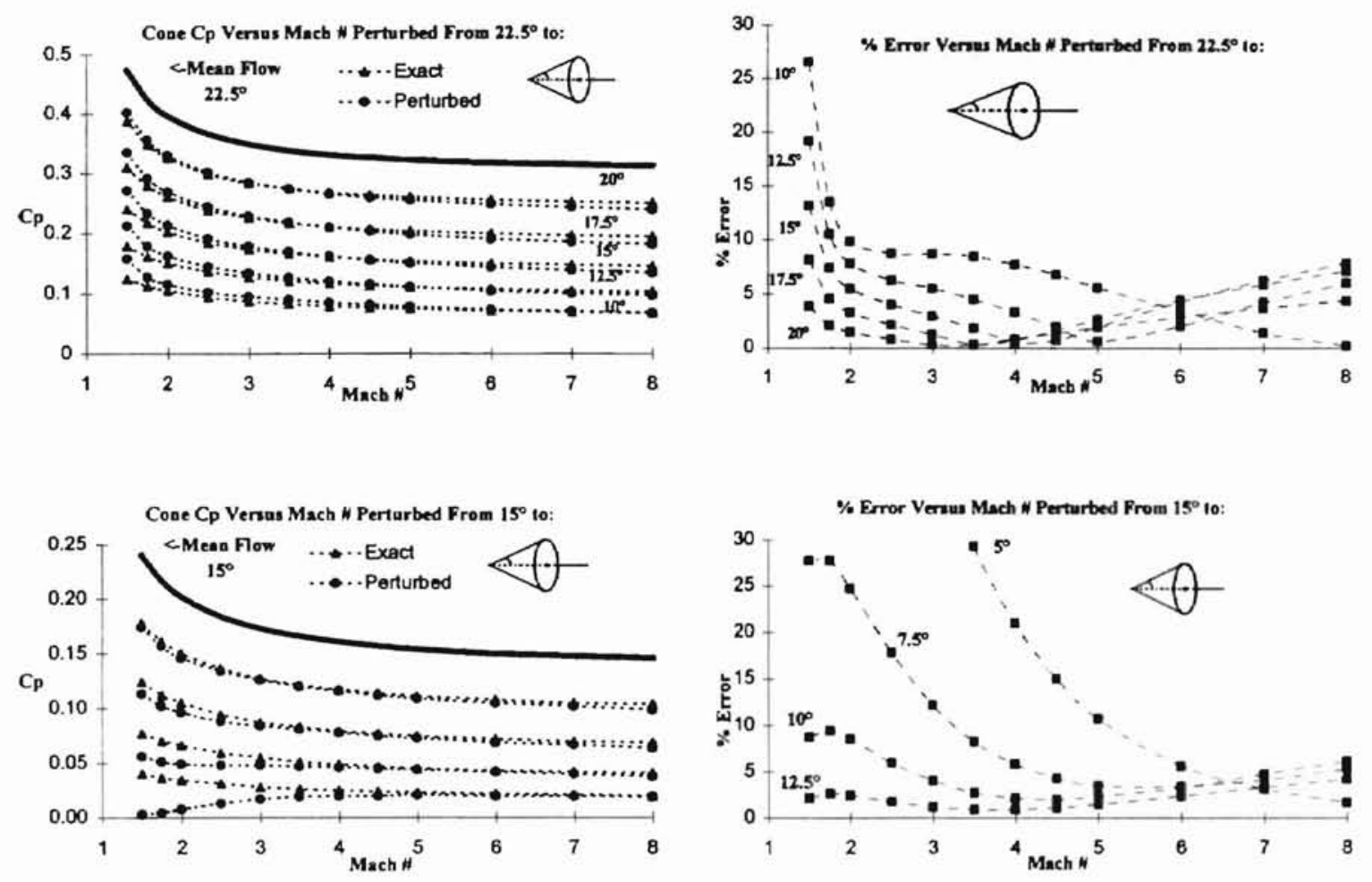

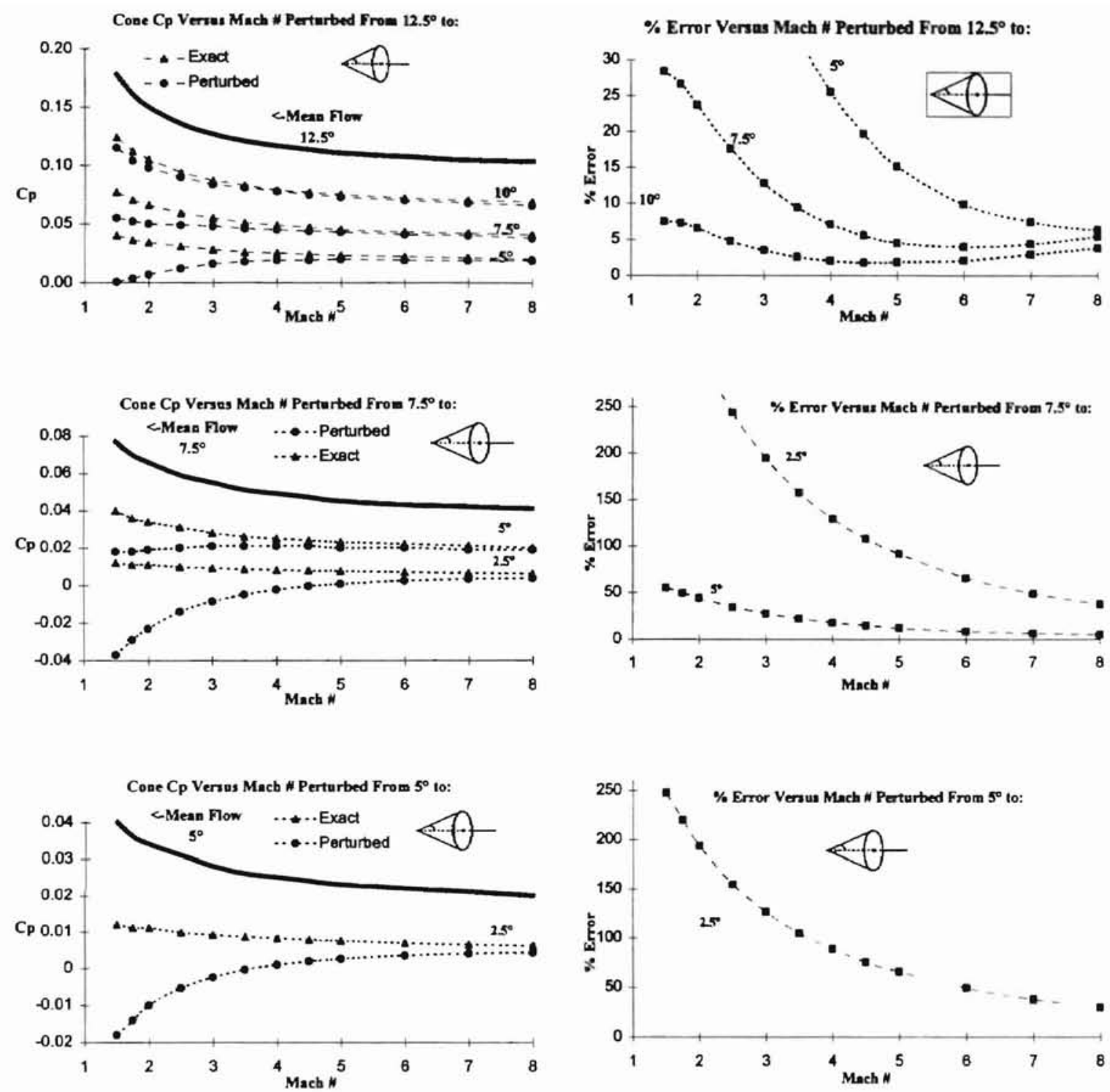

Figure 4.8. A More Complete Data Set For A Simple Cone In Expansion

\section{Clamped Plate With Heavy Shock Interaction}

Another advantage of the Perturbation method is the ability to capture nonlinearities such as shock interactions by perturbing about the mean flow where such 
effects are taken into account. As shown in the following image (Figure 4.9), at $\mathrm{M}_{\infty}=$ 2.2 , a shock induced by a rigid wedge in a fixed rectangular duct interacts with an elastic clamped flat plate with differential pressure on the outer part of the duct.

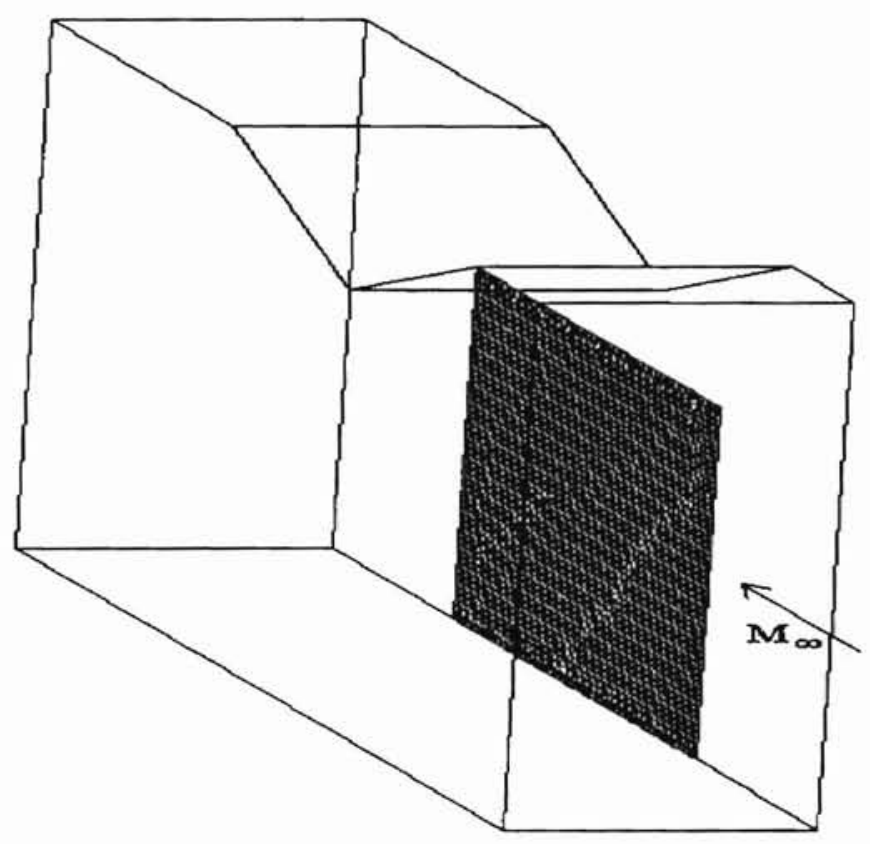

Figure 4.9. A Fixed Rectangular Duct With An Elastically Flexible Clamped Flat Plate

A side view of this geometry (Figure 4.10) shows the pressure contours generated by steady Euler analysis. Notice the shock interactions due to the wedge and rigid boundaries of the geometry. 


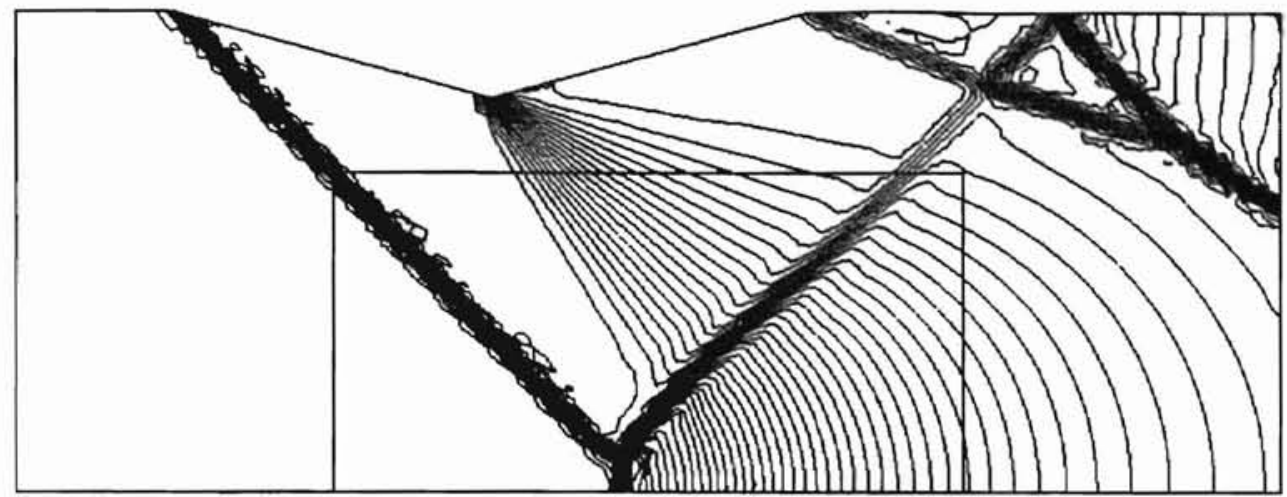

Figure 4.10. A Side View Showing The Pressure Contours Of The Heavy Shock Interactions With The Elastic Plate

A magnified view for the steady state deformation of the elastic plate generated by the unsteady Euler equations is shown in Figure 4.11(a). This deformation is generated by first running a steady Euler analysis to obtain convergence of the aerodynamic properties throughout the duct. Next, the process is restarted, this time allowing the plate to oscillate due to the resulting aerodynamic forces just acquired from the steady Euler analysis. After the plate's oscillation damps, steady state deflection is achieved. Notice the plate's outward deformation due to the shock induced by the rigid wedge.

As a comparison, unsteady aerodynamic analysis via piston theory (as opposed to unsteady Euler analysis) is performed and shown below in Figure 4.11(b). Since the piston theory takes into account only the local conditions, pressure induced by the shock is unaccounted for which causes large errors in the steady state deformation.

The Perturbation solution is now applied with the following results in Figure 4.11(c). Since the perturbation method perturbs about the mean flow, the non-linearities induced by the shock are accounted for giving more accurate results as compared with the unsteady Euler analysis shown in Figure 4.11(a). 


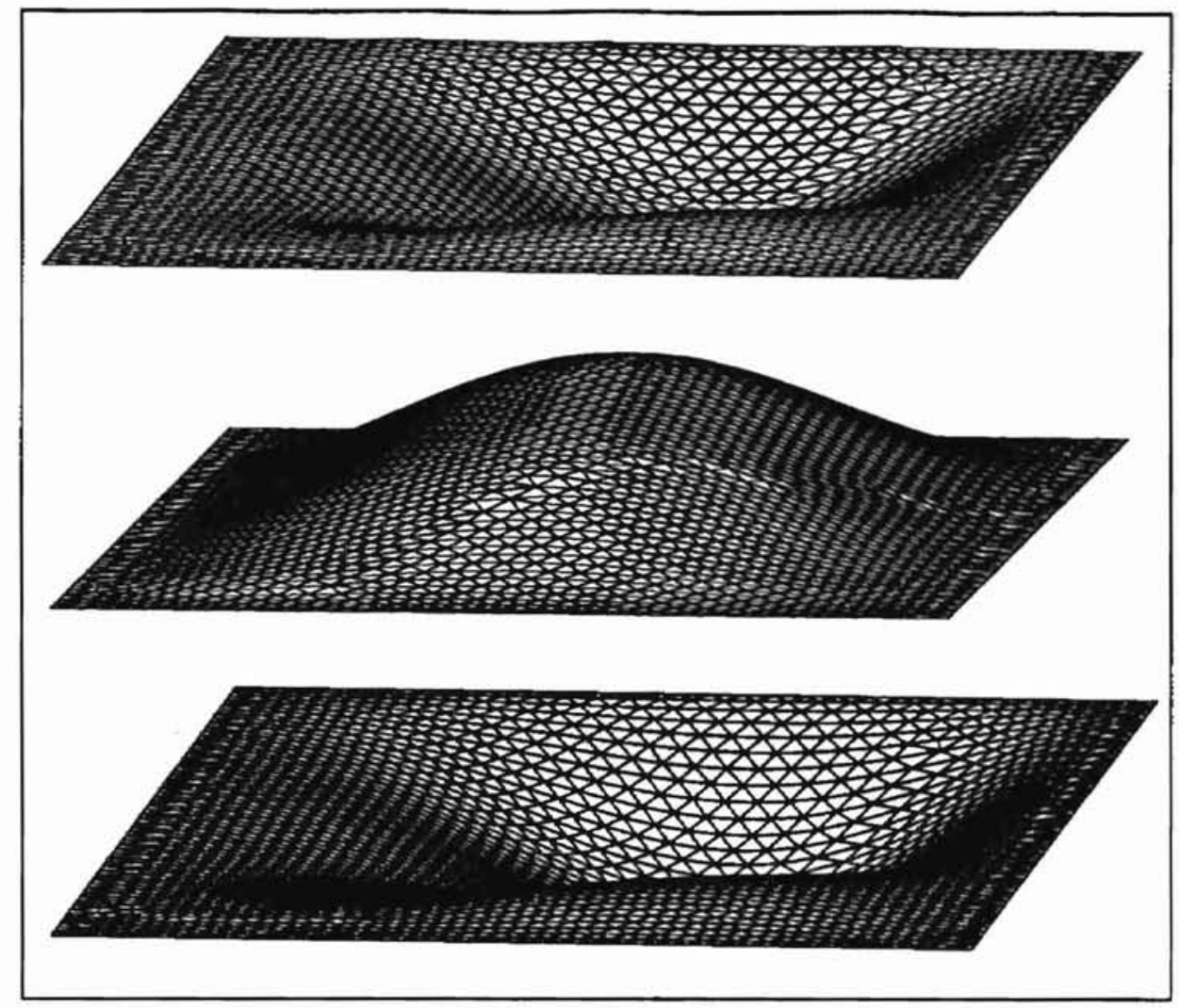

Figure 4.11. Steady State Deformation Of The Elastic Plate Generated By: (A) Unsteady Euler Analysis, (B) Piston Theory, (C) Perturbation Method

Steady Perturbation Analysis

As another example of the accuracy and generality of the Perturbation method, pressures at three sectional cuts on a Generic Hypersonic Vehicle (GHV) are calculated at Mach 2.2 with a $5^{\circ}$ angle of attack. The Perturbation method results are calculated by applying an application of the unsteady wave equation as a $1^{\circ}$ perturbation about the mean flow (calculated by steady Euler analysis) at $4^{\circ}$. . The pressure via Piston theory, and Euler analysis at $5^{\circ}$ angle of attack is also calculated for comparison and shown in Figure 4.12. 

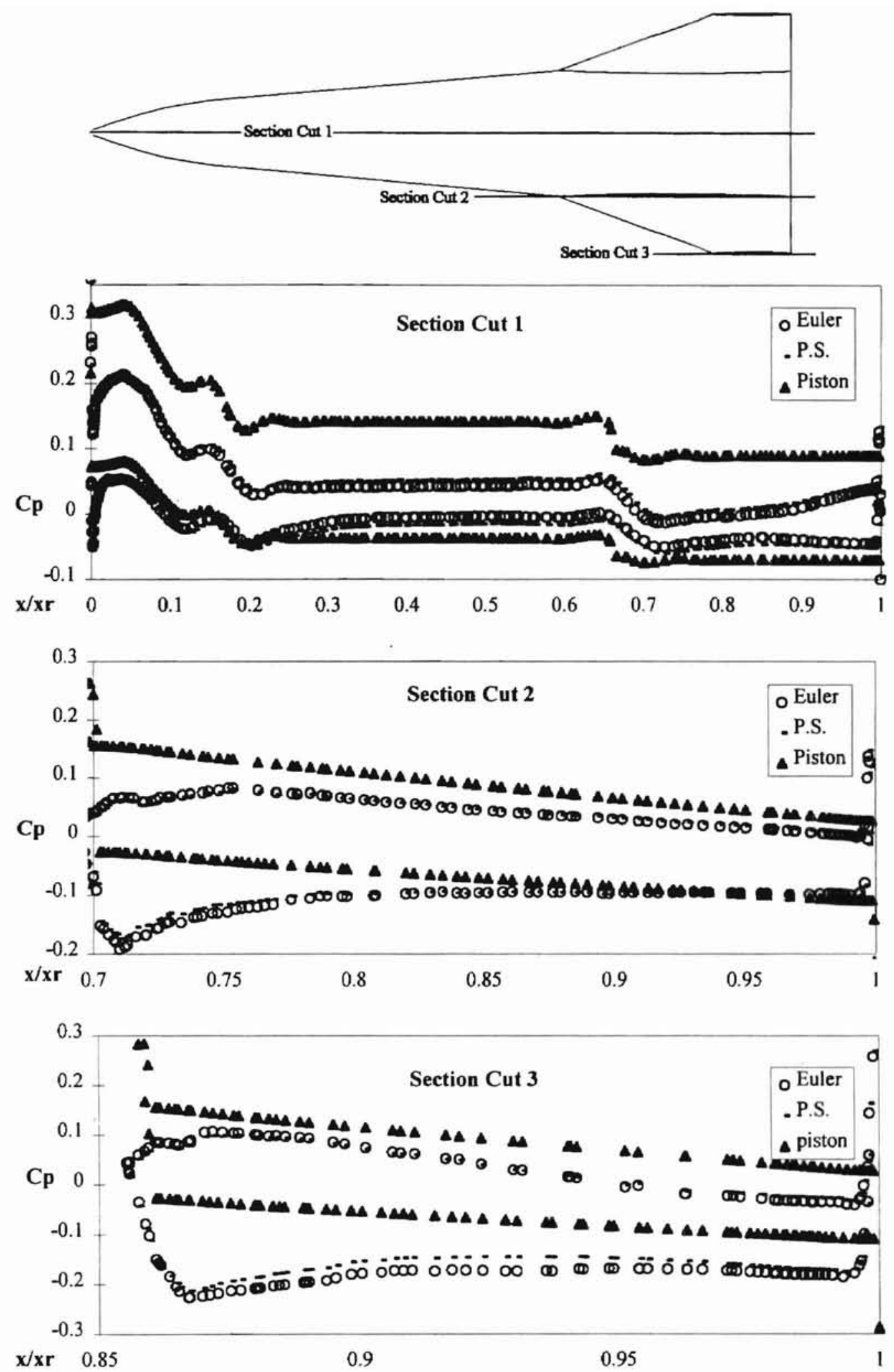

Figure 4.12. Pressure Comparisons At $5^{\circ}$ Angle Of Attack Using Euler, Piston, And Perturbation Method At Three Sectional Cuts On A GHV 
GHV Flutter Analysis

As a another illustration, a surface mesh of the baseline configuration for the GHV is generated (Figure 4.13) and flutter analysis is performed in the following manner:

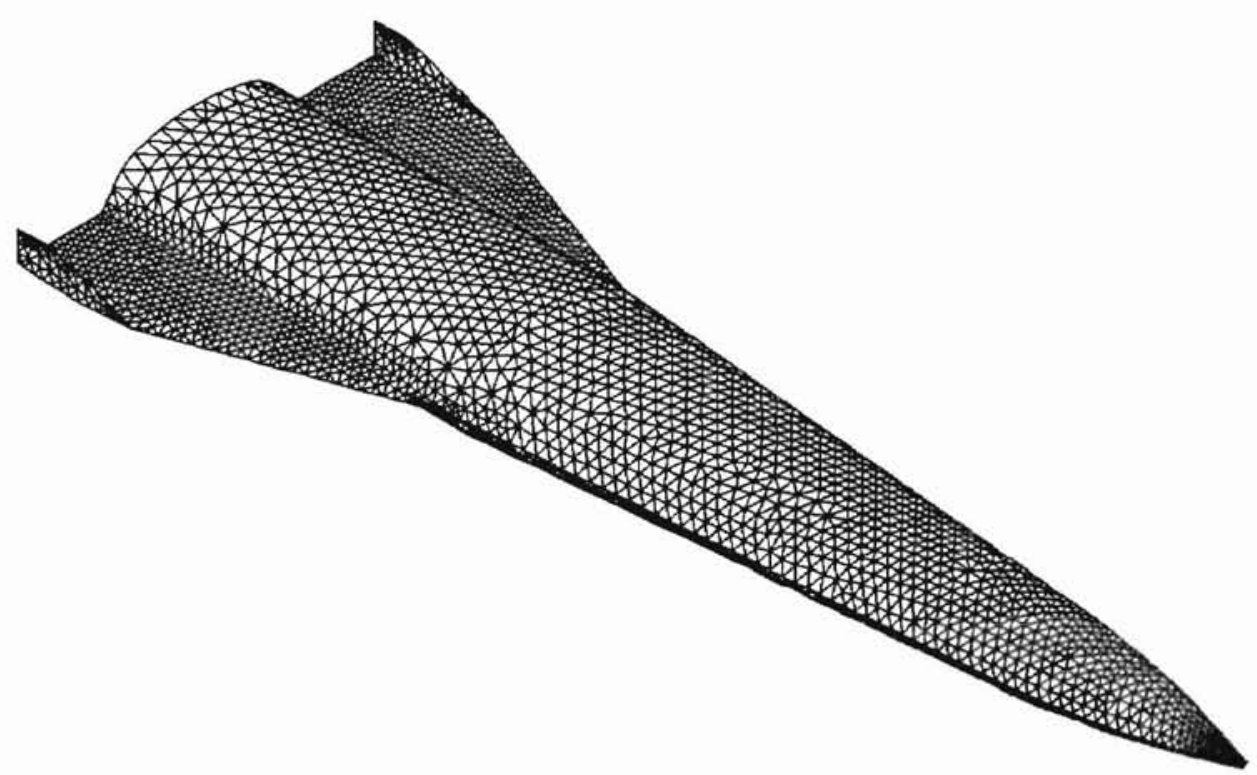

Figure 4.13. GHV Baseline Surface Mesh

Given the GHV's surface mesh, a finite-element structural model is developed to obtain the structural mode shapes and frequencies (details referenced under Gupta, 1990, 1991, 1992). Next, a steady solution to the flow field at Mach 2.2 and $0^{\circ}$ angle of attack is obtained by the finite-element Euler methodology. This solution is then used as the mean flow condition about which the perturbation solution is applied. For the aeroelastic simulation, a 9 mode solution is run for 705 time steps which is approximately 7 cycles of mode 1. Using the unsteady Euler analysis, transient data of 4 dynamic pressures is 
analyzed and the flutter boundary is estimated through polynomial interpolation. For the purpose of run-time comparison, the same procedure is used for the perturbation solution. In this case of course, the piston perturbation method is applied in place of the unsteady Euler analysis.

As seen in Figure 4.14, the difference among flutter boundary estimates between the two codes for this case is found to be less than $4 \%$, however the difference in run times between the two codes is extremely significant.
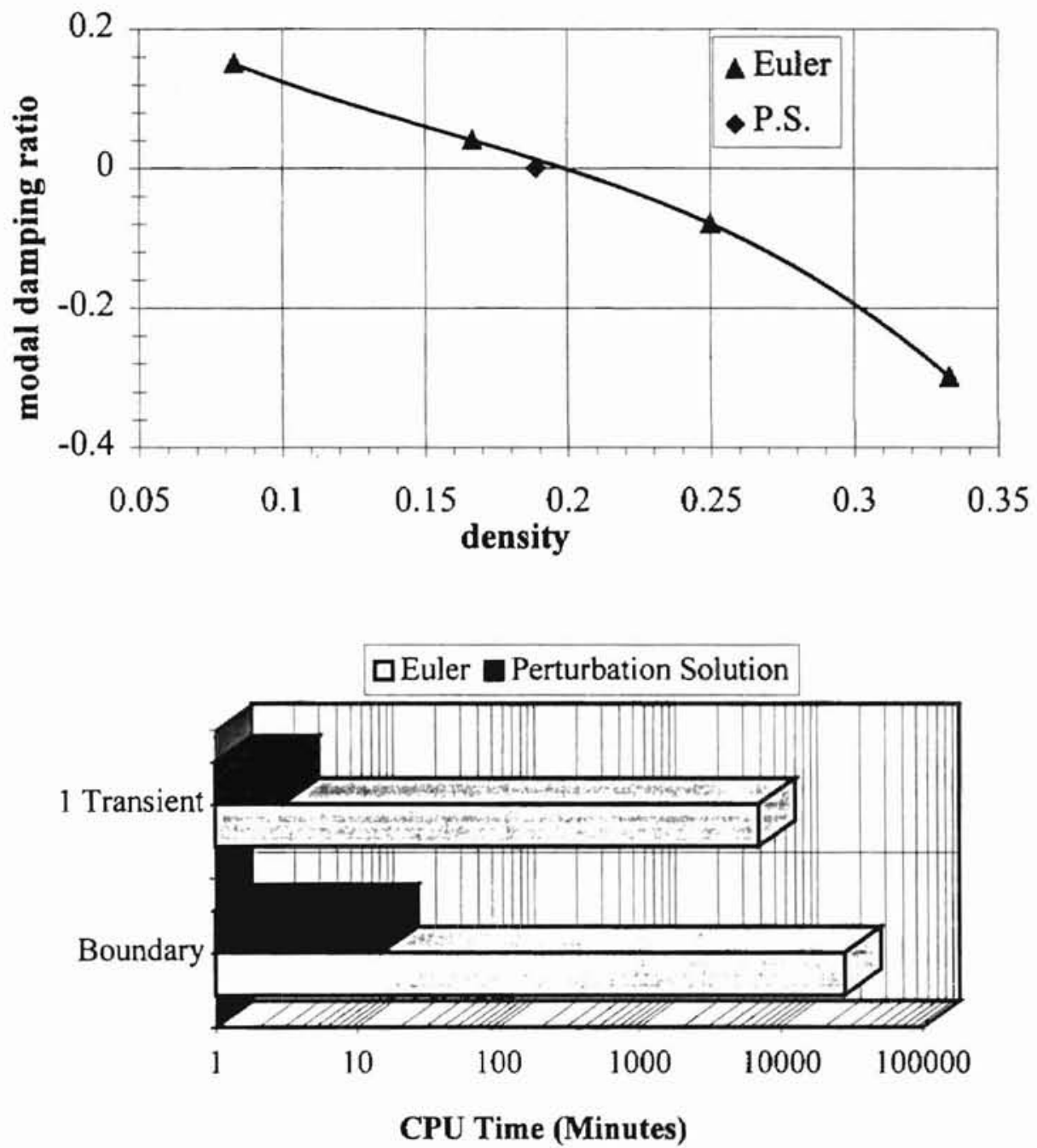

Figure 4.14. Flutter Boundary And Run-Time Comparisons At M=2.2 With 705 Time Steps / Transient 
Run time for the perturbation solution (P.S.) estimate is 3 minutes for each transient or approximately 15 minutes to identify the flutter boundary. On the other hand, run time for the Euler solution used to define the flutter boundary is 117 hours for each transient and approximately 469 hours to identify the boundary. All simulations are run on an IBM RS6000 3BT workstation.

Cone \& Swept Wing Configuration

As a final illustration of the perturbation method, a geometry consisting of a combination "Cone and Swept Wing Configuration" (CSWC) is considered to examine the flow characteristics about a more three dimensional flow field. The analysis will be done in the same manner as the GHV, with a wider test range. The following geometry (Figure 4.15) shows the baseline surface mesh used by the flow solver in the aerodynamic analysis. The symmetrically cambered wing is swept 42 degrees which makes the design Mach number when placed on the $15^{\circ}$ cone approximately 1.6 . Since steep gradients require a fine mesh and more computation time, the cone is tapered aft of the swept wing. Since the taper is gradual and the flow is supersonic (flow effects may not propagate forward), this area may be coarsely meshed aiding in computational efficiency. Figure 4.16 shows the specific dimensions of the CSWC geometry as well as detailed information used to create the aerodynamic mesh so the CSWC may be regenerated at another time. 


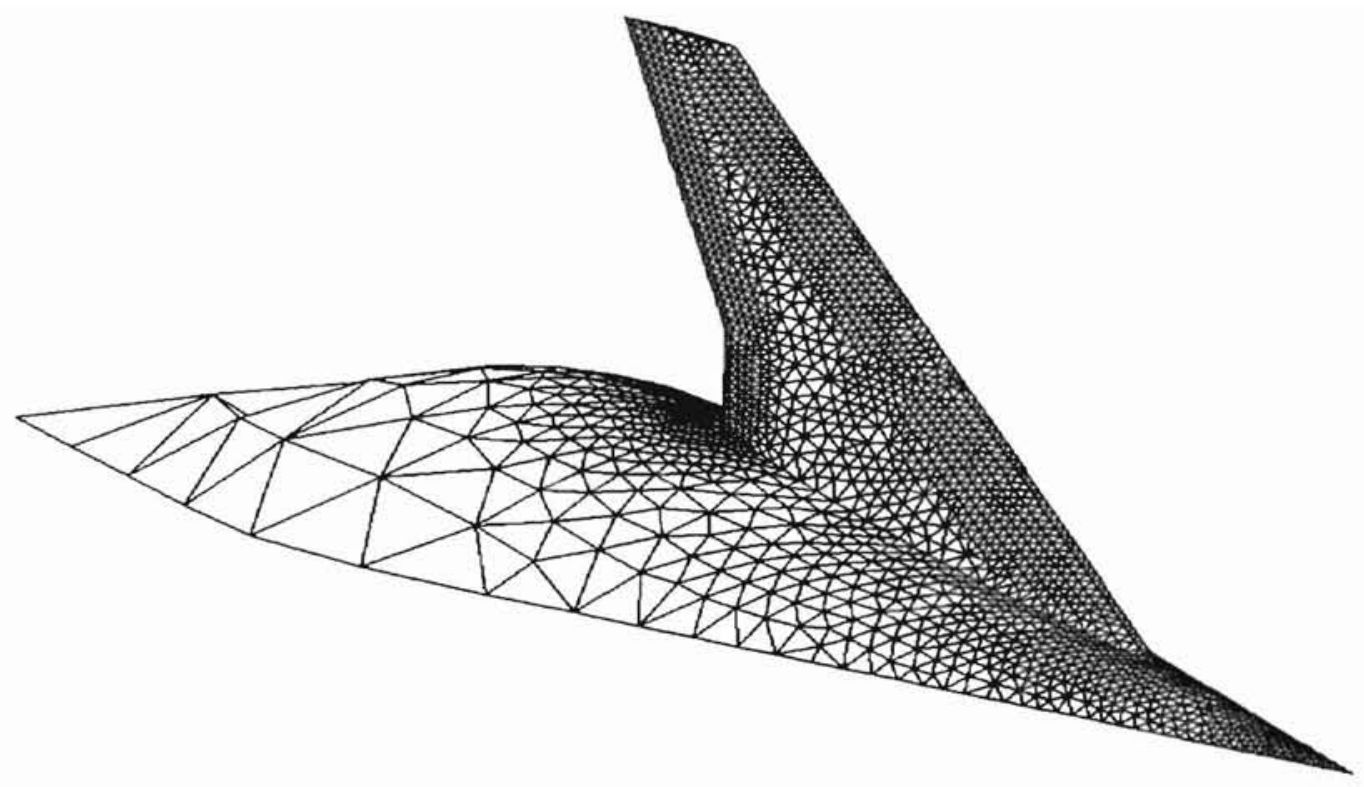

Figure 4.15. Cone \& Swept Wing Configuration Baseline Mesh

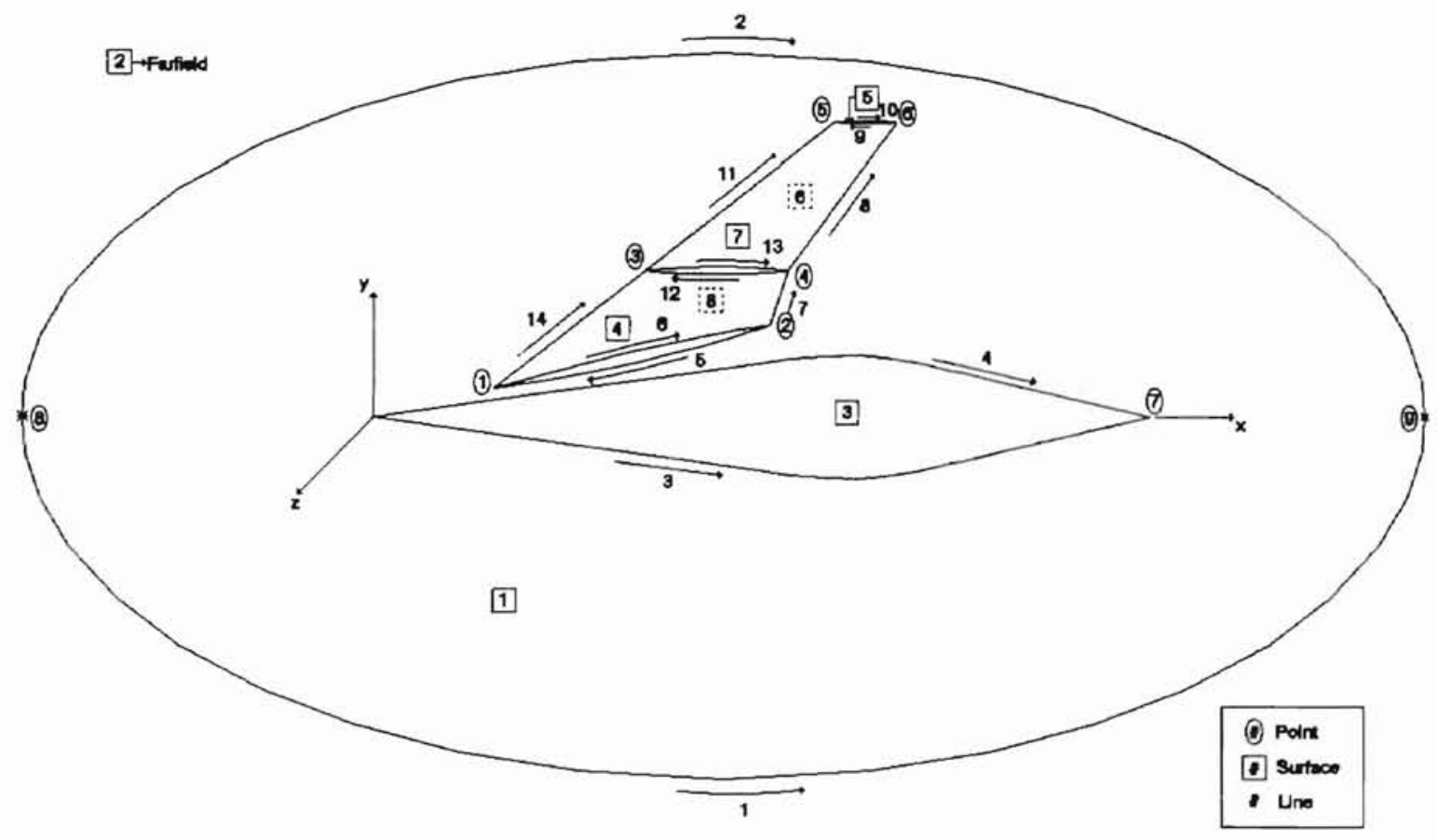

Figure 4.16. CSWC Specific Dimensions 
The values for Figure 4.16 are tabulated below in Table 4.1. Also, the data file containing further information used to generate the mesh of the aerodynamic flow solver is located in Appendix B on page 80.

Table 4.1. Point Location for The CSWC

\begin{tabular}{|c|c|c|c|}
\hline \multicolumn{4}{|c|}{ Point Location } \\
\hline$\#$ & $\mathrm{x}$ & $\mathrm{y}$ & $\mathrm{z}$ \\
\hline 0 & 0.0000 & 0.0000 & 0.0000 \\
1 & 1.7159 & 0.4598 & 0.0000 \\
2 & 5.6818 & 1.5226 & 0.0000 \\
3 & 3.8975 & 2.4242 & 0.0000 \\
4 & 5.9394 & 2.4242 & 0.0000 \\
5 & 6.6024 & 4.8597 & 0.0000 \\
6 & 7.5017 & 4.8597 & 0.0000 \\
7 & 15.0000 & 0.0000 & 0.0000 \\
8 & -5.0000 & 0.0000 & 0.0000 \\
9 & 15.0000 & 0.0000 & 0.0000 \\
\hline
\end{tabular}

The symmetrical swept wing configuration is generated using the following equation:

$$
z_{u, l}= \pm 4 \varepsilon \frac{x}{c}(c-x)
$$

$\varepsilon$ is chosen to be 0.05 which will cause the mean flow to see an impact angle of approximately $5^{\circ}$. A cross-section of this wing is shown below in Figure 4.17. 


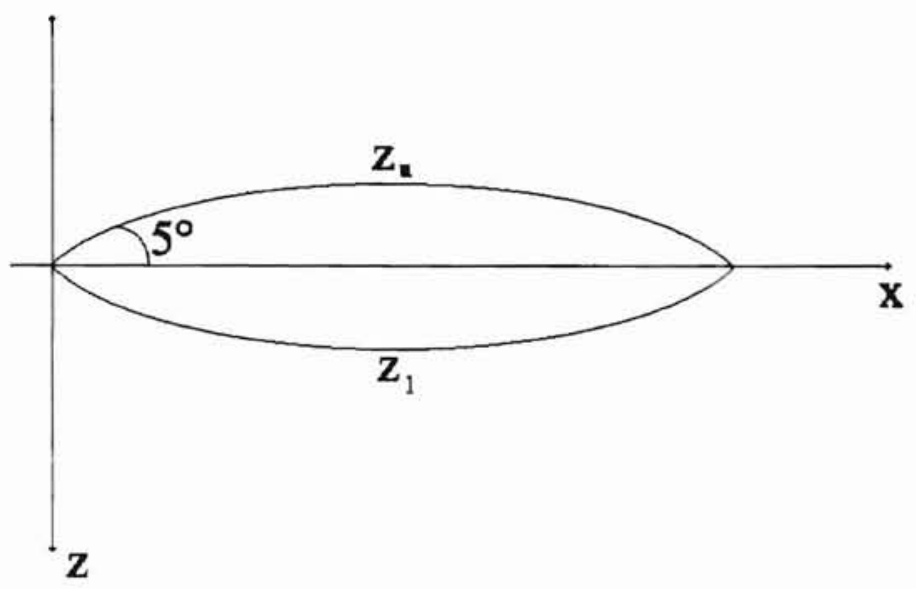

Figure 4.17. Symmetrical Wing Cross Section

The modal frequencies and material properties for the CSWC are tabulated below. Keep in mind that the only part of the structure which is aloud to deform is the wing.

Table 4.2. CSWC Structural Modal Frequencies

\begin{tabular}{|c|c|}
\hline \multicolumn{2}{|c|}{ Modal Frequencies } \\
\hline Mode & Freq. (Hz.) \\
\hline 1 & 2.9589 \\
2 & 12.1243 \\
3 & 21.9318 \\
4 & 29.2419 \\
5 & 44.1518 \\
6 & 59.1715 \\
\hline
\end{tabular}

Table 4.3. CSWC Material Properties

\begin{tabular}{|c|}
\hline Material Properties \\
\hline Poisson's Ratio $=0.33$ \\
Elastic Modulus $=71.7055 \mathrm{E} 9 \mathrm{~Pa}$ \\
Mass Density $=2783.0456 \mathrm{~kg} / \mathrm{m}^{\wedge} 3$ \\
Thickness $=0.04 \mathrm{~m}$
\end{tabular}


Flutter analysis at Mach 1.3 is one Mach number considered along with Mach 1.6, Mach 2.0, Mach 2.4, and Mach 2.8. Figure 4.18 shows the pressure contours generated by the steady finite element Euler analysis at Mach 1.3. The pressure contours shown here are used as the mean flow condition for the unsteady Euler analysis and the P.S.

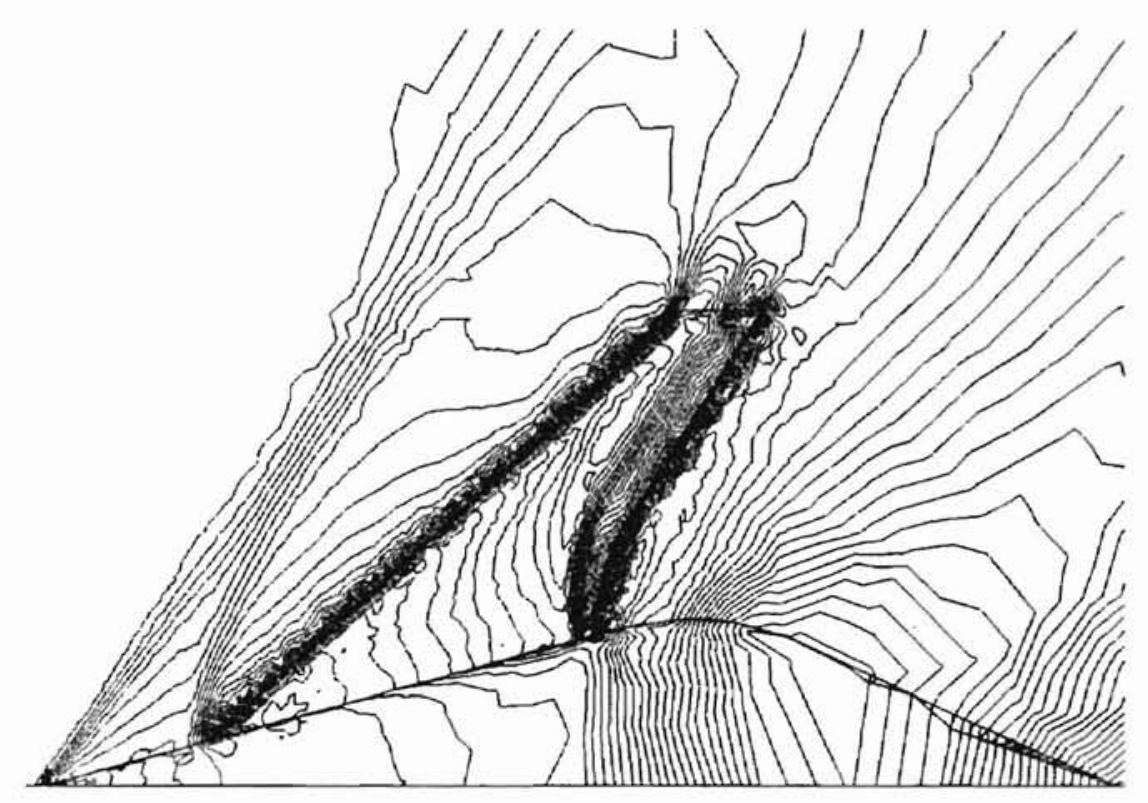

Figure 4.18. Pressure Contours Generated Using Steady Finite Element Euler Analysis at Mach 1.3

The perturbation method and the Piston method are used to determine the flutter boundary at Mach 1.3. These boundaries are determined by analyzing the transients of the different modes. Usually, the most obvious mode to determine the stability of the system is mode 1. For the perturbation and Piston methods, a half interval search is conducted to determine the stability of the system. In other words, an unsteady analysis using piston or P.S. is run at a given density. Say one transient is convergent at a given density and another is divergent at a different density. The value half way between the 
two densities is the new value used in another analysis. This analysis is repeated until a neutral transient in mode 1 is determined. Since a full transient takes only minutes to run, several transients may be run to pinpoint the flutter point for both methods. Next, these boundaries are compared with the flutter boundary determined by the unsteady Euler analysis. Since this analysis requires significantly more computation time, on the order of days, a logarithmic decrement of mode 1 is used to determine the modal damping ratio for each transient. Once enough transients are generated (usually 2-4), the damping ratio vs. Mach number points are fit with a cubic spline curve fit to determine where the curve crosses the neutral damping axis. This intersection is assumed to be the flutter point predicted by unsteady Euler analysis and is ideally the most accurate. Comparisons of analysis at Mach 1.3 are shown in Figure 4.19 below. The mode 1 transients for each density point are illustrated in Appendix C. on page 94.

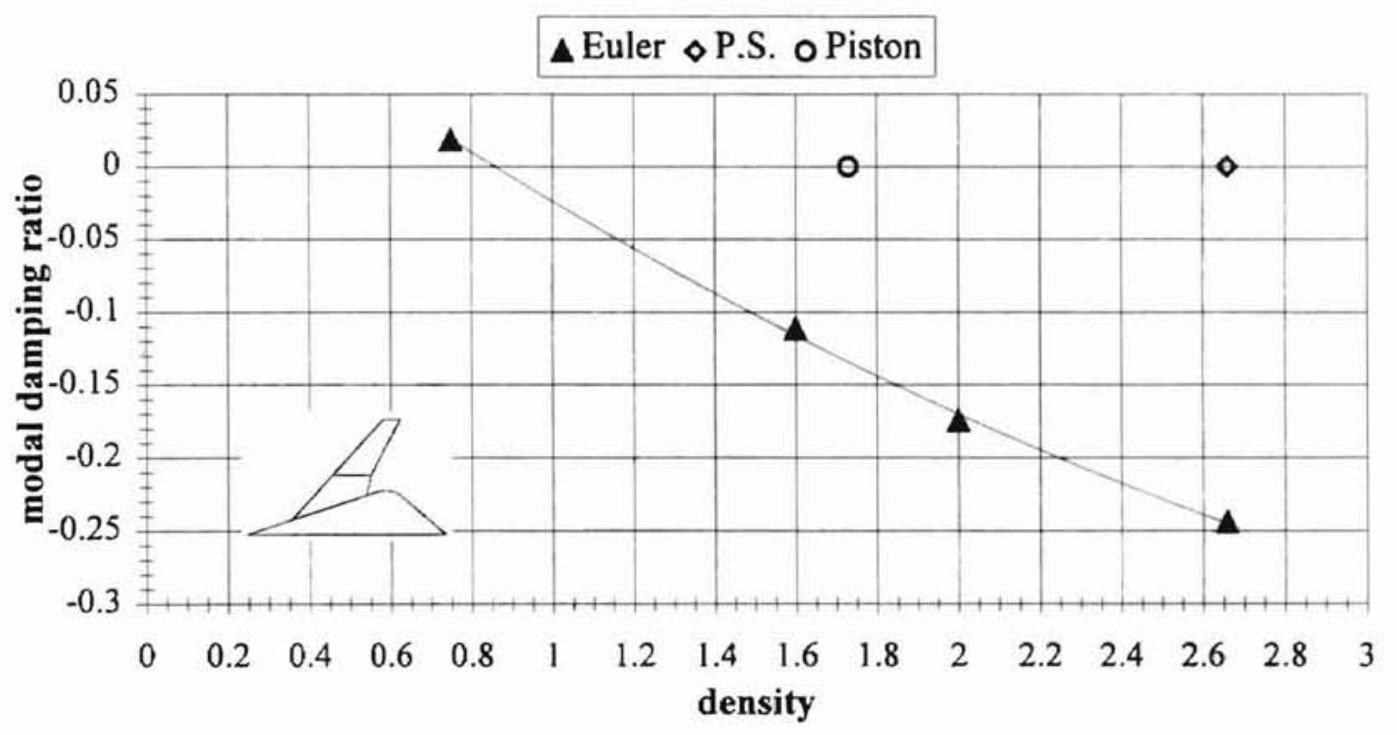

Figure 4.19. CSWC Flutter Boundary Analysis For Mach 1.3 
In Figure 4.19 above, the Euler analysis predicts a flutter point at about $\rho$ equal to 0.85 kilograms per meter cubed which is not at all close to the estimate given by the P.S. The piston method however, is considerably closer to the flutter point. This, however, is by chance since both methods give a very poor estimate at very low Mach. Thus far, this proves the assumptions previously discussed by Lighthill and Ashley that Mach number must be much greater than 1 for accurate results. A trend which gives a better understanding of this phenomenon is developed, graphed, and discussed later.

Next, the same analysis is performed at Mach 1.6. Note how the shock produced by the cone is swept back more at Mach 1.6 than Mach 1.3. This happens to be the design Mach number for this configuration. A plot of the steady pressure contours used as the mean flow conditions for the P.S. is shown in Figure 4.20.

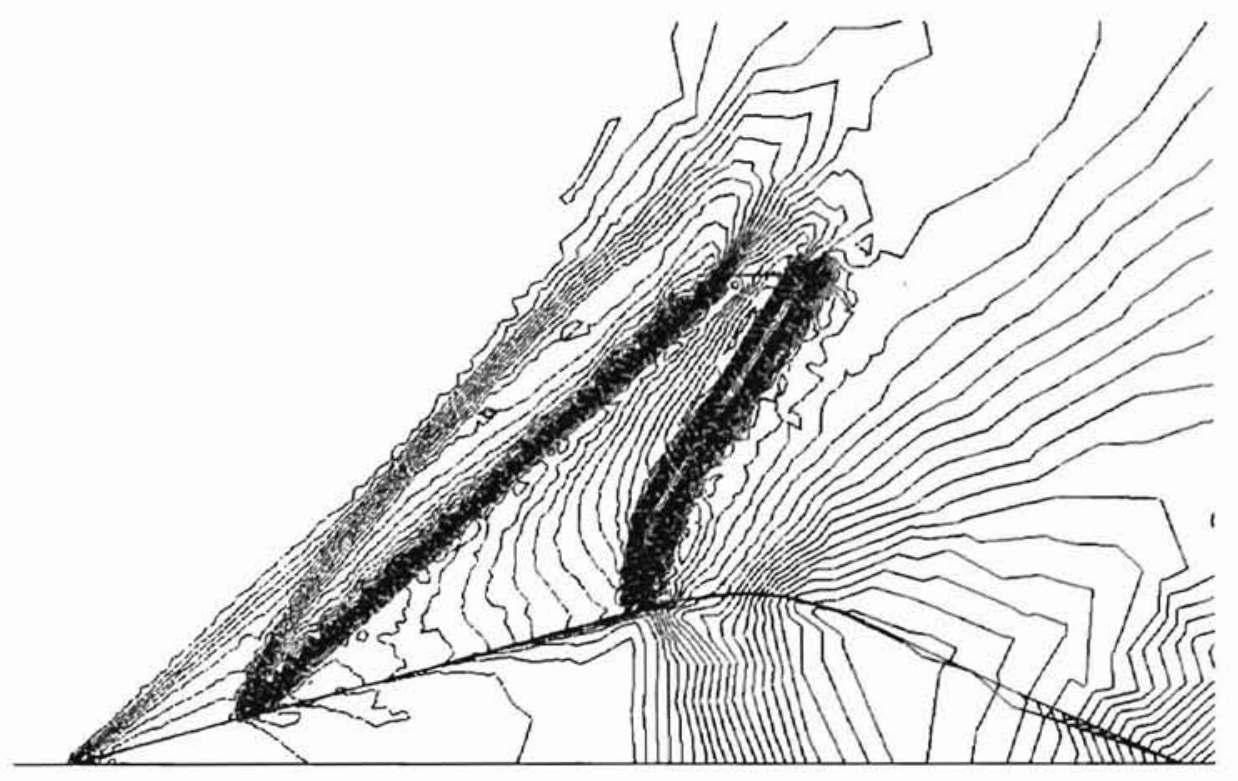

Figure 4.20. Pressure Contours Generated Using Steady Finite Element Euler Analysis at Mach 1.6 
Comparisons of analysis are done in the same manner as with Mach 1.3 above and is shown here in Figure 4.21.

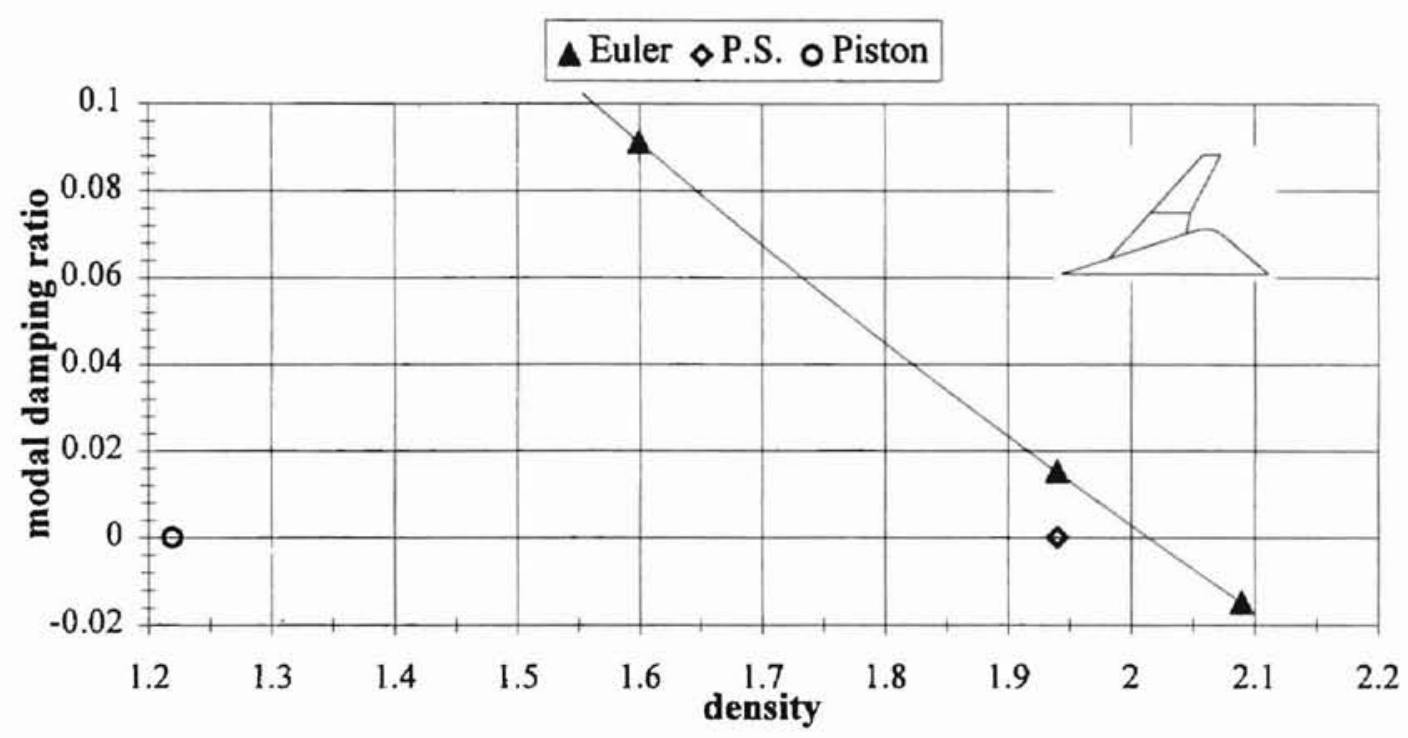

Figure 4.21. CSWC Flutter Boundary Analysis For Mach 1.6

As shown in Figure 4.21, the P.S. very closely predicts the flutter point determined by the unsteady Euler analysis. This again supports the assumptions made earlier that for this method to be accurate, the Mach number must be significantly greater than 1 .

The same analysis is repeated for Mach 2.0, Mach 2.4, and Mach 2.8 and shown in Figure 4.22. Notice the shock interactions in the mean flow for these Mach numbers. This is another example where the Perturbation method is able to capture the non-linear three-dimensional flow characteristics by accounting for these anomalies in the mean flow. 

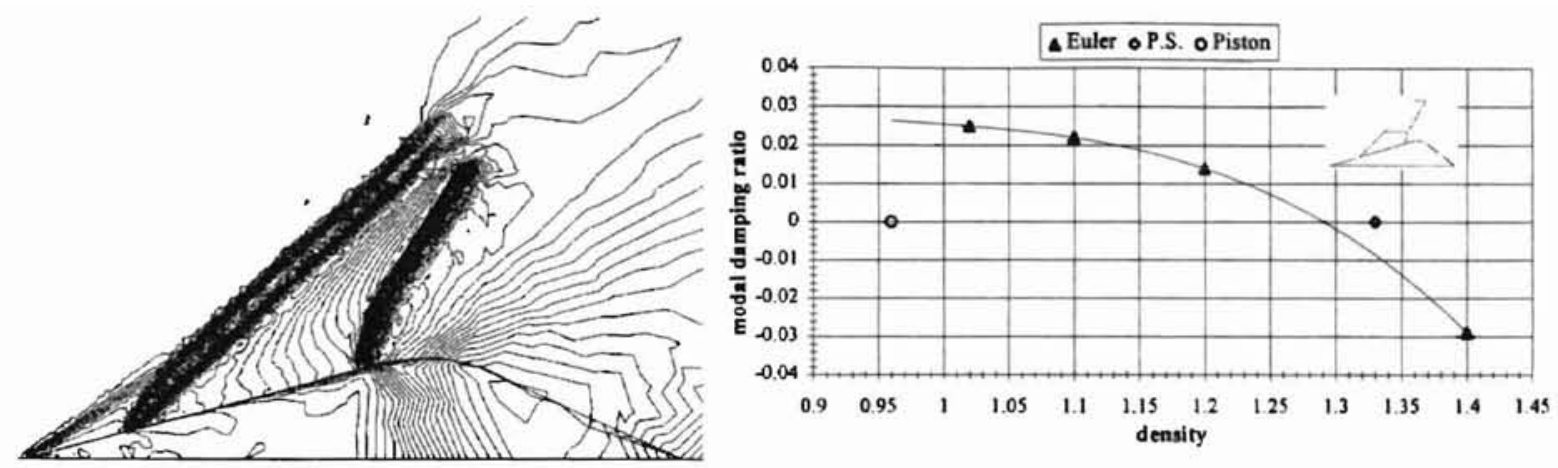

(a) Mach 2.0
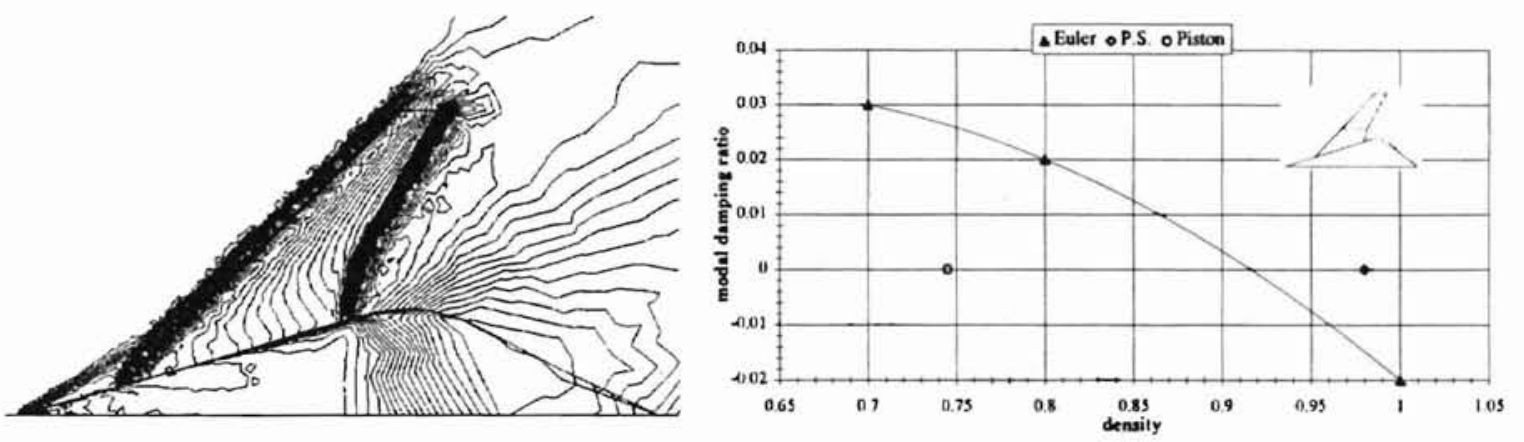

(b) Mach 2.4
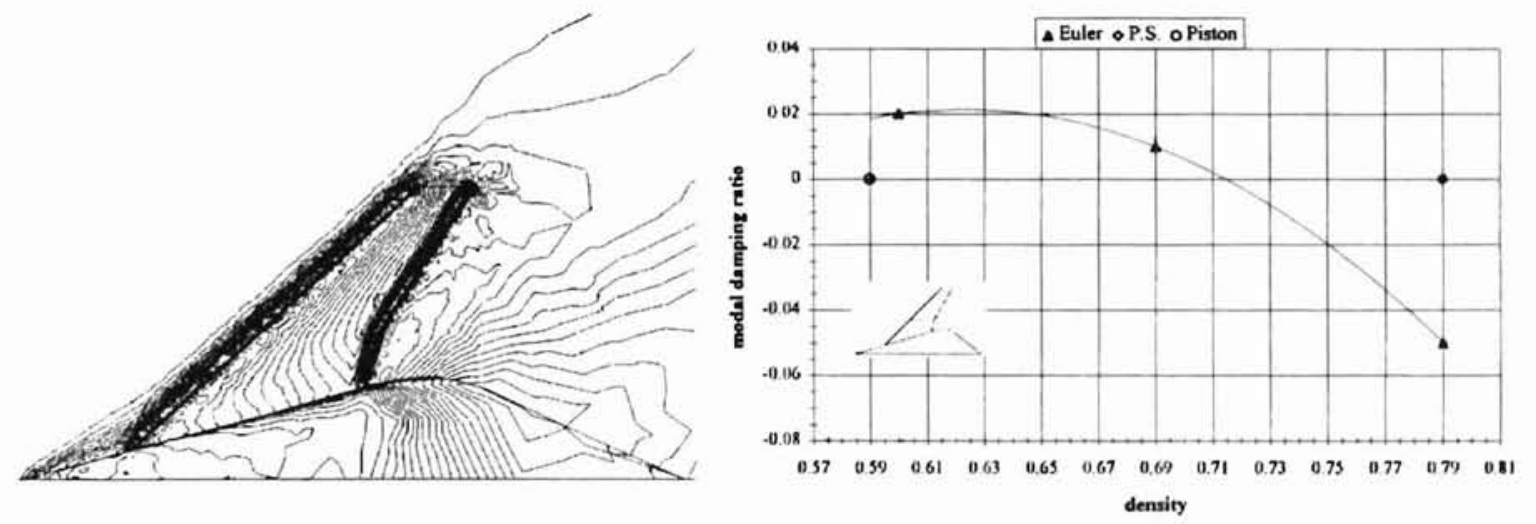

(c) Mach 2.8

Figure 4.22. CSWC Pressure Contours Generated Using Steady Finite Element Euler Analysis (left column) and Flutter Boundary Analysis (right column)

The flutter boundary over the whole Mach range is shown below in tabular form (Table 4.4) and illustrated in Figure 4.23 and Figure 4.24. : 
Table 4.4. CSWC Flutter Boundary Data

\begin{tabular}{|c|c|c|c|c|c|}
\hline & Euler & \multicolumn{2}{|c|}{ Perturbation Solution } & \multicolumn{2}{|c|}{ Piston Theory } \\
\hline \hline $\mathrm{M}$ & $\rho\left(\mathbf{k g} / \mathbf{m}^{\wedge} \mathbf{3}\right)$ & $\rho\left(\mathbf{k g} / \mathbf{m}^{\wedge} \mathbf{3}\right)$ & \% Error & $\rho\left(\mathbf{k g} / \mathbf{m}^{\wedge} \mathbf{3}\right)$ & $\%$ Error \\
\hline 1.3 & 0.85 & 2.66 & 213 & 1.73 & 104 \\
1.6 & 2.015 & 1.94 & 4 & 1.22 & 39 \\
2.0 & 1.29 & 1.33 & 3 & 0.96 & 26 \\
2.4 & 0.92 & 0.98 & 7 & 0.745 & 19 \\
2.8 & 0.72 & 0.79 & 10 & 0.59 & 18 \\
\hline
\end{tabular}

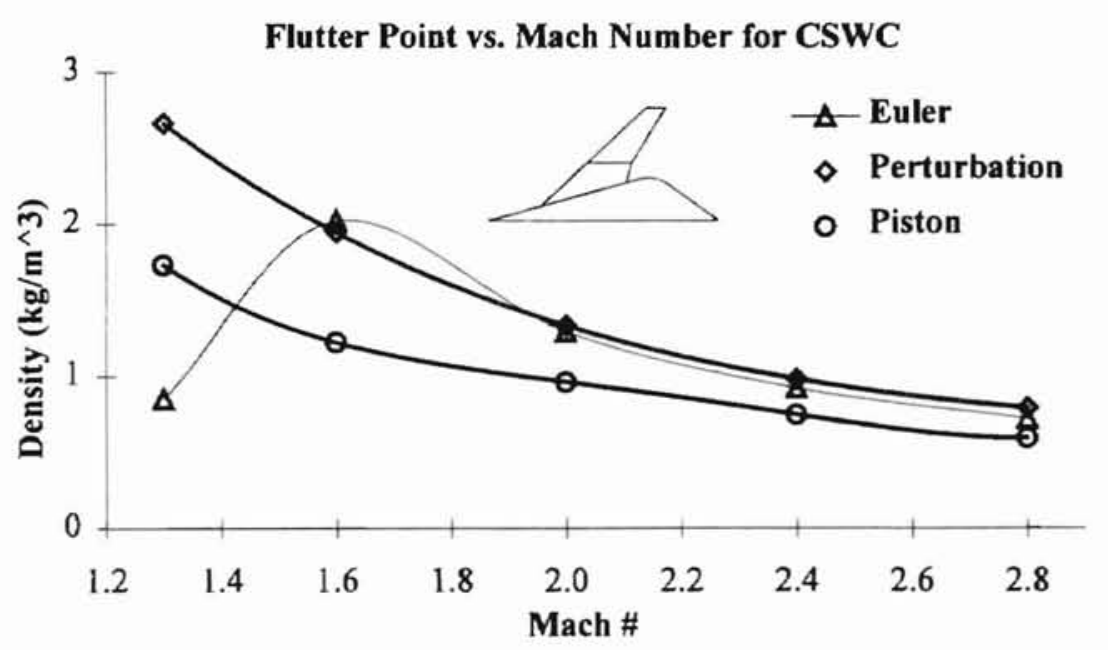

Figure 4.23. CSWC Flutter Boundary

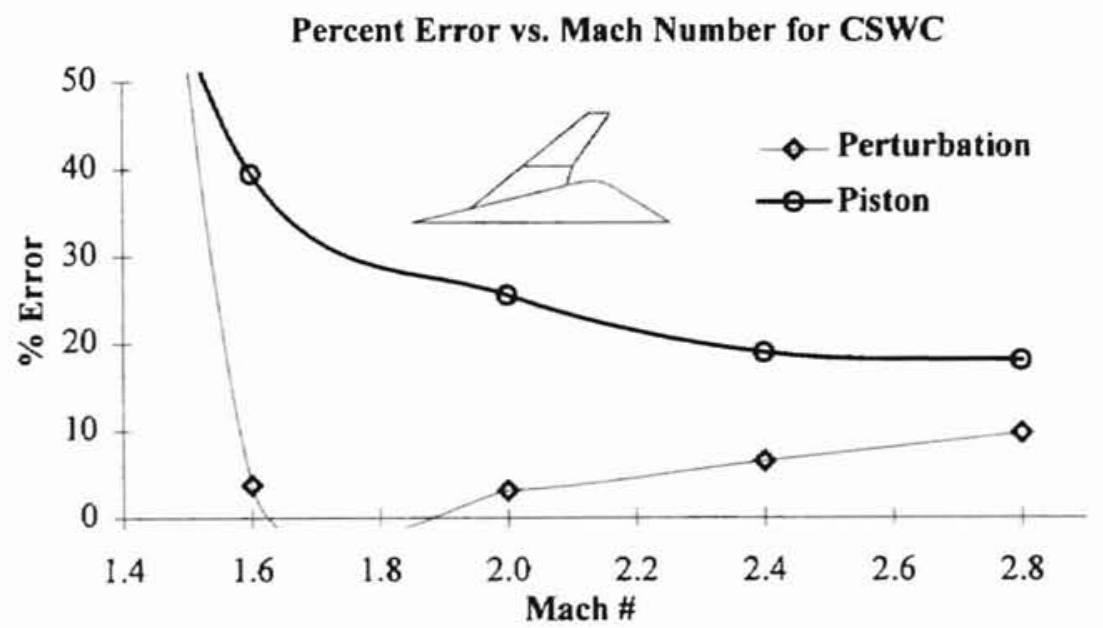

Figure 4.24. CSWC Flutter Boundary \% Error Analysis 
The graphical representations in Appendix A on page 60 show very similar trends to the data just illustrated. Based on the data in Appendix A, it stands to reason that as the Mach number increases, the error via the P.S. remains low whereas the error via piston theory significantly increases. 


\section{CHAPTER 5}

\section{CONCLUSIONS AND RECOMMENDATIONS}

\section{Conclusions}

Obtained results reveal that the goal to enhance the practicality of time-marching supersonic flutter analysis has been achieved. This was accomplished by taking advantage of the efficient aspects of the supersonic modeling technique known as the piston theory and applying it as a small perturbation to a given mean flow obtained by the steady finite element Euler analysis in STARS. By replacing the unsteady Euler equations with this modeling technique, the results of the unsteady aerodynamic analysis are produced relatively instantaneously with very little error. This was previously shown in the aeroelastic analysis of the GHV.

Several supersonic modeling techniques were considered to be implemented into STARS but the piston theory applied as a perturbation to the mean flow proves to be the most feasible. The feasibility of this method is a result of the simplicity and easy implementation into an existing aeroelastic analysis computer program. Since all the unsteady wave equation needs to be applied as a perturbation to an existing steady mean 
flow is the local conditions and the perturbed outward normal unit vector, easy and accurate implementation into an existing computer code with these conditions may be achieved. Furthermore, by complying with the assumptions and limitations of this method, and taking advantage of the small perturbations associated with aeroelastic analysis, the P.S. has proven extremely accurate over a considerably large Mach range.

In addition to the Perturbation Solution's ability to handle a number of geometrical configurations over a wide Mach range, it is also very accurate in areas where non-linearities such as shock interactions occur. In fact, since these areas of shock interaction are associated with strong mean flow characteristics, this method really "shines" since the assumptions for validity are high Mach number and small perturbation from the mean flow. The rigid duct with an elastic plate previously discussed shows these results.

An added advantage of the presented approach is that the same grid may be used for the steady CFD solution, the perturbation model, and the time marching CFD solution. This is significant due to the fact that it takes on the order of hours and in many cases days to obtain a mean flow solution for a given geometry. 


\section{Recommendations}

Since the aeroelastic analysis performed on the CSWC seems to follow a very similar trend with that of the steady perturbation analysis shown in Appendix A on page 60 , it is highly recommend that validation at Mach numbers greater than Mach 2.8 be accomplished. Since the steady perturbation analysis shows very small error up to around Mach 8, it is assumed that the P.S. method with respect to the aeroelastic analysis will be very accurate as well.

A method initially presented as the "tag method" was briefly discussed but not looked into much further do to its probable inability to recognize areas of heavy shock interaction in its analysis. This method also takes advantage of the already existing mean flow generated by Euler analysis, not as a mean flow for a small perturbation, but as comparison at every nodal point in the computational domain with several supersonic and hypersonic modeling techniques. This is done by calculating the pressure for these modeling techniques and comparing with the pressure of the nodal location. The modeling technique which predicts the pressure the closest to that point "wins", is tagged to that point, and used for further analysis. Since many supersonic configurations are designed to avoid such anomalies as significant shock interactions, it is recommended that the "tag method" be given further consideration and compared with the P.S. method.

Also, an improvement in the method in which the damping ratio for the finite element unsteady Euler analysis performed is needed. At the moment, a logarithmic decrement is performed and on mode 1 which under certain circumstances is very difficult. 
Lastly, since the assumptions and limitations of the Piston Perturbation method are known, (i.e. $0.2<$ pressure ratio 3.5 ), an accuracy check based on these limitations is recommended. This analysis would need to determine the amount the pressure has been perturbed from the mean flow. Then, based on the previous assumptions, a rough error analysis may be performed on the perturbations which exceed these restrictions. This should not be too difficult a task and should aid in the efficient and accurate determination of a given flutter point. 


\section{BIBLIOGRAPHY}

Allen, David H. and Walter E Haisler, Introduction To Aerospace Structural Analysis, Wiley, New York, 1985.

Anderson, John D., Jr., Modern Compressible Flow With Historical Perspective, McGraw-Hill, New York, 1982.

Ashley, Holt, and Garabed Zartarian, "Piston Theory-A New Aerodynamic Tool for the Aeroelastician," Presented at the Twenty-Forth Annual Aeroelasticity Meeting, New York, January, 1956.

Bertin, John J. and Michael L. Smith, Aerodynamics For Engineers, $2^{\text {nd }}$ Edition, PrenticeHall, New Jersey, 1989.

Bonner, E.; Cleaver, W.; and Dunn, K., "Aerodynamic Preliminary Analysis System II. Part I Theory", NASA CR-165627, 1981.

Cruz, Christopher I. and Gregory J. Sova, "Improved Tangent-Cone Method for the Aerodynamic Preliminary Analysis System (APAS) Version of the Hypersonic Arbitrary-Body Program", NASA TM 4165, February, 1990.

Dowel, E.H., et al, A Modern Course in Aeroelasticity, $3^{\text {rd }}$ Edition, Klewer Academic Publishers, 1995.

Fung, Y. C., An Introduction to the Theory of Aeroelasticity, $1^{\text {st }}$ Edition, Dover, New York, 1969.

Gupta, K.K., "STARS - An Integrated General-Purpose Finite Element Structural, Aeroelastic, and Aeroservoelastic Analysis Computer Program," NASA TM101709, June, 1990.

Gupta, K.K., Peterson, K., and Lawson, C., "Multidisciplinary Modeling and Simulation of a Generic Hypersonic Vehicle," AIAA-91-5015, AIAA $3^{\text {rd }}$ International Aerospace Planes Conference, Orlando, FL, December, 1991. 
Gupta, K.K., Peterson, K., and Lawson, C., "On Some Recent Advances in Multidisciplinary Analysis of Hypersonic Vehicles," ALAA-92-5026, ALAA Fourth International Aerospace Planes Conference, Orlando, FL, December, 1991.

Inman, Daniel J., Engineering Vibration, Prentice-Hall, New Jersey, 1994.

John, James E. A., Gas Dynamics, $2^{\text {nd }}$ Edition, Allyn and Bacon, Boston, 1984.

Lighthill, M. J., "Oscillating Airfoils at High Mach Number", Journal of the Aeronautical Sciences, vol. 20, No. 6, pp. 402-406, June, 1953.

Morgan, Homer G., Harry L. Runyan, and Vera Huckel, "Theoretical Considerations of Flutter at High Mach Numbers," Presented at the Aeroelasticity-II Session, Twenty-Sixth Annual Meeting, New York, January, 1958.

Marsden, Jerrold E. and Anthony J. Tromba, Vector Calculus, $4^{\text {th }}$ Edition, Freeman, New York, 1996.

Moran, Michael J. and Howard N. Shapiro, Fundamentals Of Engineering Thermodynamics, $2^{\text {nd }}$ Edition, Wiley, New York, 1992.

Neice, Mary M., "Tables and Charts of Flow Parameters Across Oblique Shocks", NACA TN 1673, August, 1948.

Pittman, Jimmy L. (appendix by C. L. W. Edwards): "Application of Supersonic Linear Theory and Hypersonic Impact Methods to Three Nonslender Hypersonic airplane Concepts at Mach Numbers From 1.10 to 2.86", NASA TP-1539, 1979.

Sims, Joseph L., "Tables for Supersonic Flow Around Right Circular Cones at Zero Angle of Attack", NASA SP-3004, 1964.

Truitt, Robert W., Hypersonic Aerodynamics, Ronald Press Co., 1959. 
APPENDICES 


\section{APPENDIX A: COMPARISON DATA}

Note, in the following charts, "Newt" is Newtonian, "Pist" is the P.S. method, "Piston" is straight piston, and "cone" is tangent cone. Also, "Cpo" is the mean flow pressure coefficient and " $\mathrm{Cp}_{\mathrm{i}}$ " is the final exact pressure coefficient. Finally, a straight line represents the nearest value to the exact value or lowest percent error.

\section{Wedge in Compression}
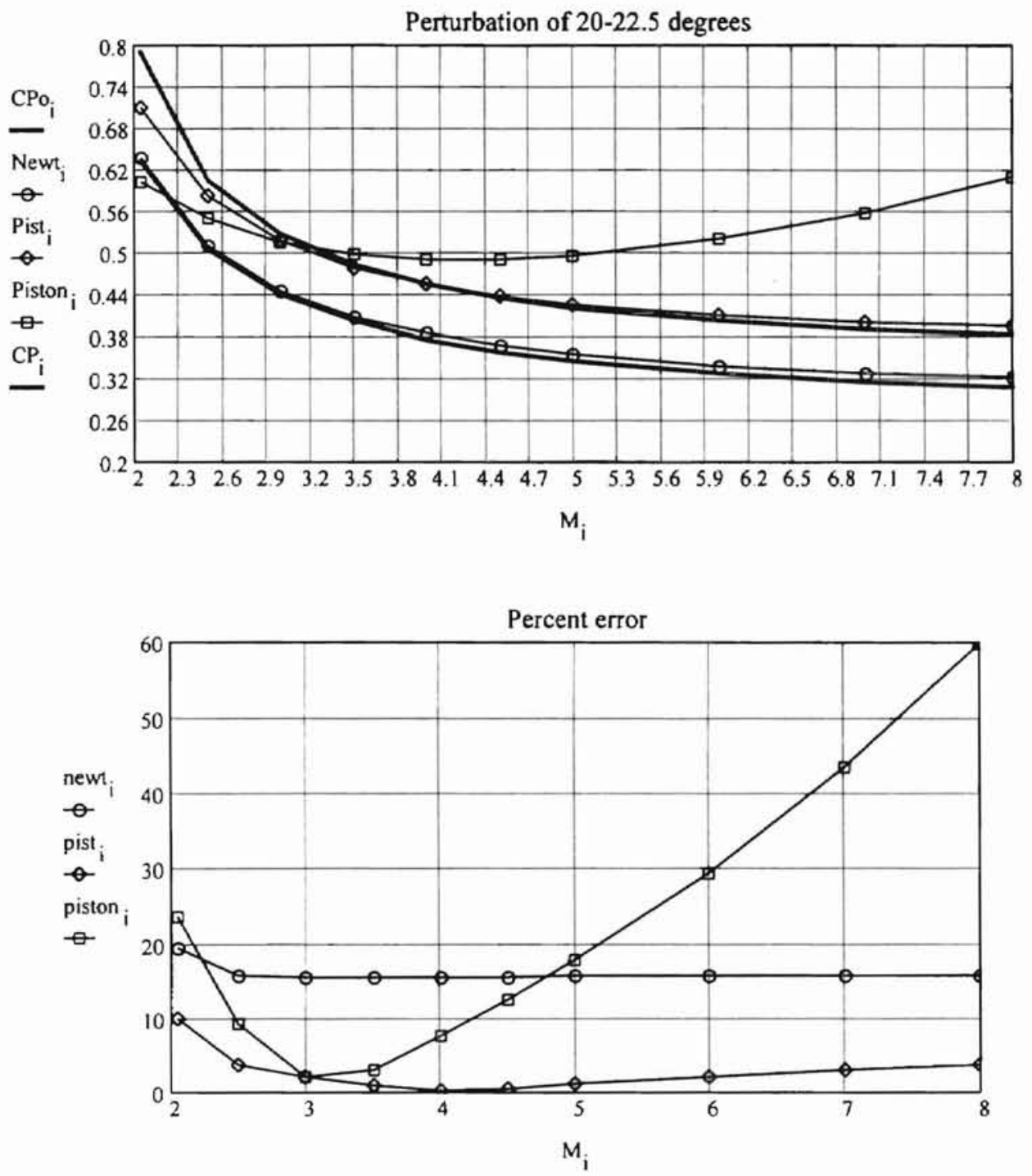

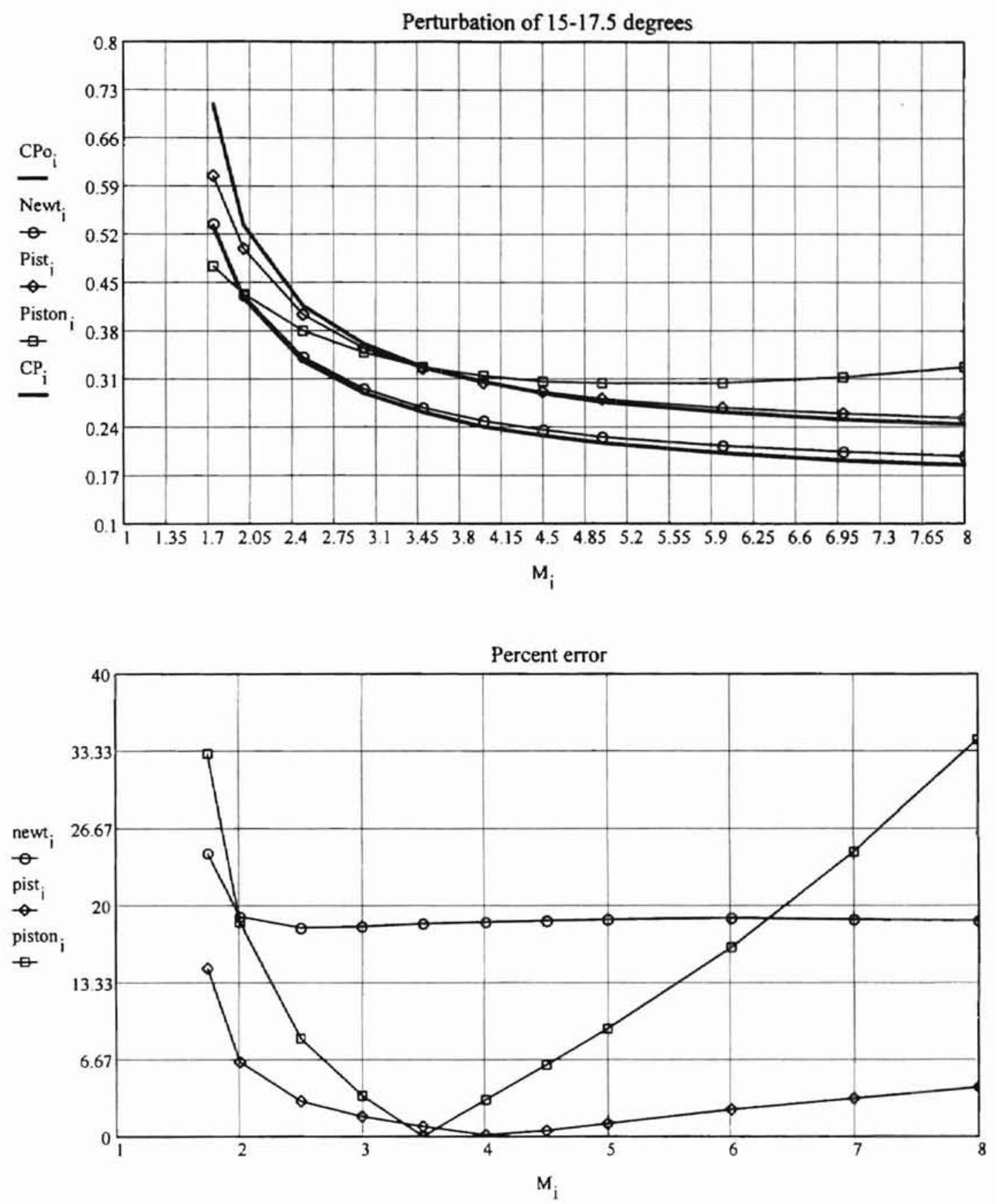

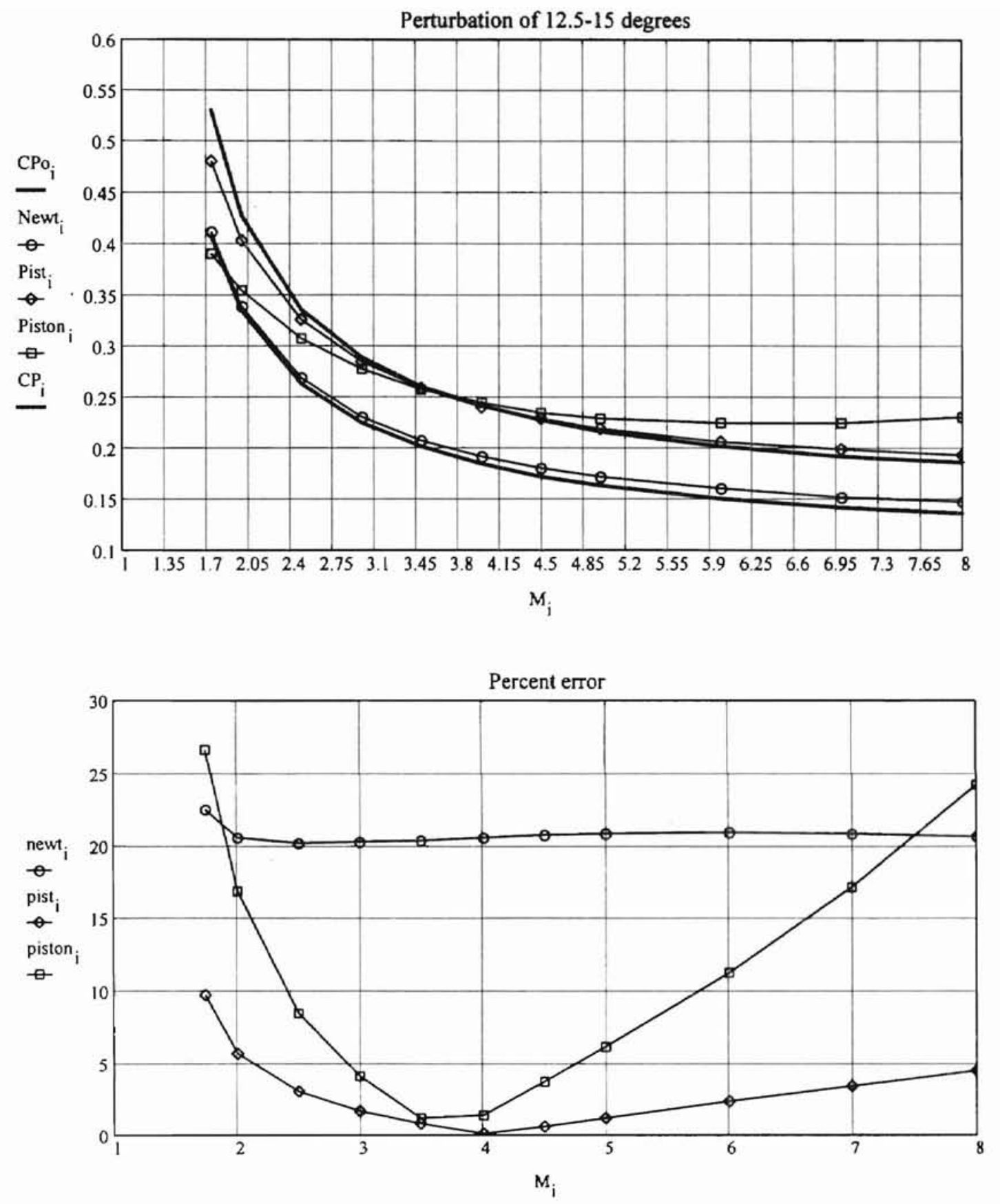

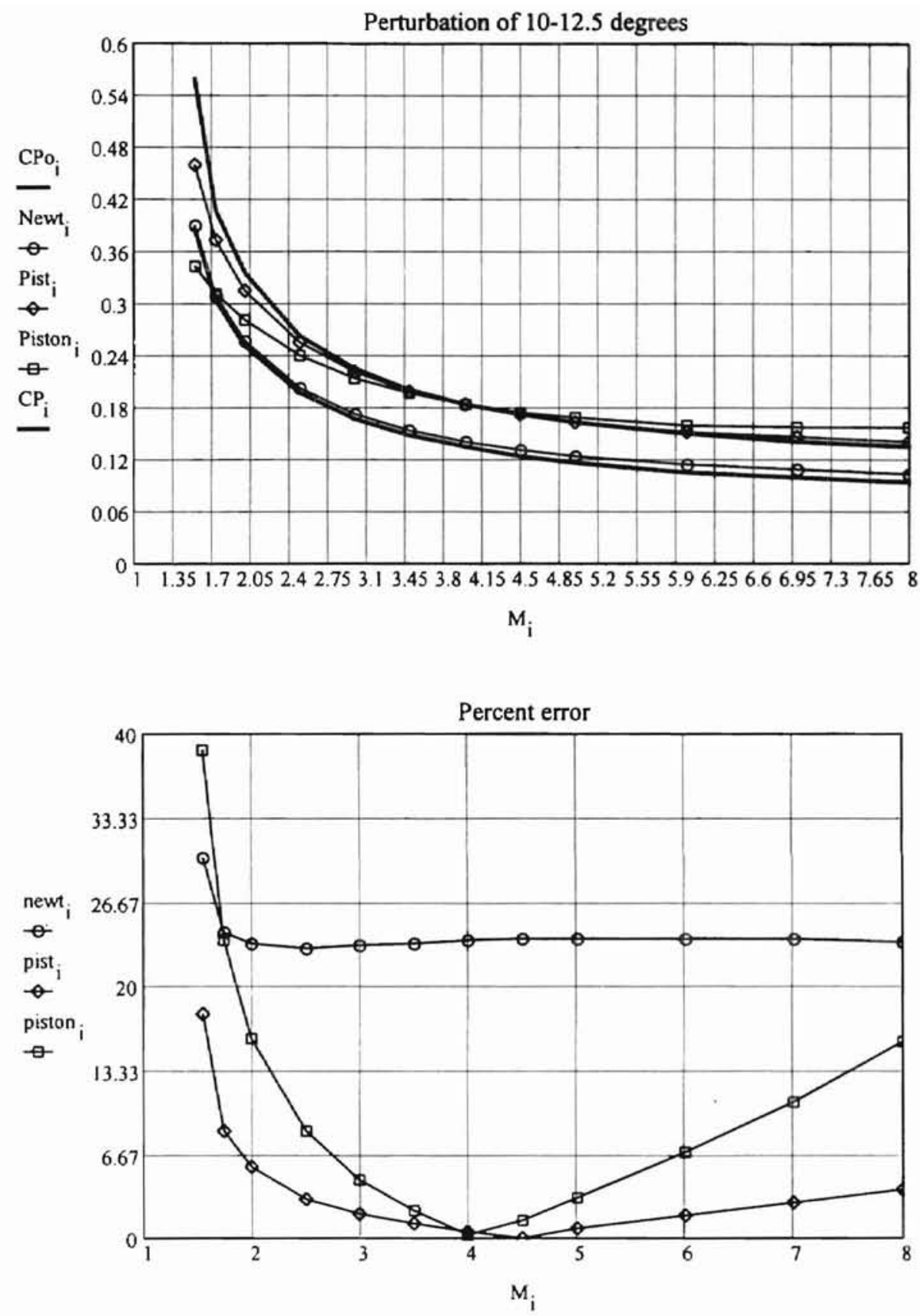

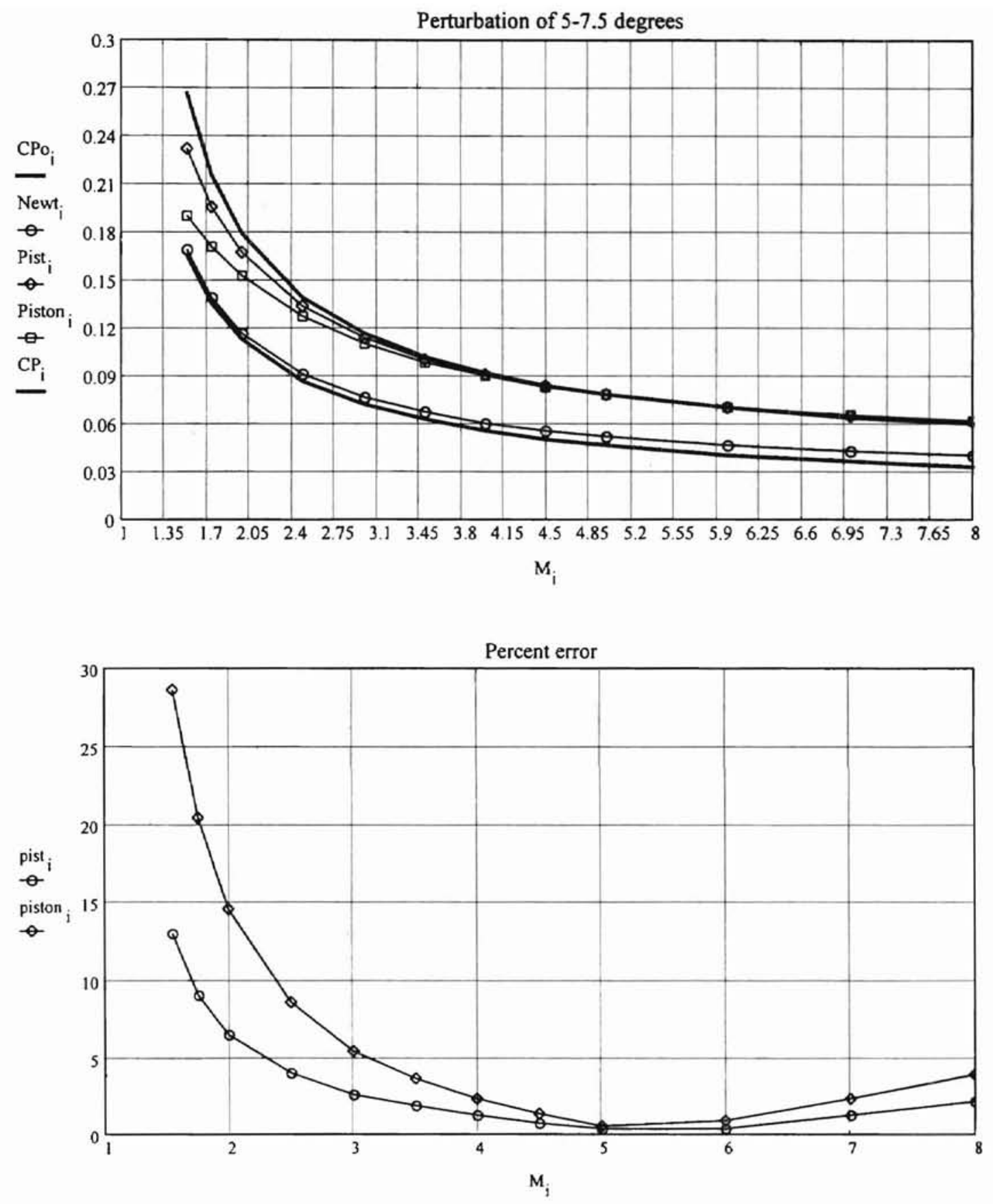


\section{Wedge in Expansion}

Note, in the following charts, "Newt" is Newtonian, "Pist" is the P.S. method, "Piston" is straight piston, and "cone" is tangent cone. Also, "Cpo;" is the mean flow pressure coefficient and " $\mathrm{Cp}_{\mathrm{j}}$ " is the final exact pressure coefficient. Finally, a straight line represents the nearest value to the exact value or lowest percent error.
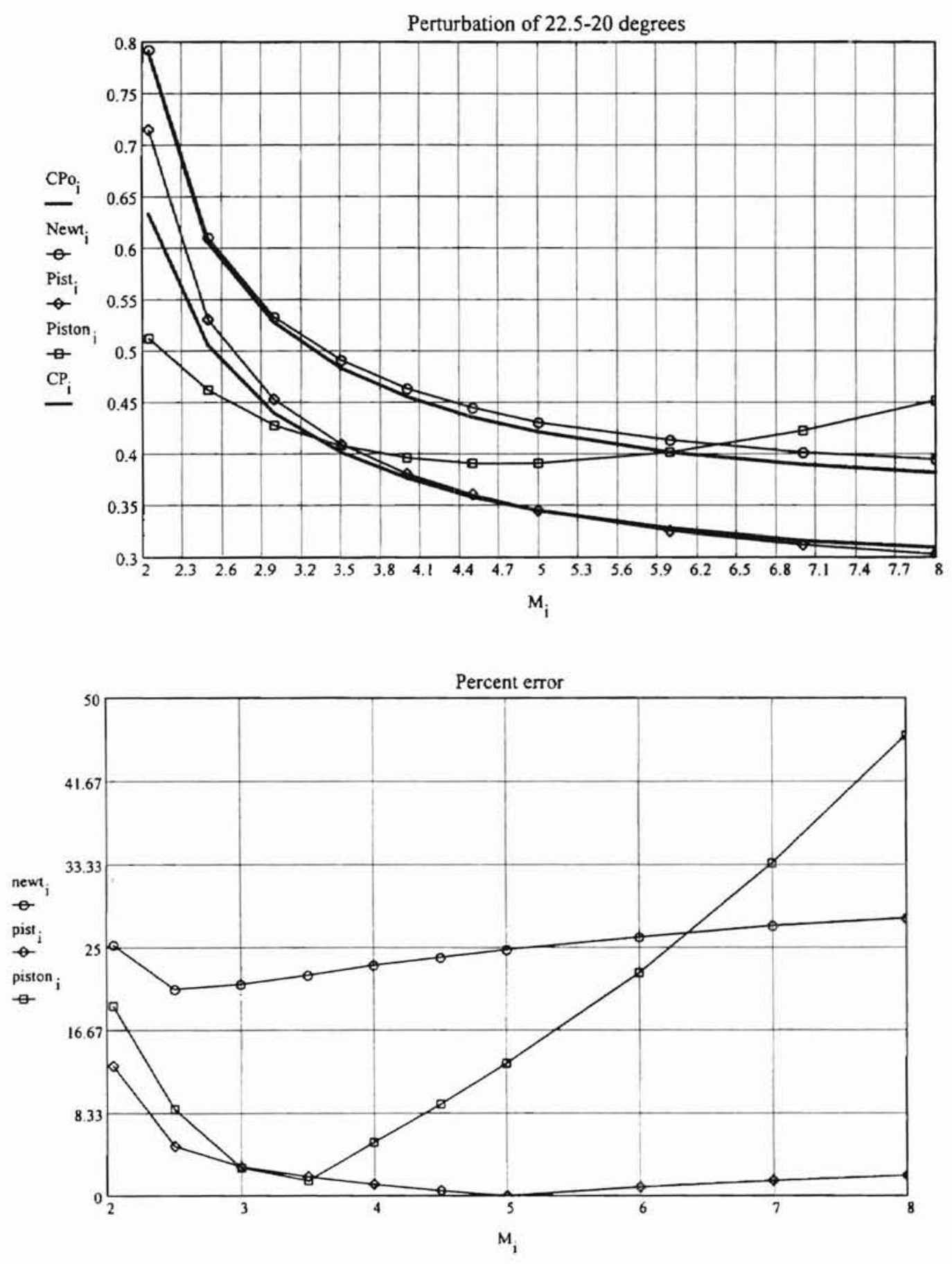

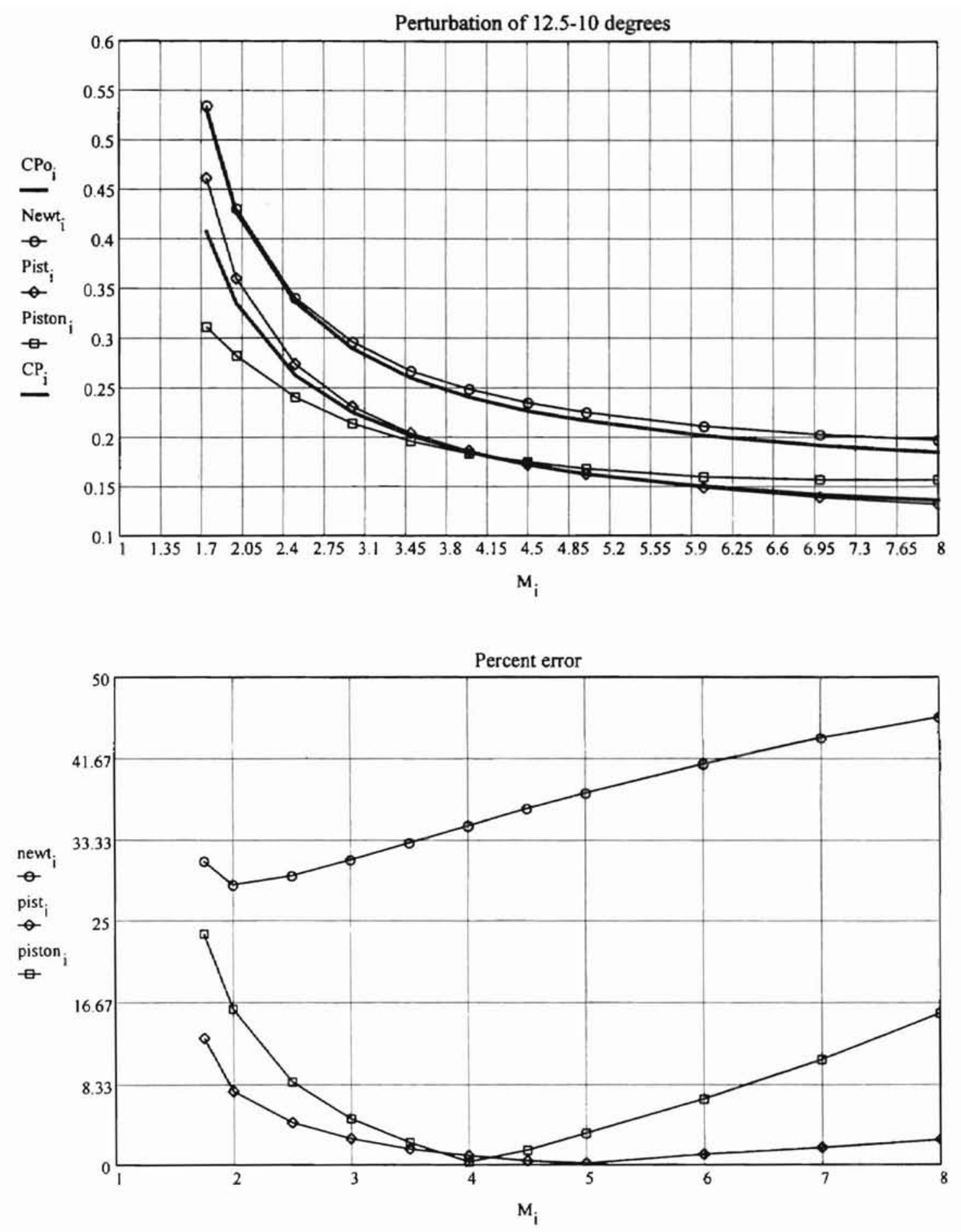

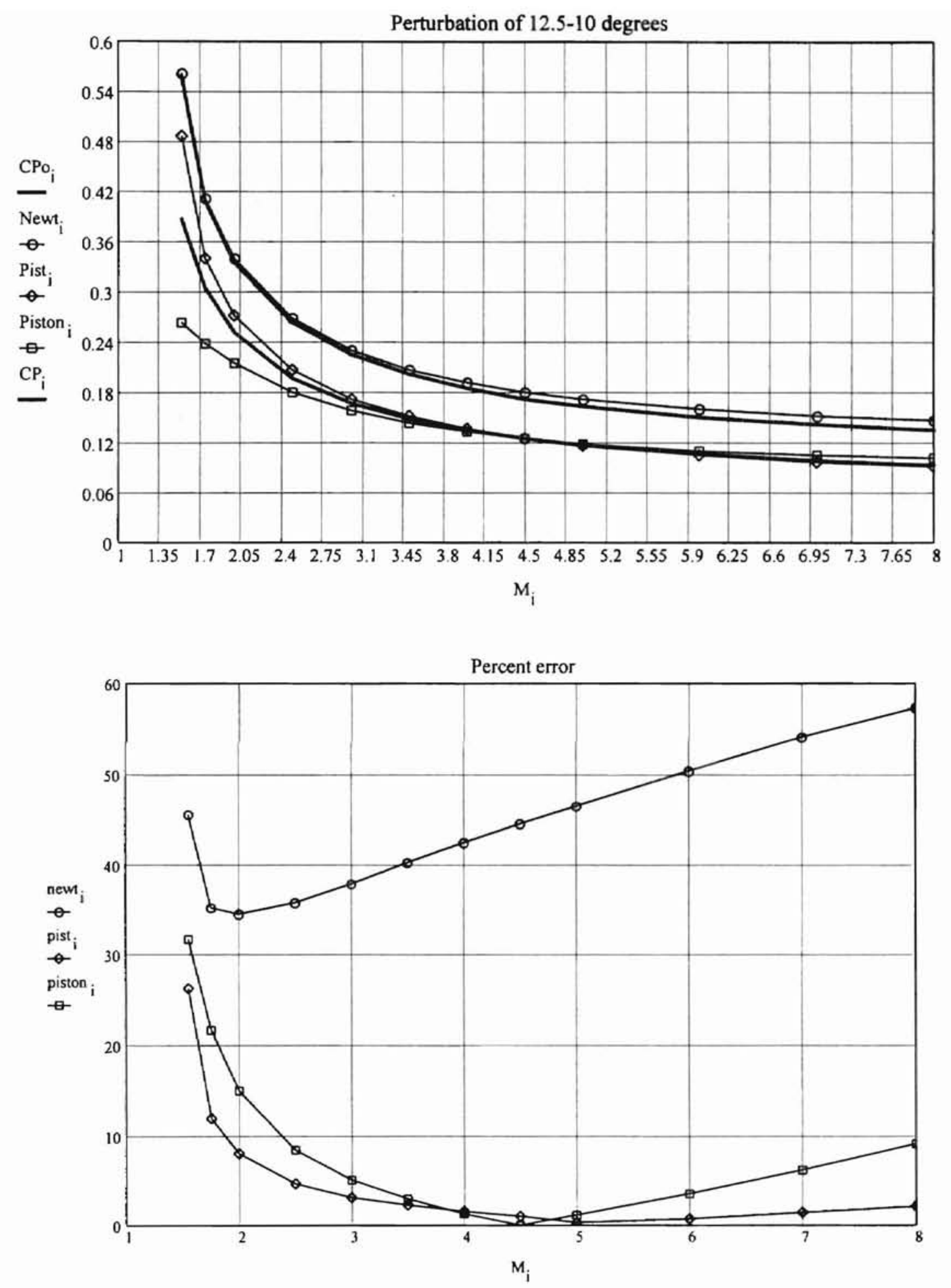

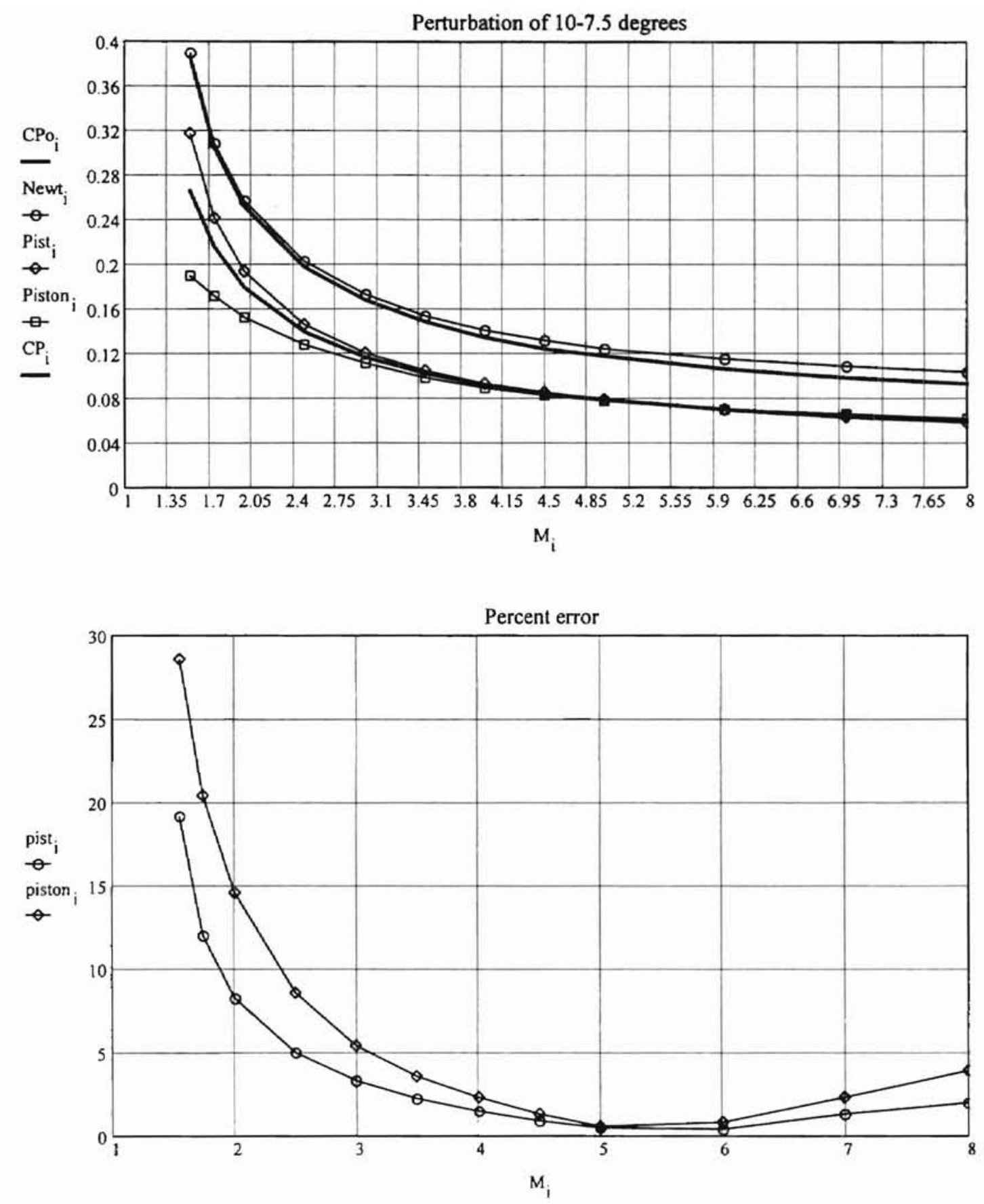

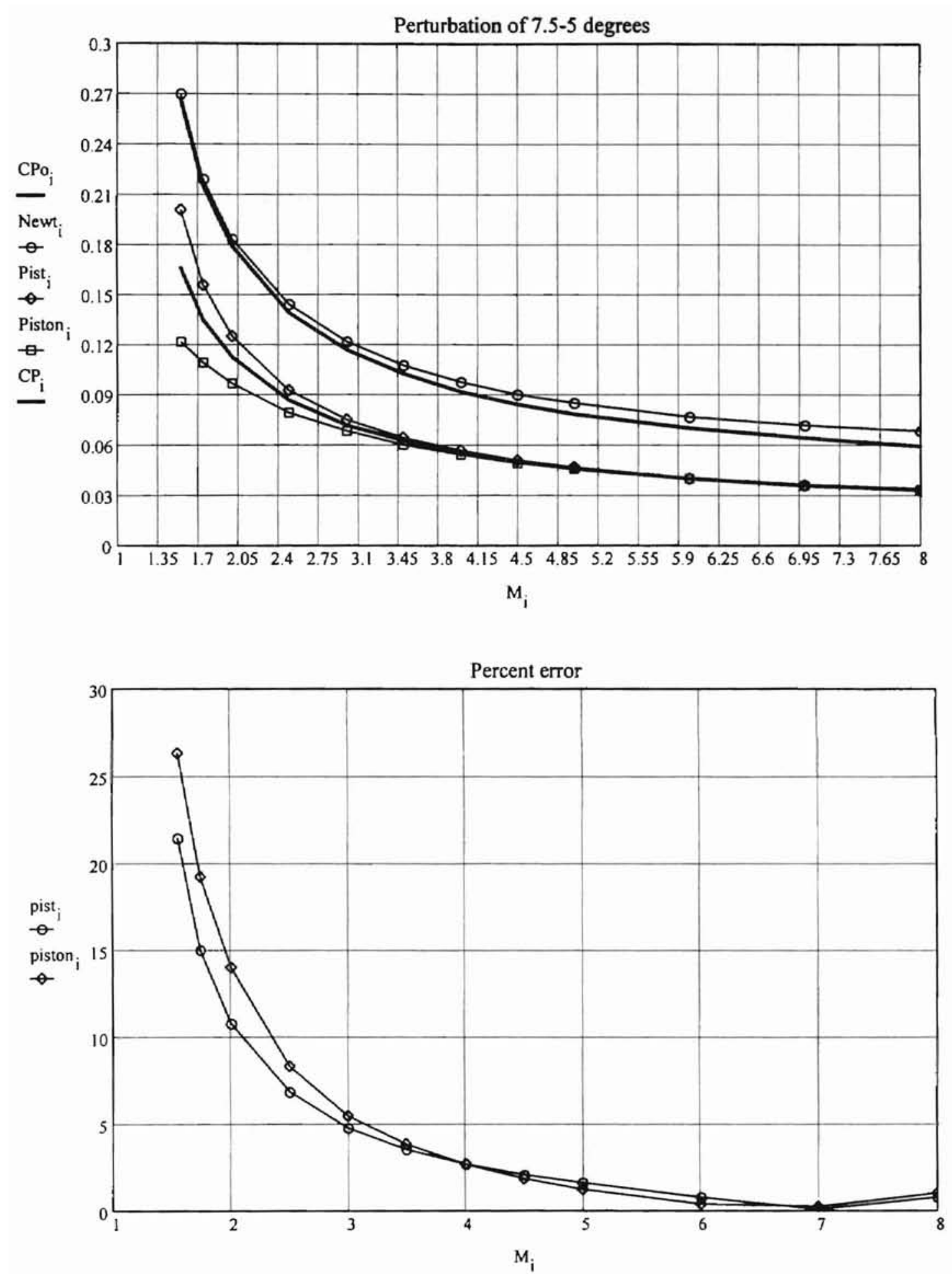


\section{Cone in Compression}

Note, in the following charts, "Newt" is Newtonian, "Pist" is the P.S. method, "Piston" is straight piston, and "cone" is tangent cone. Also, "Cpo" is the mean flow pressure coefficient and " $\mathrm{Cp}_{\mathrm{i}}$ " is the final exact pressure coefficient. Finally, a straight line represents the nearest value to the exact value or lowest percent error.
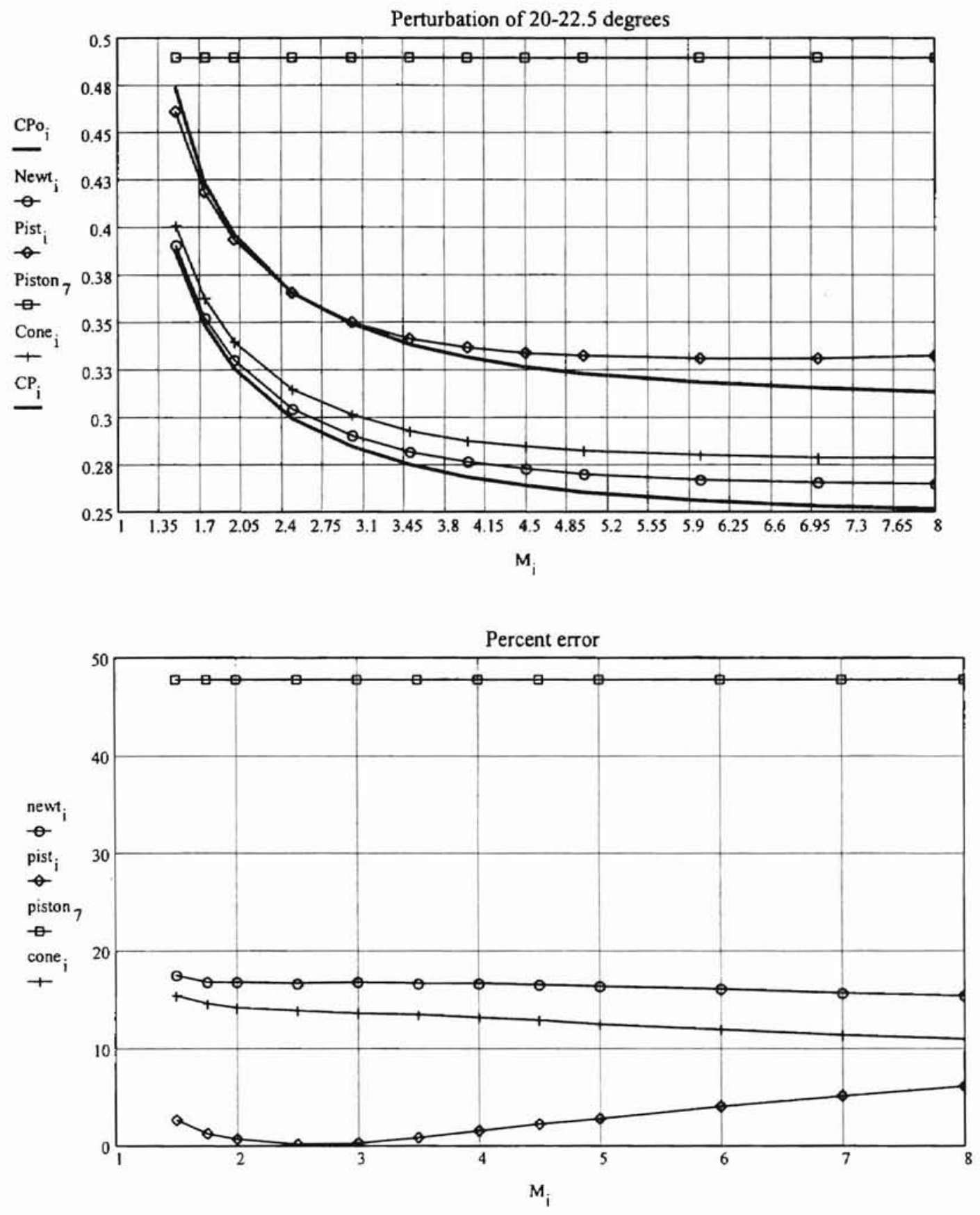
Perturbation of 15 to 17.5 degrees
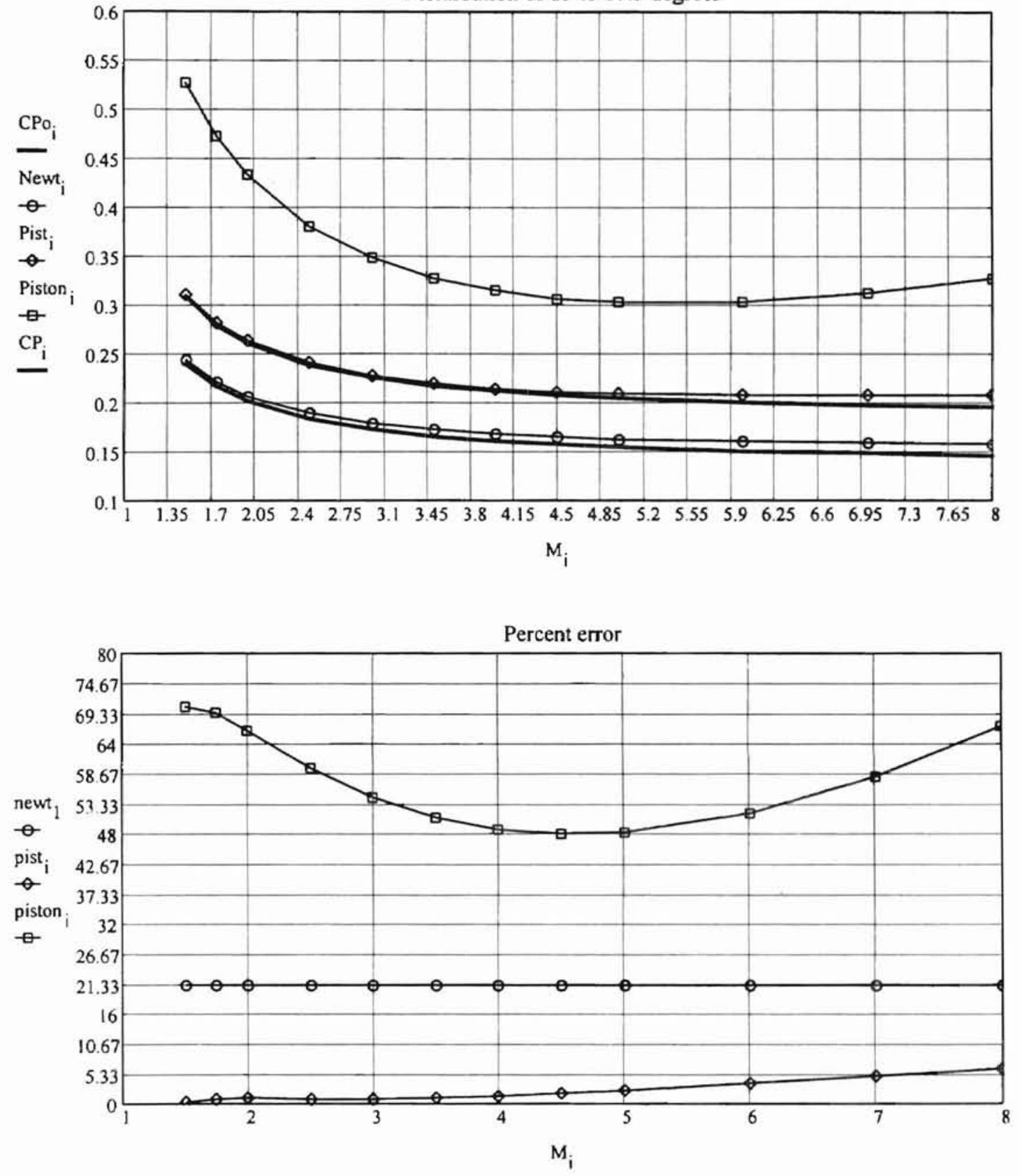
Perturbation of 12.5 to 15 degrees
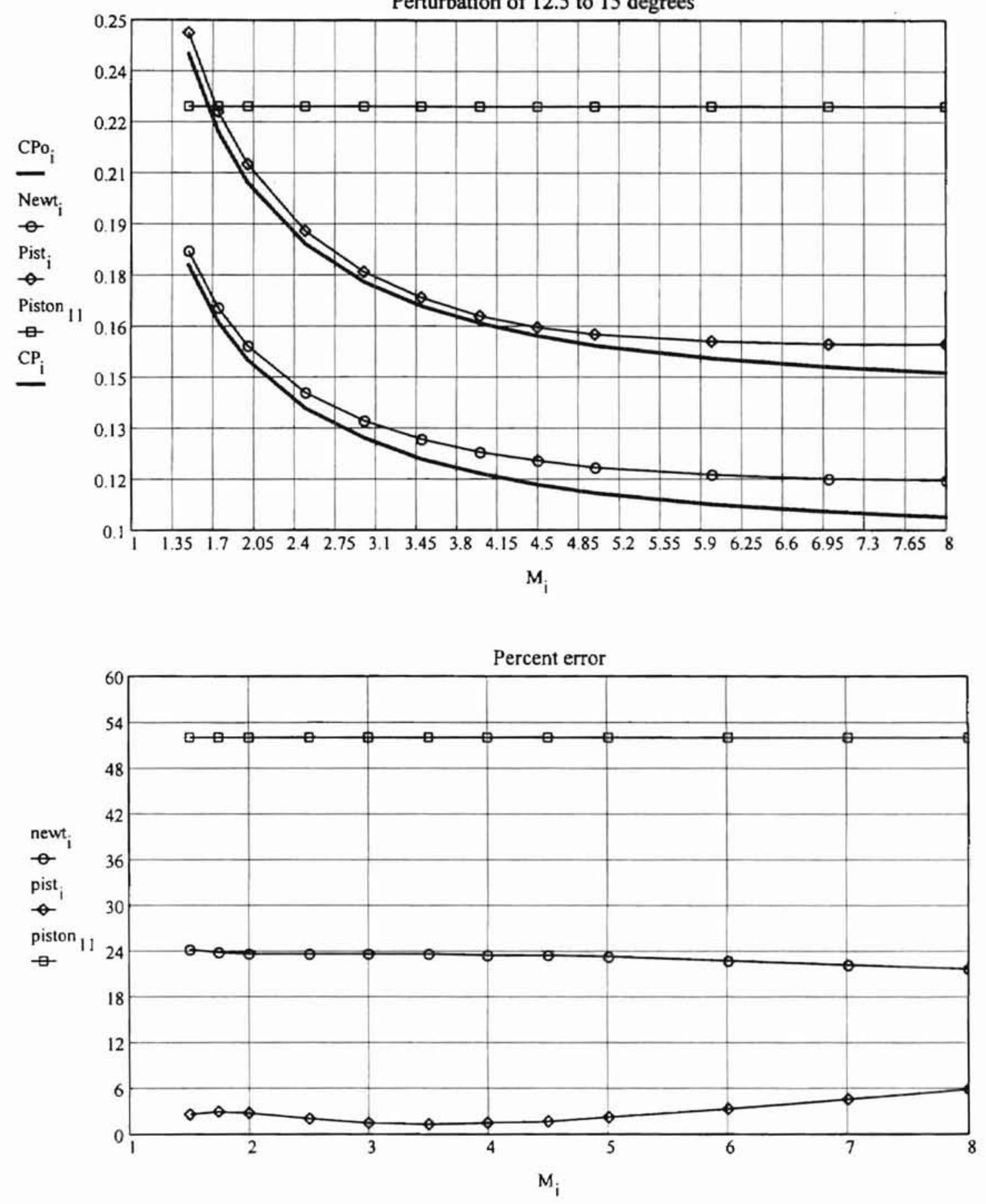

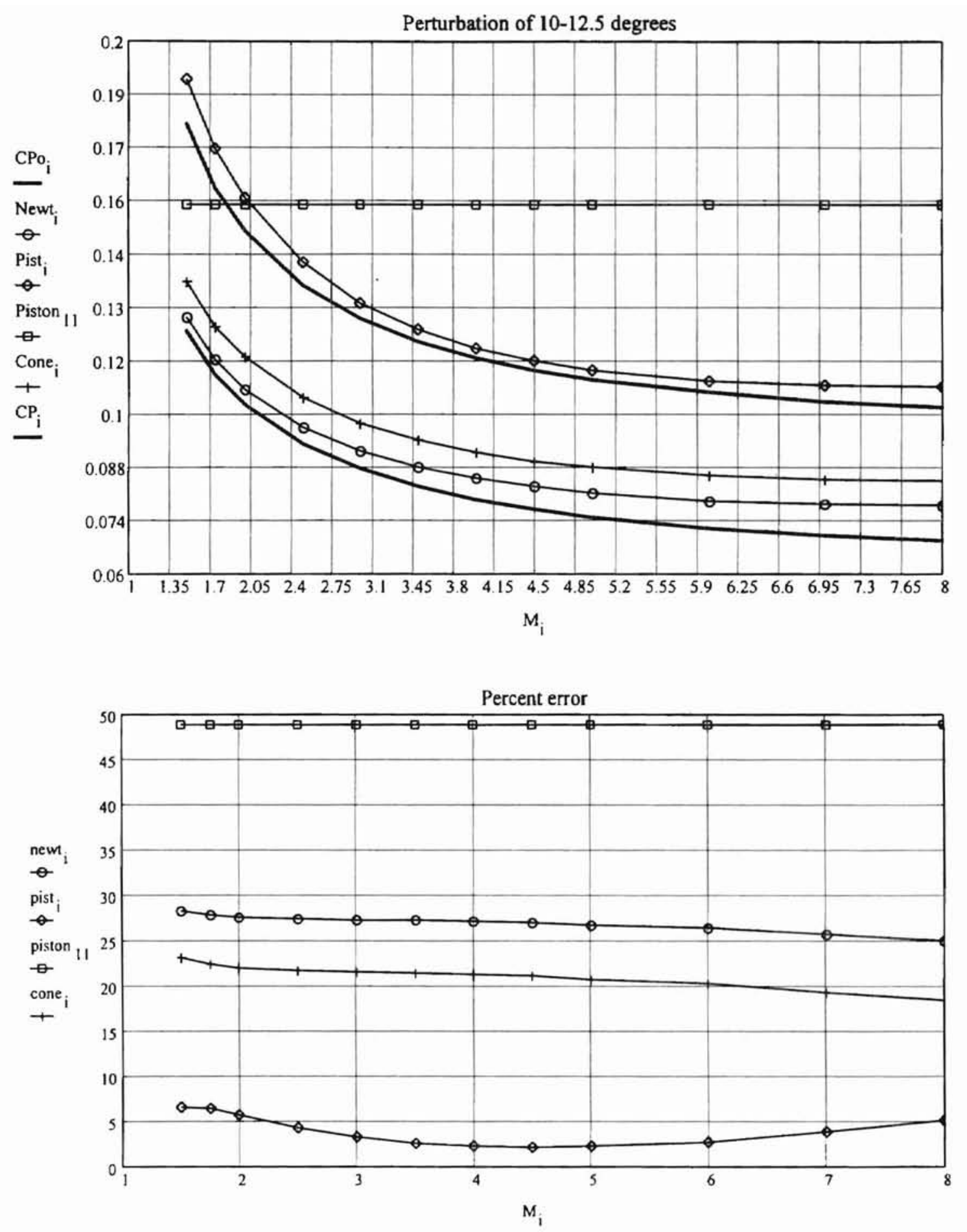
Perturbation of 5-7.5 degrees
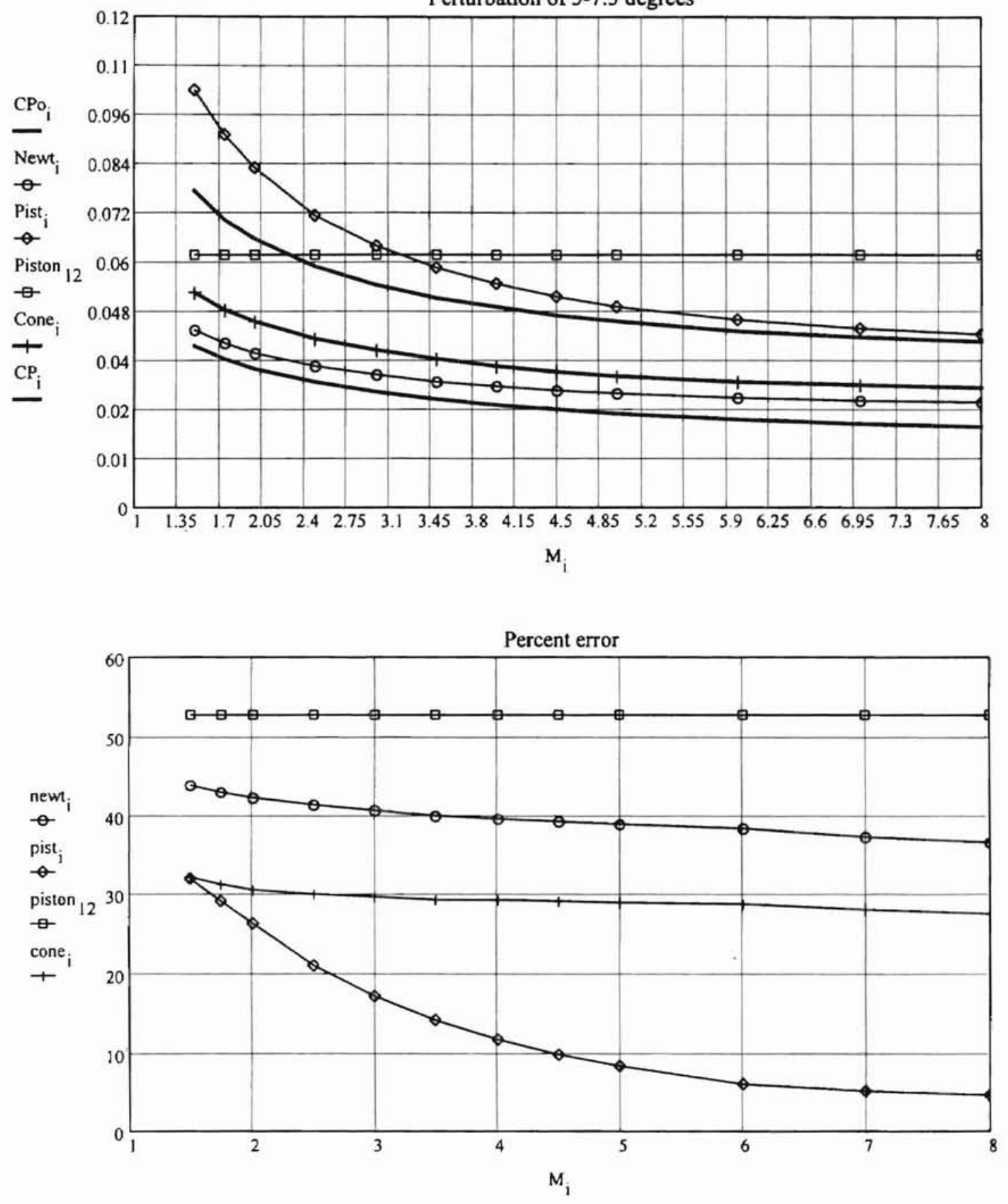


\section{Cone in Expansion}

Note, in the following charts, "Newt" is Newtonian, "Pist" is the P.S. method, "Piston" is straight piston, and "cone" is tangent cone. Also, "Cpo" is the mean flow pressure coefficient and " $\mathrm{Cp}$ " is the final exact pressure coefficient. Finally, a straight line represents the nearest value to the exact value or lowest percent error.
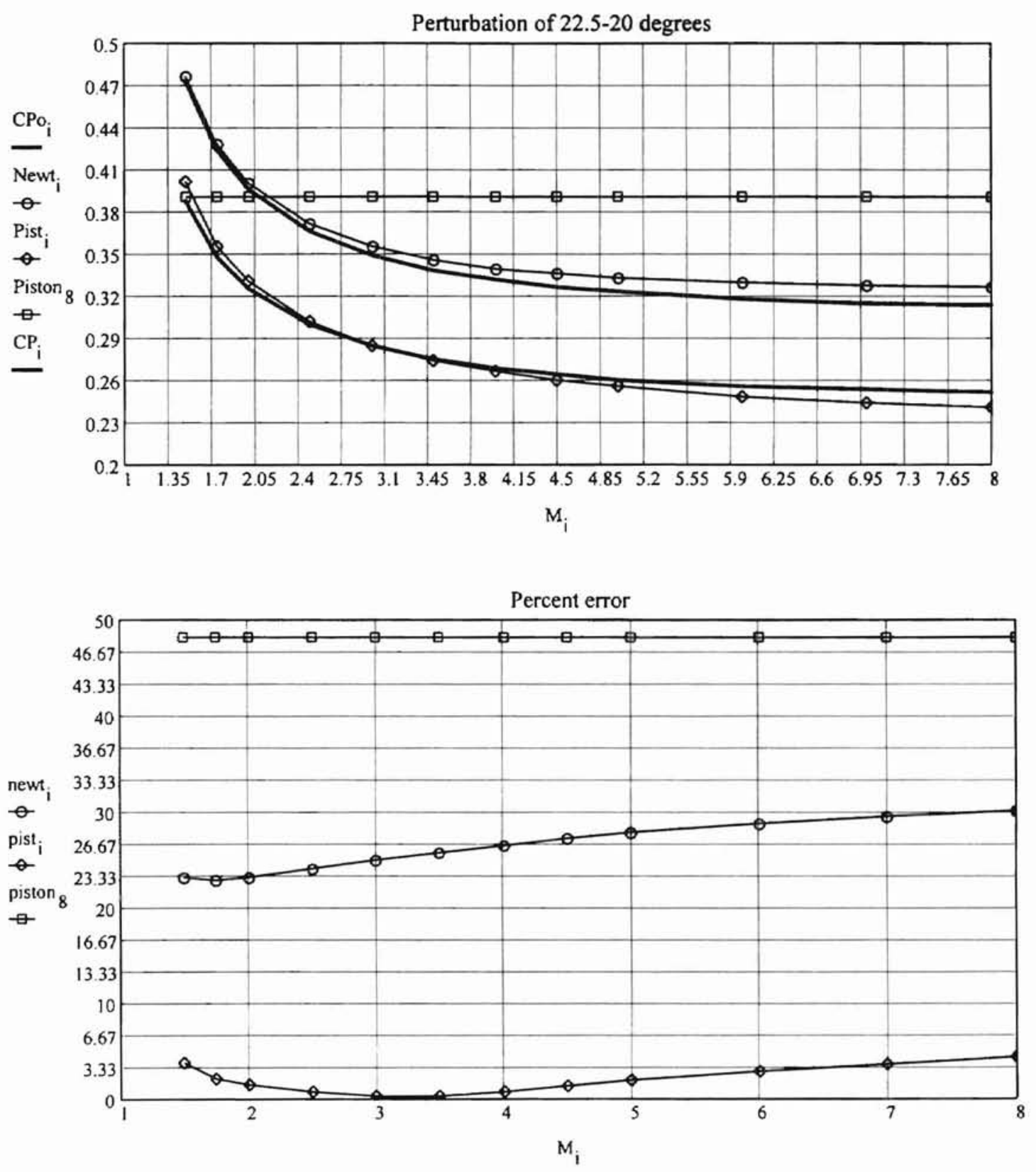
Perturbation of 15 to 12.5 degrees
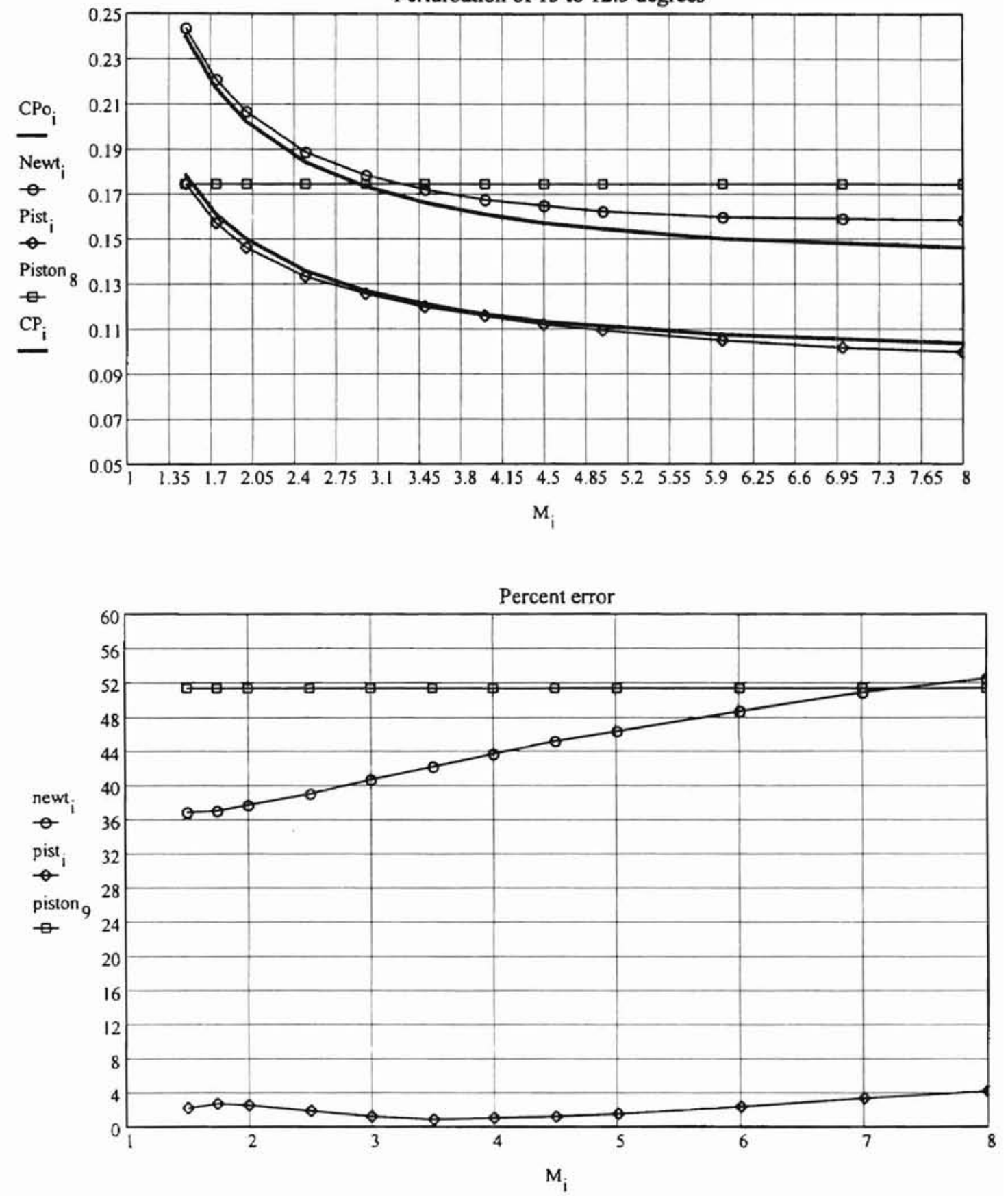

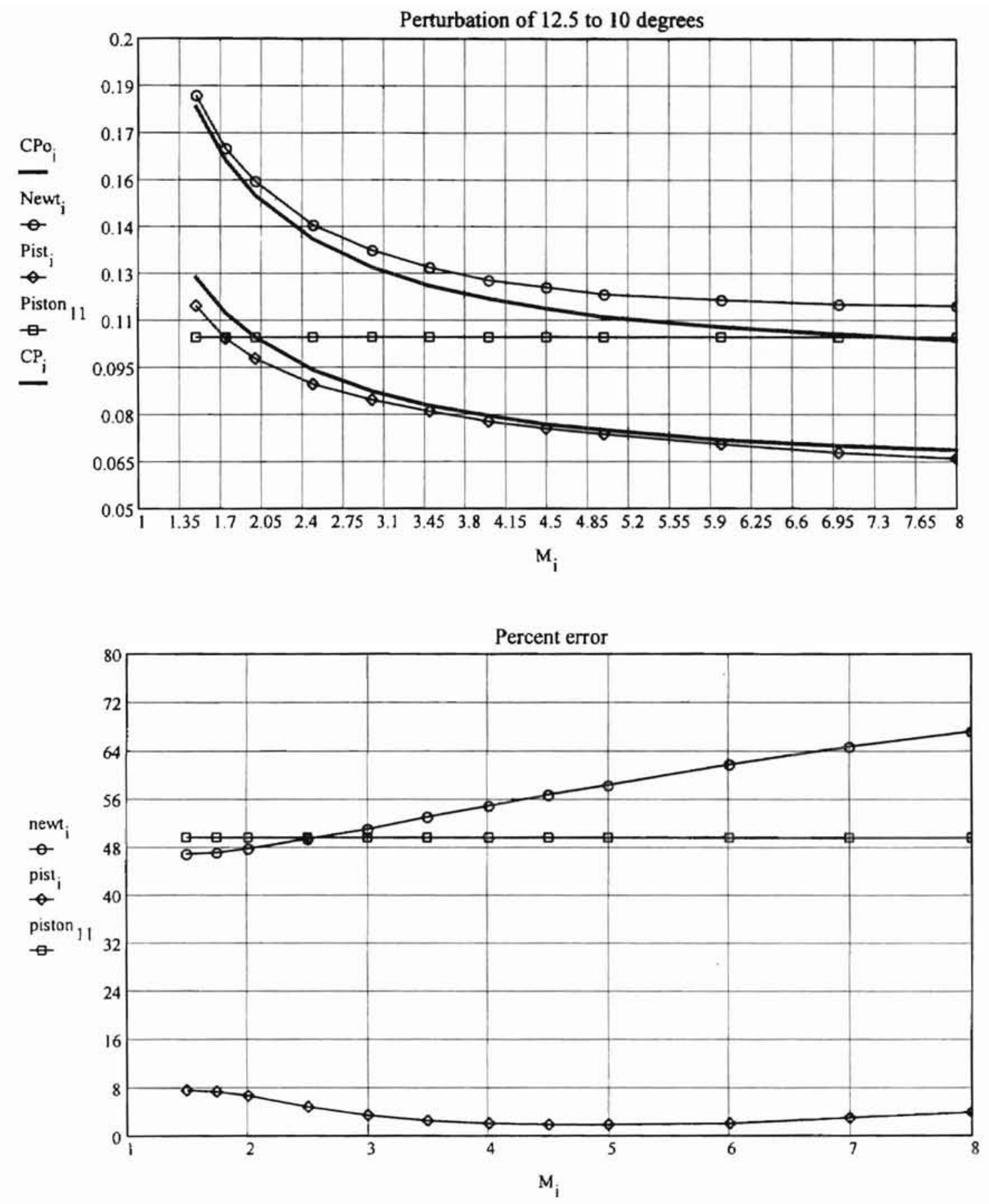

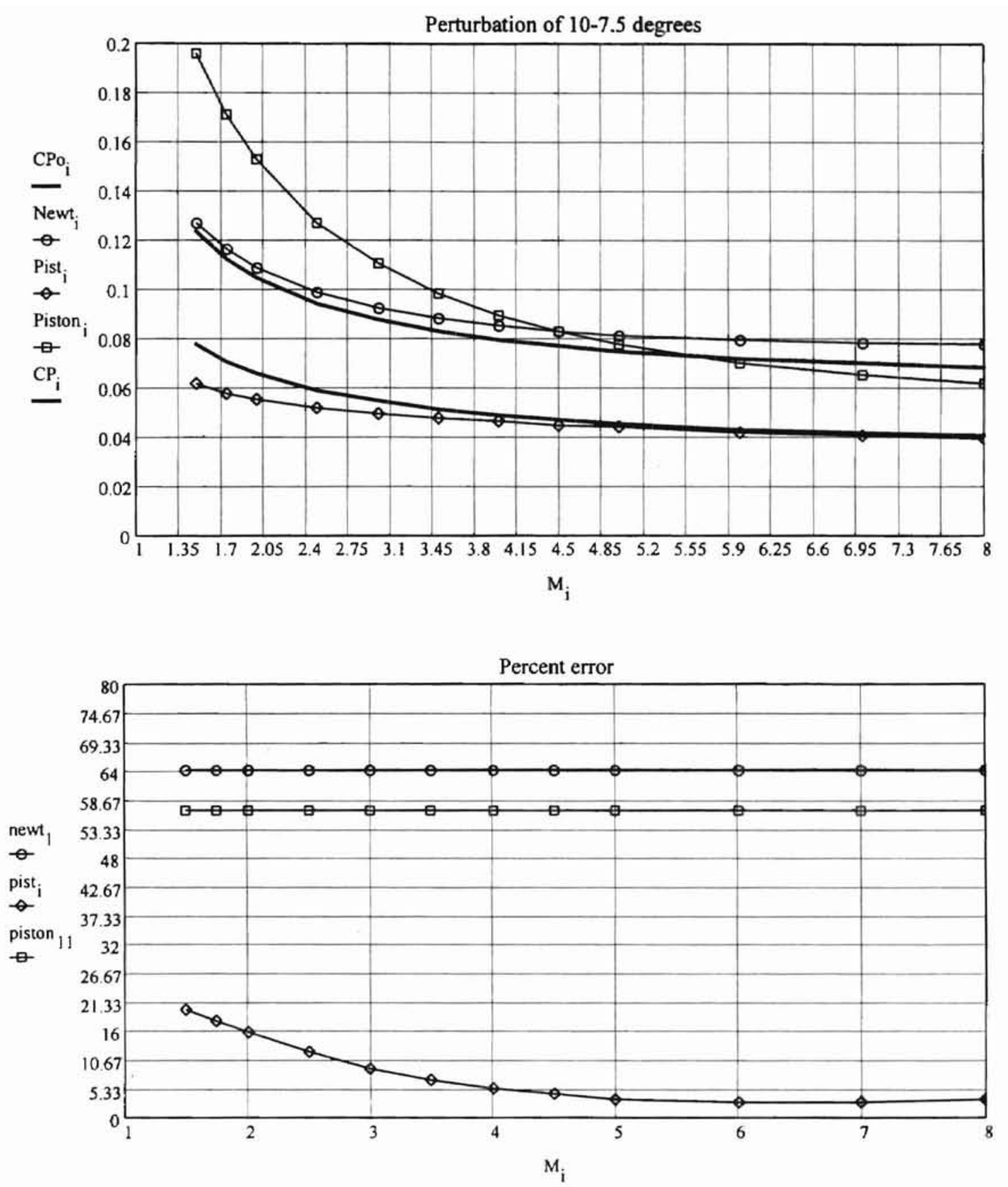
Perturbation of 7.5-5 degrees
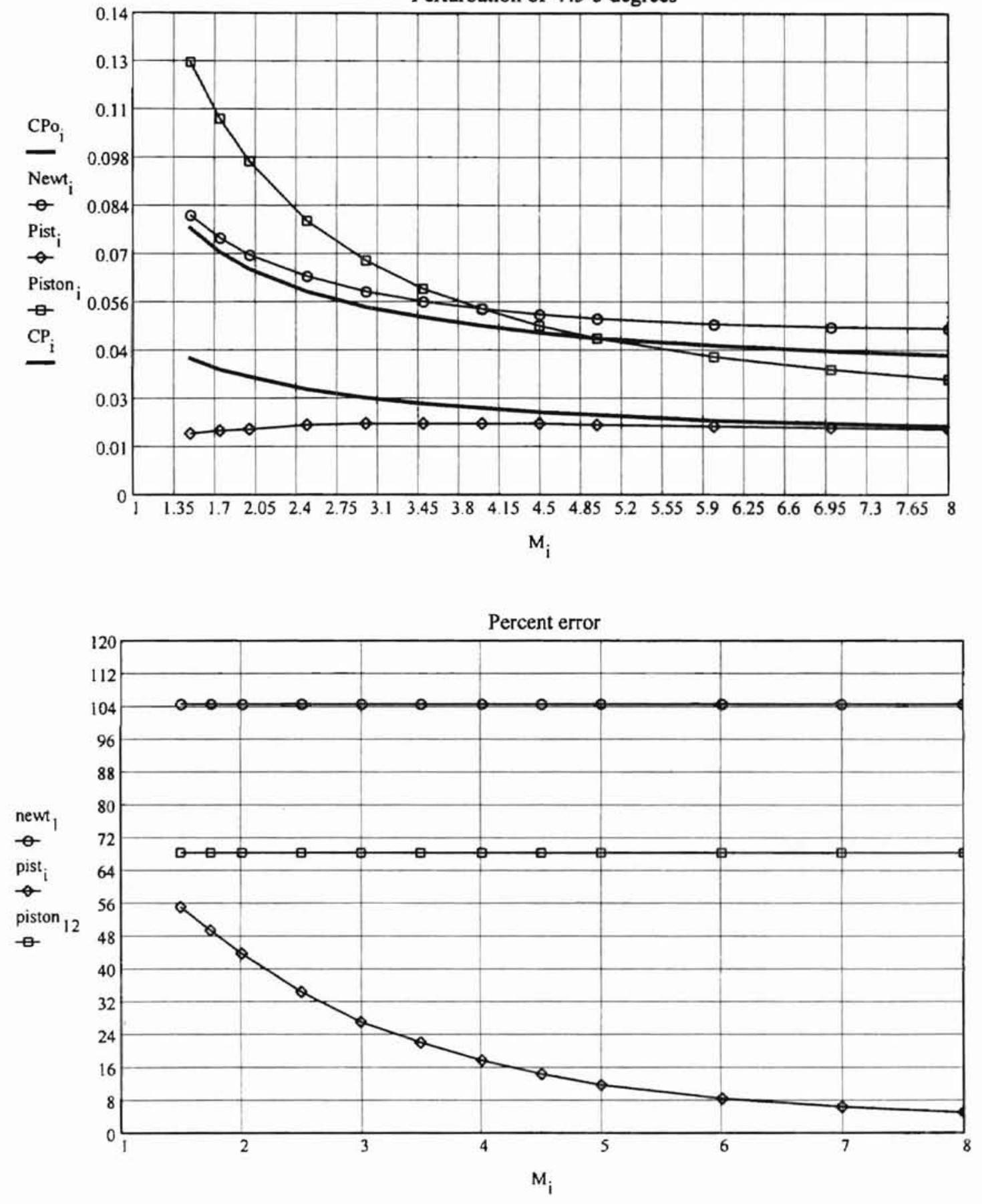


\section{APPENDIX B: CSWC DATA FILES}

CSWC dat file

\begin{tabular}{|c|c|c|c|c|c|}
\hline 1 & definition & felisa $\times 35$ & 1.2 & -9.2499 & \\
\hline 14 & 8 & & 2.9 & -9.7770 & \\
\hline Curves & & & 5 & -10.000 & \\
\hline 1 & 1 & & 7.1 & -9.7770 & \\
\hline 21 & & & 8.8 & -9.2499 & \\
\hline-5 & 0.0000 & & 10.3 & -8.4800 & \\
\hline-4.95 & 0.9987 & & 11.6 & -7.5127 & \\
\hline-4.75 & 2.2220 & & 12.8 & -6.2578 & \\
\hline-4.35 & 3.5465 & & 13.65 & -5.0177 & \\
\hline-3.65 & 5.0177 & & 14.35 & -3.5465 & \\
\hline-2.8 & 6.2578 & & 14.75 & -2.2220 & \\
\hline-1.6 & 7.5127 & & 14.95 & -0.9987 & \\
\hline-0.3 & 8.4800 & & 15 & 0.0000 & \\
\hline 1.2 & 9.2499 & & 3 & 1 & \\
\hline 2.9 & 9.7770 & & 15 & & \\
\hline 5 & 10.0000 & & 0 & 0.0000 & \\
\hline 7.1 & 9.7770 & & 1 & 0.2680 & \\
\hline 8.8 & 9.2499 & & 2.8 & 0.7503 & \\
\hline 10.3 & 8.4800 & & 4.5 & 1.2058 & \\
\hline 11.6 & 7.5127 & & 5.5 & 1.4737 & \\
\hline 12.8 & 6.2578 & & 5.95 & 1.5943 & \\
\hline 13.65 & 5.0177 & & 6.46 & 1.6700 & \\
\hline 14.35 & 3.5465 & & 6.95 & 1.6900 & \\
\hline 14.75 & 2.2220 & & 7.45 & 1.6000 & \\
\hline 14.95 & 0.9987 & & 7.85 & 1.4675 & \\
\hline 15 & 0.0000 & & 8.2 & 1.3100 & \\
\hline 2 & 1 & & 8.5 & 1.1750 & \\
\hline 21 & & & 8.9 & 0.9950 & \\
\hline-5 & 0.0000 & & 9.8 & 0.5900 & \\
\hline-4.95 & -0.9987 & & 11.11111 & 0 & 0.0000 \\
\hline-4.75 & -2.2220 & & 4 & 1 & \\
\hline-4.35 & -3.5465 & & 15 & & \\
\hline-3.65 & -5.0177 & & 0 & 0.0000 & \\
\hline-2.8 & -6.2578 & & 1 & -0.2680 & \\
\hline-1.6 & -7.5127 & & 2.8 & -0.7503 & \\
\hline-0.3 & -8.4800 & & 4.5 & -1.2058 & \\
\hline
\end{tabular}




\begin{tabular}{|c|c|c|c|}
\hline 5.5 & 0 & \multicolumn{2}{|l|}{-1.4737} \\
\hline 5.95 & 0 & \multicolumn{2}{|l|}{-1.5943} \\
\hline 6.46 & 0 & \multicolumn{2}{|l|}{-1.6700} \\
\hline 6.95 & 0 & \multicolumn{2}{|l|}{-1.6900} \\
\hline 7.45 & 0 & \multicolumn{2}{|l|}{-1.6000} \\
\hline 7.85 & 0 & \multicolumn{2}{|l|}{-1.4675} \\
\hline 8.2 & 0 & \multicolumn{2}{|l|}{-1.3100} \\
\hline 8.5 & 0 & \multicolumn{2}{|l|}{-1.1750} \\
\hline 8.9 & 0 & \multicolumn{2}{|l|}{-0.9950} \\
\hline 9.8 & 0 & \multicolumn{2}{|l|}{-0.5900} \\
\hline \multicolumn{2}{|c|}{11.11111} & \multirow[t]{3}{*}{0} & \multirow[t]{3}{*}{0.0000} \\
\hline 5 & 1 & & \\
\hline \multicolumn{2}{|c|}{19} & & \\
\hline \multicolumn{4}{|c|}{1.71590 .459850 .0000} \\
\hline \multirow{2}{*}{\multicolumn{2}{|c|}{$\begin{array}{l}1.746025415 \\
1.835486318\end{array}$}} & 0.4679 & 0.0042 \\
\hline & & 0.4916 & 0.0165 \\
\hline \multicolumn{2}{|c|}{1.981564487} & 0.5299 & 0.0356 \\
\hline \multicolumn{2}{|c|}{2.17982142} & 0.5811 & 0.0598 \\
\hline \multicolumn{2}{|c|}{2.42423319} & 0.6439 & 0.0863 \\
\hline \multicolumn{2}{|c|}{2.707373481} & 0.7168 & 0.1123 \\
\hline 3.0206 & 39234 & 0.7982 & 0.1348 \\
\hline 3.3545 & 12043 & 0.8862 & 0.1510 \\
\hline 3.6988 & 47369 & 0.9784 & 0.1590 \\
\hline 4.0431 & 82775 & 1.072 & 0.1576 \\
\hline 4.3770 & 55822 & 1.1638 & 0.1466 \\
\hline 4.6903 & 21962 & 1.2505 & 0.1273 \\
\hline 4.97346 & 62779 & 1.329 & 0.1016 \\
\hline $5.2178 ?$ & 75198 & 1.3965 & 0.0729 \\
\hline 5.41613 & 32882 & 1.4508 & 0.0448 \\
\hline 5.56221 & 1883 & 1.4905 & 0.0212 \\
\hline 5.65167 & 73671 & 1.5146 & 0.0055 \\
\hline 5.6818 & 1.5227 & 0.0000 & \\
\hline 6 & 1 & & \\
\hline 19 & & & \\
\hline 1.7159 & 0.45985 & 0.0000 & \\
\hline 1.74602 & 25415 & 0.4679 & -0.0042 \\
\hline 1.83548 & 86318 & 0.4916 & -0.0165 \\
\hline 1.98156 & 64487 & 0.5299 & -0.0356 \\
\hline 2.17982 & 2142 & 0.5811 & -0.0598 \\
\hline 2.42423 & 3319 & 0.6439 & -0.0863 \\
\hline 2.707 & 73481 & 0.7168 & -0.1123 \\
\hline 3.020 & 34 & 0.7982 & -0.1348 \\
\hline 3.354 & 120 & 0.8862 & -0.1510 \\
\hline 3.6988 & 47369 & 0.9784 & -0.1590 \\
\hline 4.04318 & 82775 & 1.072 & -0.1576 \\
\hline 4.37705 & 55822 & 1.1638 & -0.1466 \\
\hline 4.69032 & 21962 & 1.2505 & -0.1273 \\
\hline $4.9734 \mathrm{C}$ & 62779 & 1.329 & -0.1016 \\
\hline 5.2178 & 75198 & 1.3965 & -0.0729 \\
\hline 5.41613 & 32882 & 1.4508 & -0.0448 \\
\hline 5.5622 & 11883 & 1.4905 & -0.0212 \\
\hline $5.6516^{\circ}$ & 73671 & 1.5146 & -0.0055 \\
\hline 5.6818 & 1.5227 & 0.0000 & \\
\hline 7 & 1 & & \\
\hline
\end{tabular}

$\begin{array}{llll}2 & & & \\ 5.6818 & 1.5226 & 0.0000 & \\ 5.9394 & 2.4242 & 0.0000 & \\ 8 & 1 & & \\ 2 & & & \\ 5.9394 & 2.4242 & 0.0000 & \\ 7.5017 & 4.8597 & 0.0000 & \\ 9 & 1 & & \\ 19 & & & \\ 6.6024 & 4.8597 & 0.0000 & \\ 6.609231182 & 4.8597 & 0.0014 \\ 6.629517168 & 4.8597 & 0.0053 \\ 6.662641578 & 4.8597 & 0.0113 \\ 6.707597946 & 4.8597 & 0.0186 \\ 6.763020297 & 4.8597 & 0.0264 \\ 6.827224656 & 4.8597 & 0.0337 \\ 6.898260207 & 4.8597 & 0.0397 \\ 6.973968575 & 4.8597 & 0.0436 \\ 7.052049403 & 4.8597 & 0.0450 \\ 7.13013025 & 4.8597 & 0.0436 \\ 7.205838672 & 4.8597 & 0.0397 \\ 7.276874311 & 4.8597 & 0.0337 \\ 7.341078789 & 4.8597 & 0.0264 \\ 7.396501287 & 4.8597 & 0.0186 \\ 7.441457826 & 4.8597 & 0.0113 \\ 7.474582424 & 4.8597 & 0.0053 \\ 7.49486861 & 4.8597 & 0.0014 \\ 7.5017 & 4.8597 & 0.0000 & \\ 10 & 1 & & \\ 19 & & & \\ 6.6024 & 4.8597 & 0.0000 & \\ 6.609231182 & 4.8597 & -0.0014 \\ 6.629517168 & 4.8597 & -0.0053 \\ 6.662641578 & 4.8597 & -0.0113 \\ 6.707597946 & 4.8597 & -0.0186 \\ 6.763020297 & 4.8597 & -0.0264 \\ 6.827224656 & 4.8597 & -0.0337 \\ 6.898260207 & 4.8597 & -0.0397 \\ 6.973968575 & 4.8597 & -0.0436 \\ 7.052049403 & 4.8597 & -0.0450 \\ 7.13013025 & 4.8597 & -0.0436 \\ 7.205838672 & 4.8597 & -0.0397 \\ 7.276874311 & 4.8597 & -0.0337 \\ 7.341078789 & 4.8597 & -0.0264 \\ 7.396501287 & 4.8597 & -0.0186 \\ 7.441457826 & 4.8597 & -0.0113 \\ 7.474582424 & 4.8597 & -0.0053 \\ 7.49486861 & 4.8597 & -0.0014 \\ 7.5017 & 4.8597 & 0.0000 & \\ 11 & 1 & & \\ 2 & & & \\ 6.6024 & 4.8597 & 0.0000 & \\ 3.8975 & 2.4242 & 0.0000 & \\ 12 & 1 & & \\ & & & \\ & 1 & \end{array}$




\begin{tabular}{|c|c|c|c|c|c|}
\hline 19 & & & -1.6 & 0 & 7.5127 \\
\hline $3.8975 \quad 2.4242$ & 0.0000 & & -0.3 & 0 & 8.4800 \\
\hline 3.913010498 & 2.4242 & 0.0031 & 1.2 & 0 & 9.2499 \\
\hline 3.959070716 & 2.4242 & 0.0120 & 2.9 & 0 & 9.7770 \\
\hline 4.034281138 & 2.4242 & 0.0255 & 5 & 0 & 10.0000 \\
\hline 4.136356539 & 2.4242 & 0.0422 & 7.1 & 0 & 9.7770 \\
\hline 4.262195413 & 2.4242 & 0.0599 & 8.8 & 0 & 9.2499 \\
\hline 4.407974218 & 2.4242 & 0.0766 & 10.3 & 0 & 8.4800 \\
\hline 4.569263545 & 2.4242 & 0.0902 & 11.6 & 0 & 7.5127 \\
\hline 4.741162707 & 2.4242 & 0.0990 & 12.8 & 0 & 6.2578 \\
\hline 4.918448645 & 2.4242 & 0.1021 & 13.65 & 0 & 5.0177 \\
\hline 5.095734625 & 2.4242 & 0.0990 & 14.35 & 0 & 3.5465 \\
\hline 5.26763391 & 2.4242 & 0.0902 & 14.75 & 0 & 2.2220 \\
\hline 5.428923436 & 2.4242 & 0.0766 & 14.95 & 0 & 0.9987 \\
\hline 5.574702511 & 2.4242 & 0.0599 & 15 & 0 & 0.0000 \\
\hline 5.70054172 & 2.4242 & 0.0422 & -5 & 0 & 0.0000 \\
\hline 5.802617507 & 2.4242 & 0.0255 & -4.95 & 0 & -0.9987 \\
\hline 5.877828358 & 2.4242 & 0.0119 & -4.75 & 0 & -2.2220 \\
\hline 5.923889031 & 2.4242 & 0.0031 & -4.35 & 0 & -3.5465 \\
\hline $5.9394 \quad 2.4242$ & 0.0000 & & -3.65 & 0 & -5.0177 \\
\hline $13 \quad 1$ & & & -2.8 & 0 & -6.2578 \\
\hline 19 & & & -1.6 & 0 & -7.5127 \\
\hline $3.8975 \quad 2.4242$ & 0.0000 & & -0.3 & 0 & -8.4800 \\
\hline 3.913010498 & 2.4242 & -0.0031 & 1.2 & 0 & -9.2499 \\
\hline 3.959070716 & 2.4242 & -0.0120 & 2.9 & 0 & -9.7770 \\
\hline 4.034281138 & 2.4242 & -0.0255 & 5 & 0 & -10.0000 \\
\hline 4.136356539 & 2.4242 & -0.0422 & 7.1 & 0 & -9.7770 \\
\hline 4.262195413 & 2.4242 & -0.0599 & 8.8 & 0 & -9.2499 \\
\hline 4.407974218 & 2.4242 & -0.0766 & 10.3 & 0 & -8.4800 \\
\hline 4.569263545 & 2.4242 & -0.0902 & 11.6 & 0 & -7.5127 \\
\hline 4.741162707 & 2.4242 & -0.0990 & 12.8 & 0 & -6.2578 \\
\hline 4.918448645 & 2.4242 & -0.1021 & 13.65 & 0 & -5.0177 \\
\hline 5.095734625 & 2.4242 & -0.0990 & 14.35 & 0 & -3.5465 \\
\hline 5.26763391 & 2.4242 & -0.0902 & 14.75 & 0 & -2.2220 \\
\hline 5.428923436 & 2.4242 & -0.0766 & 14.95 & 0 & -0.9987 \\
\hline 5.574702511 & 2.4242 & -0.0599 & 15 & 0 & 0.0000 \\
\hline 5.70054172 & 2.4242 & -0.0422 & 2 & 1 & \\
\hline 5.802617507 & 2.4242 & -0.0255 & 21 & 21 & \\
\hline 5.877828358 & 2.4242 & -0.0119 & -5 & 0 & 0.0000 \\
\hline 5.923889031 & 2.4242 & -0.0031 & -4.95 & 0 & 0.9987 \\
\hline $5.9394 \quad 2.4242$ & 0.0000 & & -4.75 & 0 & 2.2220 \\
\hline $14 \quad 1$ & & & -4.35 & 0 & 3.5465 \\
\hline 2 & & & -3.65 & 0 & 5.0177 \\
\hline $1.7159 \quad 0.4598$ & 0.0000 & & -2.8 & 0 & 6.2578 \\
\hline $3.8975 \quad 2.4242$ & 0.0000 & & -1.6 & 0 & 7.5127 \\
\hline Surfaces & & & -0.3 & 0 & 8.4800 \\
\hline 11 & & & 1.2 & 0 & 9.2499 \\
\hline 21 & & & 2.9 & 0 & 9.7770 \\
\hline-5 & 0.0000 & & 5 & 0 & 10.0000 \\
\hline-4.95 & 0.9987 & & 7.1 & 0 & 9.7770 \\
\hline-4.75 & 2.2220 & & 8.8 & 0 & 9.2499 \\
\hline-4.35 & 3.5465 & & 10.3 & 0 & 8.4800 \\
\hline-3.65 & 5.0177 & & 11.6 & 0 & 7.5127 \\
\hline-2.8 & 6.2578 & & 12.8 & 0 & 6.2578 \\
\hline
\end{tabular}




\begin{tabular}{|c|c|c|}
\hline 13.65 & 5.0177 & \\
\hline 14.35 & 3.5465 & \\
\hline 14.75 & 2.2220 & \\
\hline 14.95 & 0.9987 & \\
\hline 15 & 0.0000 & \\
\hline-5 & $\begin{array}{ll}0 & 0.0000\end{array}$ & \\
\hline-4.95 & 0.156238669 & 0.9865 \\
\hline-4.75 & 0.347604694 & 2.1947 \\
\hline-4.35 & 0.554790787 & 3.5028 \\
\hline-3.65 & 0.784943468 & 4.9559 \\
\hline-2.8 & 0.978934015 & 6.1808 \\
\hline-1.6 & 1.175237336 & 7.4202 \\
\hline-0.3 & 1.326559463 & 8.3756 \\
\hline 1.2 & 1.44699645 & 9.1360 \\
\hline 2.9 & 1.529460651 & 9.6566 \\
\hline 5 & 1.56434334 & 9.8769 \\
\hline 7.1 & 1.529460651 & 9.6566 \\
\hline 8.8 & 1.44699645 & 9.1360 \\
\hline 10.3 & 1.326559463 & 8.3756 \\
\hline 11.6 & 1.175237336 & 7.4202 \\
\hline 12.8 & 0.978934015 & 6.1808 \\
\hline 13.65 & 0.784943468 & 4.9559 \\
\hline 14.35 & 0.554790787 & 3.5028 \\
\hline 14.75 & 0.347604694 & 2.1947 \\
\hline 14.95 & 0.156238669 & 0.9865 \\
\hline 15 & $\begin{array}{ll}0 & 0.0000\end{array}$ & \\
\hline-5 & $\begin{array}{ll}0 & 0.0000\end{array}$ & \\
\hline-4.95 & 0.308630229 & 0.9499 \\
\hline-4.75 & 0.68665022 & 2.1133 \\
\hline-4.35 & 1.095920807 & 3.3729 \\
\hline-3.65 & 1.550559056 & 4.7721 \\
\hline-2.8 & 1.933763466 & 5.9515 \\
\hline-1.6 & 2.321536477 & 7.1450 \\
\hline-0.3 & 2.620454684 & 8.0649 \\
\hline 1.2 & 2.858363104 & 8.7971 \\
\hline 2.9 & 3.021260969 & 9.2985 \\
\hline 5 & 3.09016742 & 9.5106 \\
\hline 7.1 & 3.021260969 & 9.2985 \\
\hline 8.8 & 63104 & 8.7971 \\
\hline 10.3 & 2.620454684 & 8.0649 \\
\hline 11.6 & 2.321536477 & 7.1450 \\
\hline 12.8 & 1.933763466 & 5.9515 \\
\hline 13.65 & 1.550559056 & 4.7721 \\
\hline 14.35 & 1.095920807 & 3.3729 \\
\hline 14.75 & 0.68665022 & 2.1133 \\
\hline 14.95 & 0.308630229 & 0.9499 \\
\hline 15 & $\begin{array}{ll}0 & 0.0000\end{array}$ & \\
\hline-5 & 0.0000 & \\
\hline-4.95 & 0.453422302 & 0.8899 \\
\hline-4.75 & 1.008788168 & 1.9799 \\
\hline-4.35 & 1.610065665 & 3.1599 \\
\hline-3.65 & 2.277994798 & 4.4708 \\
\hline-2.8 & 2.840977323 & 5.5757 \\
\hline-1.6 & 3.410671782 & 6.6938 \\
\hline
\end{tabular}

\begin{tabular}{|c|c|c|}
\hline-0.3 & 3.849825723 & 7.5557 \\
\hline 1.2 & 4.199347492 & 8.2417 \\
\hline 2.9 & 4.43866794 & 8.7114 \\
\hline 5 & 4.539901451 & 8.9101 \\
\hline 7.1 & 4.43866794 & 8.7114 \\
\hline 8.8 & 4.199347492 & 8.2417 \\
\hline 10.3 & 3.849825723 & 7.5557 \\
\hline 11.6 & 3.410671782 & 6.6938 \\
\hline 12.8 & 2.840977323 & 5.5757 \\
\hline 13.65 & 2.277994798 & 4.4708 \\
\hline 14.35 & 1.610065665 & 3.1599 \\
\hline 14.75 & 1.008788168 & 1.9799 \\
\hline 14.95 & 0.453422302 & 0.8899 \\
\hline 15 & 0.0000 & \\
\hline-5 & 0.0000 & \\
\hline-4.95 & 0.587049632 & 0.8080 \\
\hline-4.75 & 1.306086445 & 1.7977 \\
\hline-4.35 & 2.084565429 & 2.8692 \\
\hline-3.65 & 2.949338842 & 4.0594 \\
\hline-2.8 & 3.678237008 & 5.0627 \\
\hline-1.6 & 4.41582517 & 6.0779 \\
\hline-0.3 & 4.984401436 & 6.8604 \\
\hline 1.2 & 5.436930181 & 7.4833 \\
\hline 2.9 & 5.74678036 & 7.9098 \\
\hline 5 & 5.877848229 & 8.0902 \\
\hline 7.1 & 5.74678036 & 7.9098 \\
\hline 8.8 & 5.436930181 & 7.4833 \\
\hline 10.3 & 4.984401436 & 6.8604 \\
\hline 11.6 & 4.41582517 & 6.0779 \\
\hline 12.8 & 3.678237008 & 5.0627 \\
\hline 13.65 & 2.949338842 & 4.0594 \\
\hline 14.35 & 2.084565429 & 2.8692 \\
\hline 14.75 & 1.306086445 & 1.7977 \\
\hline 14.95 & 0.587049632 & 0.8080 \\
\hline 15 & $\begin{array}{ll}0 & 0.0000\end{array}$ & \\
\hline-5 & $\begin{array}{ll}0 & 0.0000\end{array}$ & \\
\hline-4.95 & 0.706221876 & 0.7062 \\
\hline-4.75 & 1.571224594 & 1.5712 \\
\hline-4.35 & 2.507736361 & 2.5077 \\
\hline-3.65 & 3.548060498 & 3.5481 \\
\hline-2.8 & 4.424926442 & 4.4249 \\
\hline-1.6 & 5.31224647 & 5.3123 \\
\hline-0.3 & 5.996244849 & 5.9963 \\
\hline 1.2 & 6.540637831 & 6.5406 \\
\hline 2.9 & 6.913388213 & 6.9134 \\
\hline 5 & 7.071063121 & 7.0711 \\
\hline 7.1 & 6.913388213 & 6.9134 \\
\hline 8.8 & 6.540637831 & 6.5406 \\
\hline 10.3 & 5.996244849 & 5.9963 \\
\hline 11.6 & 5.31224647 & 5.3123 \\
\hline 12.8 & 4.424926442 & 4.4249 \\
\hline 13.65 & 3.548060498 & 3.5481 \\
\hline 14.35 & 2.507736361 & 2.5077 \\
\hline 14.75 & 1.571224594 & 1.5712 \\
\hline
\end{tabular}




\begin{tabular}{|c|c|c|c|c|c|}
\hline 14.95 & 0.706221876 & 0.7062 & 5 & 9.510561883 & 3.0902 \\
\hline 15 & $\begin{array}{ll}0 & 0.0000\end{array}$ & & 7.1 & 9.298489534 & 3.0213 \\
\hline-5 & $\begin{array}{ll}0 & 0.0000\end{array}$ & & 8.8 & 8.79714122 & 2.8584 \\
\hline-4.95 & 0.808004623 & 0.5871 & 10.3 & 8.064934046 & 2.6205 \\
\hline-4.75 & 1.797674043 & 1.3061 & 11.6 & 7.144957968 & 2.3215 \\
\hline-4.35 & 2.869158605 & 2.0846 & 12.8 & 5.951514792 & 1.9338 \\
\hline-3.65 & 4.059417277 & 2.9493 & 13.65 & 4.77213233 & 1.5506 \\
\hline-2.8 & 5.062659686 & 3.6782 & 14.35 & 3.372899017 & 1.0959 \\
\hline-1.6 & 6.077862852 & 4.4158 & 14.75 & 2.113293076 & 0.6867 \\
\hline-0.3 & 6.860441064 & 4.9844 & 14.95 & 0.949866624 & 0.3086 \\
\hline 1.2 & 7.483293542 & 5.4369 & 15 & $\begin{array}{ll}0 & 0.0000\end{array}$ & \\
\hline 2.9 & 7.909765791 & 5.7468 & -5 & $\begin{array}{ll}0 & 0.0000\end{array}$ & \\
\hline 5 & 8.090165265 & 5.8779 & -4.95 & 0.986452771 & 0.1562 \\
\hline 7.1 & 7.909765791 & 5.7468 & -4.75 & 2.194691084 & 0.3476 \\
\hline 8.8 & 7.483293542 & 5.4369 & -4.35 & 3.502813444 & 0.5548 \\
\hline 10.3 & 6.860441064 & 4.9844 & -3.65 & 4.955941225 & 0.7850 \\
\hline 11.6 & 6.077862852 & 4.4158 & -2.8 & 6.180750128 & 0.9789 \\
\hline 12.8 & 5.062659686 & 3.6782 & -1.6 & 7.420161323 & 1.1752 \\
\hline 13.65 & 4.059417277 & 2.9493 & -0.3 & 8.37557225 & 1.3266 \\
\hline 14.35 & 2.869158605 & 2.0846 & 1.2 & 9.13598195 & 1.4470 \\
\hline 14.75 & 1.797674043 & 1.3061 & 2.9 & 9.656640768 & 1.5295 \\
\hline 14.95 & 0.808004623 & 0.5871 & 5 & 9.876881538 & 1.5644 \\
\hline 15 & $\begin{array}{ll}0 & 0.0000\end{array}$ & & 7.1 & 9.656640768 & 1.5295 \\
\hline-5 & $\begin{array}{ll}0 & 0.0000\end{array}$ & & 8.8 & 9.13598195 & 1.4470 \\
\hline-4.95 & 0.889891648 & 0.4534 & 10.3 & 8.37557225 & 1.3266 \\
\hline-4.75 & 1.979858867 & 1.0088 & 11.6 & 7.420161323 & 1.1752 \\
\hline-4.35 & 3.159932761 & 1.6101 & 12.8 & 6.180750128 & 0.9789 \\
\hline-3.65 & 4.470817898 & 2.2780 & 13.65 & 4.955941225 & 0.7850 \\
\hline-2.8 & 5.575733657 & 2.8410 & 14.35 & 3.502813444 & 0.5548 \\
\hline-1.6 & 6.693822332 & 3.4107 & 14.75 & 2.194691084 & 0.3476 \\
\hline-0.3 & 7.555710735 & 3.8498 & 14.95 & 0.986452771 & 0.1562 \\
\hline 1.2 & 8.241686041 & 4.1994 & 15 & $\begin{array}{ll}0 & 0.0000\end{array}$ & \\
\hline 2.9 & 8.711379013 & 4.4387 & -5 & $\begin{array}{ll}0 & 0.0000\end{array}$ & \\
\hline 5 & 8.910061025 & 4.5399 & -4.95 & 0.998749218 & 0.0000 \\
\hline 7.1 & 8.711379013 & 4.4387 & -4.75 & 2.222048604 & 0.0000 \\
\hline 8.8 & 8.241686041 & 4.1994 & -4.35 & 3.546477125 & 0.0000 \\
\hline 10.3 & 7.555710735 & 3.8498 & -3.65 & 5.017718605 & 0.0000 \\
\hline 11.6 & 6.693822332 & 3.4107 & -2.8 & 6.257795139 & 0.0000 \\
\hline 12.8 & 5.575733657 & 2.8410 & -1.6 & 7.512655988 & 0.0000 \\
\hline 13.65 & 4.470817898 & 2.2780 & -0.3 & 8.479976415 & 0.0000 \\
\hline 14.35 & 3.159932761 & 1.6101 & 1.2 & 9.249864864 & 0.0000 \\
\hline 14.75 & 1.979858867 & 1.0088 & 2.9 & 9.777013859 & 0.0000 \\
\hline 14.95 & 0.889891648 & 0.4534 & 5 & $\begin{array}{ll}10 & 0.0000\end{array}$ & \\
\hline 15 & 0.0000 & & 7.1 & 9.777013859 & 0.0000 \\
\hline-5 & $\begin{array}{ll}0 & 0.0000\end{array}$ & & 8.8 & 9.249864864 & 0.0000 \\
\hline-4.95 & 0.949866624 & 0.3086 & 10.3 & 8.479976415 & 0.0000 \\
\hline-4.75 & 2.113293076 & 0.6867 & 11.6 & 7.512655988 & 0.0000 \\
\hline-4.35 & 3.372899017 & 1.0959 & 12.8 & 6.257795139 & 0.0000 \\
\hline-3.65 & 4.77213233 & 1.5506 & 13.65 & 5.017718605 & 0.0000 \\
\hline-2.8 & 5.951514792 & 1.9338 & 14.35 & 3.546477125 & 0.0000 \\
\hline-1.6 & 7.144957968 & 2.3215 & 14.75 & 2.222048604 & 0.0000 \\
\hline-0.3 & 8.064934046 & 2.6205 & 14.95 & 0.998749218 & 0.0000 \\
\hline 1.2 & 8.79714122 & 2.8584 & 15 & $\begin{array}{ll}0 & 0.0000\end{array}$ & \\
\hline 2.9 & 9.298489534 & 3.0213 & -5 & 0.0000 & \\
\hline
\end{tabular}




\begin{tabular}{|c|c|c|}
\hline-4.95 & 0.986453186 & -0.1562 \\
\hline-4.75 & 2.194692006 & -0.3476 \\
\hline-4.35 & 3.502814917 & -0.5548 \\
\hline-3.65 & 4.955943308 & -0.7849 \\
\hline-2.8 & 6.180752725 & -0.9789 \\
\hline-1.6 & 7.420164442 & -1.1752 \\
\hline-0.3 & 8.37557577 & -1.3265 \\
\hline 1.2 & 9.13598579 & -1.4470 \\
\hline 2.9 & 9.656644827 & -1.5294 \\
\hline 5 & 9.876885689 & -1.5643 \\
\hline 7.1 & 9.656644827 & -1.5294 \\
\hline 8.8 & 9.13598579 & -1.4470 \\
\hline 10.3 & 8.37557577 & -1.3265 \\
\hline 11.6 & 64442 & -1.1752 \\
\hline 12.8 & 2725 & -0.9789 \\
\hline 13.65 & 3308 & -0.7849 \\
\hline 14.35 & 4917 & -0.5548 \\
\hline 14.75 & 92006 & -0.3476 \\
\hline 14.95 & 153186 & -0.1562 \\
\hline 15 & 0.0000 & \\
\hline-5 & 0.0000 & \\
\hline-4.95 & 0.949867443 & -0.3086 \\
\hline-4.75 & 2.113294898 & -0.6866 \\
\hline-4.35 & 3.372901925 & -1.0959 \\
\hline-3.65 & 4.772136445 & -1.5506 \\
\hline-2.8 & 519923 & -1.9338 \\
\hline-1.6 & 64129 & -2.3215 \\
\hline-0.3 & 8.06 & -2.6204 \\
\hline 1.2 & 8.797 & -2.8584 \\
\hline 2.9 & 9.298 & -3.0212 \\
\hline 5 & 9.510 & -3.0902 \\
\hline 7.1 & 9.298497551 & -3.0212 \\
\hline 8.8 & 8.797148805 & -2.8 \\
\hline 10.3 & 8.064941 & -2.6204 \\
\hline 11.6 & 7.144964129 & -2.3215 \\
\hline 12.8 & 5.951519923 & -1.9338 \\
\hline 13.65 & 4.772136445 & -1.5506 \\
\hline 14.35 & 3.372901925 & -1.0959 \\
\hline 14.75 & 2.113294898 & -0.6866 \\
\hline 14.95 & 0.949867443 & -0.3086 \\
\hline 15 & $\begin{array}{ll}0 & 0.0000\end{array}$ & \\
\hline-5 & $\begin{array}{ll}0 & 0.0000\end{array}$ & \\
\hline-4.95 & 0.889892851 & -0.4534 \\
\hline-4.75 & 1.979861544 & -1.0088 \\
\hline-4.35 & 3.159937034 & -1.6101 \\
\hline-3.65 & 4.470823943 & -2.2780 \\
\hline-2.8 & 5.575741196 & -2.8410 \\
\hline-1.6 & 6.6938313 & -3.4107 \\
\hline-0.3 & 7.5557209 & -3.8498 \\
\hline 1.2 & 8.241697185 & -4.1993 \\
\hline 2.9 & 8.711390791 & -4.4387 \\
\hline 5 & $8.9 i 0073072$ & -4.5399 \\
\hline 7.1 & 8.711390791 & -4.4387 \\
\hline 8.8 & 8.241697185 & -4.1993 \\
\hline
\end{tabular}

\begin{tabular}{|c|c|c|}
\hline 10.3 & 7.555720951 & -3.8498 \\
\hline 11.6 & 6.693831382 & -3.4107 \\
\hline 12.8 & 5.575741196 & -2.8410 \\
\hline 13.65 & 4.470823943 & -2.2780 \\
\hline 14.35 & 3.159937034 & -1.6101 \\
\hline 14.75 & 1.979861544 & -1.0088 \\
\hline 14.95 & 0.889892851 & -0.4534 \\
\hline 15 & $\begin{array}{ll}0 & 0.0000\end{array}$ & \\
\hline-5 & 0.0000 & \\
\hline-4.95 & 0.808006181 & -0.5870 \\
\hline-4.75 & 1.797677509 & -1.3061 \\
\hline-4.35 & 2.869164137 & -2.0846 \\
\hline-3.65 & 4.059425103 & -2.9493 \\
\hline-2.8 & 5.062669447 & -3.6782 \\
\hline-1.6 & 6.07787457 & -4.4158 \\
\hline-0.3 & 6.86045429 & -4.9844 \\
\hline 1.2 & 7.48330797 & -5.4369 \\
\hline 2.9 & 7.909781041 & -5.7468 \\
\hline 5 & 8.090180862 & -5.8778 \\
\hline 7.1 & 7.909781041 & -5.7468 \\
\hline 8.8 & 7.48330797 & -5.4369 \\
\hline 10.3 & 6.86045429 & -4.9844 \\
\hline 11.6 & 6.07787457 & -4.4158 \\
\hline 12.8 & 5.062669447 & -3.6782 \\
\hline 13.65 & 4.059425103 & -2.9493 \\
\hline 14.35 & 2.869164137 & -2.0846 \\
\hline 14.75 & 1.797677509 & -1.3061 \\
\hline 14.95 & 0.808006181 & -0.5870 \\
\hline 15 & $\begin{array}{ll}0 & 0.0000\end{array}$ & \\
\hline-5 & $\begin{array}{ll}0 & 0.0000\end{array}$ & \\
\hline-4.95 & 0.70622375 & -0.7062 \\
\hline-4.75 & 1.571228763 & -1.5712 \\
\hline-4.35 & 2.507743015 & -2.5077 \\
\hline-3.65 & 3.548069913 & -3.5481 \\
\hline-2.8 & 4.424938184 & -4.4249 \\
\hline-1.6 & 5.312260567 & -5.3122 \\
\hline-0.3 & 5.996260761 & -5.9962 \\
\hline 1.2 & 6.540655187 & -6.5406 \\
\hline 2.9 & 6.913406558 & -6.9134 \\
\hline 5 & 7.071081885 & -7.0711 \\
\hline 7.1 & 6.913406558 & -6.9134 \\
\hline 8.8 & 6.540655187 & -6.5406 \\
\hline 10.3 & 5.996260761 & -5.9962 \\
\hline 11.6 & 5.312260567 & -5.3122 \\
\hline 12.8 & 4.424938184 & -4.4249 \\
\hline 13.65 & 3.548069913 & -3.5481 \\
\hline 14.35 & 2.507743015 & -2.5077 \\
\hline 14.75 & 1.571228763 & -1.5712 \\
\hline 14.95 & 0.70622375 & -0.7062 \\
\hline 15 & 0.0000 & \\
\hline-5 & 0.0000 & \\
\hline-4.95 & 0.587051776 & -0.8080 \\
\hline-4.75 & 1.306091216 & -1.7977 \\
\hline-4.35 & 2.084573043 & -2.8692 \\
\hline
\end{tabular}




\begin{tabular}{|c|c|c|c|c|c|c|}
\hline-3.65 & 2.949349614 & -4.0594 & 13.65 & \multicolumn{2}{|c|}{1.550571719} & -4.7721 \\
\hline-2.8 & 3.678250442 & -5.0627 & 14.35 & \multicolumn{2}{|c|}{1.095929757} & -3.3729 \\
\hline-1.6 & 4.415841298 & -6.0779 & 14.75 & \multicolumn{2}{|c|}{0.686655828} & -2.1133 \\
\hline-0.3 & 4.98441964 & -6.8604 & 14.95 & \multicolumn{2}{|c|}{0.30863275} & -0.9499 \\
\hline 1.2 & 5.436950039 & -7.4833 & 15 & 0 & 0.0000 & \\
\hline 2.9 & 5.746801349 & -7.9098 & -5 & 0 & 0.0000 & \\
\hline 5 & 5.877869697 & -8.0902 & -4.95 & \multicolumn{2}{|c|}{0.156241286} & -0.9865 \\
\hline 7.1 & 5.746801349 & -7.9098 & -4.75 & \multicolumn{2}{|c|}{0.347610517} & -2.1947 \\
\hline 8.8 & 5.436950039 & -7.4833 & -4.35 & \multirow{2}{*}{\multicolumn{2}{|c|}{$\begin{array}{l}0.554800082 \\
0.784956619\end{array}$}} & -3.5028 \\
\hline 10.3 & 4.98441964 & -6.8604 & -3.65 & & & -4.9559 \\
\hline 11.6 & 4.415841298 & -6.0779 & -2.8 & \multicolumn{2}{|c|}{0.978950416} & -6.1807 \\
\hline 12.8 & 3.678250442 & -5.0627 & -1.6 & \multicolumn{2}{|c|}{1.175257026} & -7.4202 \\
\hline 13.65 & 2.949349614 & -4.0594 & -0.3 & \multicolumn{2}{|c|}{$\begin{array}{l}1.326581688 \\
1447020693\end{array}$} & -8.3756 \\
\hline 14.35 & 2.084573043 & -2.8692 & 1.2 & & 20693 & -9.1360 \\
\hline 14.75 & 1.306091216 & -1.7977 & 2.9 & \multicolumn{2}{|c|}{1.529486276} & -9.6566 \\
\hline 14.95 & 0.587051776 & -0.8080 & 5 & \multicolumn{2}{|c|}{1.564369549} & -9.8769 \\
\hline 15 & $\begin{array}{ll}0 & 0.0000\end{array}$ & & 7.1 & \multicolumn{2}{|c|}{ I.529486276 } & -9.6566 \\
\hline-5 & $\begin{array}{ll}0 & 0.0000\end{array}$ & & 8.8 & \multicolumn{2}{|c|}{1.447020693} & -9.1360 \\
\hline-4.95 & 0.453424664 & -0.8899 & 10.3 & \multicolumn{2}{|c|}{1.326581688} & -8.3756 \\
\hline-4.75 & 1.008793422 & -1.9799 & 11.6 & \multicolumn{2}{|c|}{1.175257026} & -7.4202 \\
\hline-4.35 & 1.61007405 & -3.1599 & 12.8 & \multicolumn{2}{|c|}{0.978950416} & -6.1807 \\
\hline-3.65 & 2.278006661 & -4.4708 & 13.65 & \multicolumn{2}{|c|}{0.784956619} & -4.9559 \\
\hline-2.8 & 2.840992119 & -5.5757 & 14.35 & \multicolumn{2}{|c|}{0.554800082} & -3.5028 \\
\hline-1.6 & 3.410689545 & -6.6938 & 14.75 & & 10517 & -2.1947 \\
\hline-0.3 & 3.849845773 & -7.5557 & 14.95 & & 41286 & -0.9865 \\
\hline 1.2 & 4.199369362 & -8.2417 & 15 & 0 & 0.0000 & \\
\hline 2.9 & 4.438691057 & -8.7114 & -5 & 0 & 0.0000 & \\
\hline 5 & 4.539925094 & -8.9101 & -4.95 & 0 & -0.9987 & \\
\hline 7.1 & 4.438691057 & -8.7114 & -4.75 & 0 & -2.2220 & \\
\hline 8.8 & 4.199369362 & -8.2417 & -4.35 & 0 & -3.5465 & \\
\hline 10.3 & 3.849845773 & -7.5557 & -3.65 & 0 & -5.0177 & \\
\hline 11.6 & 3.410689545 & -6.6938 & -2.8 & 0 & -6.2578 & \\
\hline 12.8 & 2.840992119 & -5.5757 & -1.6 & 0 & -7.5127 & \\
\hline 13.65 & 2.278006661 & -4.4708 & -0.3 & 0 & -8.4800 & \\
\hline 14.35 & 1.61007405 & -3.1599 & 1.2 & 0 & -9.2499 & \\
\hline 14.75 & 1.008793422 & -1.9799 & 2.9 & 0 & -9.7770 & \\
\hline 14.95 & 0.453424664 & -0.8899 & 5 & 0 & -10.0000 & \\
\hline 15 & $\begin{array}{ll}0 & 0.0000\end{array}$ & & 7.1 & 0 & -9.7770 & \\
\hline-5 & $\begin{array}{ll}0 & 0.0000\end{array}$ & & 8.8 & 0 & -9.2499 & \\
\hline-4.95 & 0.30863275 & -0.9499 & 10.3 & 0 & -8.4800 & \\
\hline-4.75 & 0.686655828 & -2.1133 & 11.6 & 0 & -7.5127 & \\
\hline-4.35 & 1.095929757 & -3.3729 & 12.8 & 0 & -6.2578 & \\
\hline-3.65 & 1.550571719 & -4.7721 & 13.65 & 0 & -5.0177 & \\
\hline-2.8 & 1.933779259 & -5.9515 & 14.35 & 0 & -3.5465 & \\
\hline-1.6 & 2.321555437 & -7.1450 & 14.75 & 0 & -2.2220 & \\
\hline-0.3 & 2.620476085 & -8.0649 & 14.95 & 0 & -0.9987 & \\
\hline 1.2 & 2.858386448 & -8.7971 & 15 & 0 & 0.0000 & \\
\hline 2.9 & 3.021285644 & -9.2985 & 3 & 1 & & \\
\hline 5 & 3.090192657 & -9.5106 & 15 & 16 & & \\
\hline 7.1 & 3.021285644 & -9.2985 & 0 & 0 & 0.0000 & \\
\hline 8.8 & 2.858386448 & -8.7971 & 1 & 0 & 0.2680 & \\
\hline 10.3 & 2.620476085 & -8.0649 & 2.8 & 0 & 0.7503 & \\
\hline 11.6 & 2.321555437 & -7.1450 & 4.5 & 0 & 1.2058 & \\
\hline 12.8 & 1.933779259 & -5.9515 & 5.5 & 0 & 1.4737 & \\
\hline
\end{tabular}




\begin{tabular}{lrrr}
5.95 & 0 & 1.5943 & \\
6.46 & 0 & 1.6700 & \\
6.95 & 0 & 1.6900 & \\
7.45 & 0 & 1.6000 & \\
7.85 & 0 & 1.4675 & \\
8.2 & 0 & 1.3100 & \\
8.5 & 0 & 1.1750 & \\
8.9 & 0 & 0.9950 & \\
9.8 & 0 & 0.5900 & \\
11.11111 & 0 & 0.0000 \\
0 & 0 & 0.0000 & \\
1 & 0.055709891 & 0.2621 \\
2.8 & 0.155987695 & 0.7339 \\
4.5 & 0.25069451 & 1.1794 \\
5.5 & 0.306404402 & 1.4415 \\
5.95 & 0.331473853 & 1.5595 \\
6.46 & 0.347212235 & 1.6335 \\
6.95 & 0.351370465 & 1.6531 \\
7.45 & 0.332658428 & 1.5650 \\
7.85 & 0.305110152 & 1.4354 \\
8.2 & 0.272364088 & 1.2814 \\
8.5 & 0.244296033 & 1.1493 \\
8.9 & 0.20687196 & 0.9733 \\
9.8 & 0.122667795 & 0.5771 \\
11.11111 & 0 & 0.0000 \\
0 & 0 & 0.0000 & \\
1 & 0.108984997 & 0.2448 \\
2.8 & 0.305157991 & 0.6854 \\
4.5 & 0.490432486 & 1.1015 \\
5.5 & 0.599417483 & 1.3463 \\
5.95 & 0.648460732 & 1.4565 \\
6.46 & 0.679249654 & 1.5256 \\
6.95 & 0.687384381 & 1.5439 \\
7.45 & 0.650778112 & 1.4617 \\
7.85 & 0.596885549 & 1.3406 \\
8.2 & 0.532824579 & 1.1967 \\
8.5 & 0.477915176 & 1.0734 \\
8.9 & 0.404702638 & 0.9090 \\
9.8 & 0.239974429 & 0.5390 \\
11.11111 & 0 & 0.0000 \\
0 & 0 & 0.0000 & \\
1 & 0.157496943 & 0.2168 \\
2.8 & 0.440991441 & 0.6070 \\
4.5 & 0.708736245 & 0.9755 \\
5.5 & 0.866233188 & 1.1923 \\
5.95 & 0.937106813 & 1.2898 \\
6.46 & 0.981600654 & 1.3511 \\
6.95 & 0.993356351 & 1.3672 \\
7.45 & 0.940455717 & 1.2944 \\
7.85 & 0.862574228 & 1.1872 \\
8.2 & 0.769998118 & 1.0598 \\
8.5 & 0.690647167 & 0.9506 \\
8.9 & 0.584845899 & 0.8050 \\
9.8 & 0.346793046 & 0.4773 \\
\hline & &
\end{tabular}

\begin{tabular}{|c|c|c|}
\hline 11.11111 & \multirow{2}{*}{$\begin{array}{ll}1 & 0 \\
0 & 0.0000\end{array}$} & \multirow[t]{2}{*}{0.0000} \\
\hline $\begin{array}{ll}0 & 0\end{array}$ & & \\
\hline 1 & 0.199125529 & 0.1793 \\
\hline 2.8 & 0.557551482 & 0.5020 \\
\hline 4.5 & 0.896064881 & 0.8068 \\
\hline 5.5 & 1.09519041 & 0.9861 \\
\hline 5.95 & 1.184796898 & 1.0668 \\
\hline 6.46 & 1.241051068 & 1.1174 \\
\hline 6.95 & 1.255913955 & 1.1308 \\
\hline 7.45 & 1.189030963 & 1.0706 \\
\hline 7.85 & 1.090564337 & 0.9819 \\
\hline 8.2 & 0.973519101 & 0.8766 \\
\hline 8.5 & 0.873194614 & 0.7862 \\
\hline 8.9 & 0.73942863 & 0.6658 \\
\hline $9.8 \quad 0$ & 0.438455168 & 0.3948 \\
\hline 11.11111 & $1 \quad 0$ & 0.0000 \\
\hline $\begin{array}{ll}0 & 0\end{array}$ & \multicolumn{2}{|c|}{$\begin{array}{ll}0 & 0.0000\end{array}$} \\
\hline 1 & 0.232051388 & 0.1340 \\
\hline 2.8 & 0.649743888 & 0.3751 \\
\hline 4.5 & 1.044231248 & 0.6029 \\
\hline 5.5 & 1.276282636 & 0.7369 \\
\hline 5.95 & 1.380705761 & 0.7972 \\
\hline 6.46 & 1.446261686 & 0.8350 \\
\hline 6.95 & 1.463582185 & 0.8450 \\
\hline 7.45 & 1.385639938 & 0.8000 \\
\hline 7.85 & 1.270891631 & 0.7338 \\
\hline 8.2 & 1.1344927 & 0.6550 \\
\hline 8.5 & 1.01757933 & 0.5875 \\
\hline 8.9 & 0.861694837 & 0.4975 \\
\hline $9.8 \quad 0$ & 0.510954727 & 0.2950 \\
\hline 11.11111 & $1 \quad 0$ & 0.0000 \\
\hline $0 \quad 0$ & $0 \quad 0.0000$ & \\
\hline 1 & 0.254835506 & 0.0828 \\
\hline 2.8 & 0.713539416 & 0.2318 \\
\hline 4.5 & 1.146759775 & 0.3726 \\
\hline 5.5 & 1.401595281 & 0.4554 \\
\hline 5.95 & 1.516271259 & 0.4927 \\
\hline 6.46 & 1.588263834 & 0.5161 \\
\hline 6.95 & 1.607284958 & 0.5222 \\
\hline 7.45 & 1.521689901 & 0.4944 \\
\hline 7.85 & 1.395674956 & 0.4535 \\
\hline 8.2 & 1.245883607 & 0.4048 \\
\hline 8.5 & 1.117491021 & 0.3631 \\
\hline 8.9 & 0.946300907 & 0.3075 \\
\hline $9.8 \quad 0$ & 0.561123151 & 0.1823 \\
\hline 11.11111 & 0 & 0.0000 \\
\hline $\begin{array}{ll}0 & 0\end{array}$ & $\begin{array}{ll}0 & 0.0000\end{array}$ & \\
\hline 1 & 0.266482107 & 0.0280 \\
\hline 2.8 & 0.7461499 & 0.0784 \\
\hline 4.5 & 1.199169482 & 0.1260 \\
\hline 5.5 & 1.465651589 & 0.1540 \\
\hline 5.95 & 1.585568538 & 0.1667 \\
\hline 6.46 & 1.660851349 & 0.1746 \\
\hline 6.95 & 1.680741784 & 0.1767 \\
\hline
\end{tabular}




\begin{tabular}{|c|c|c|}
\hline 7.45 & 1.591234825 & 0.1672 \\
\hline 7.85 & 1.459460691 & 0.1534 \\
\hline 8.2 & 1.302823513 & 1369 \\
\hline & 1.168563075 & 1228 \\
\hline & 0.989549157 & 0.1040 \\
\hline 8 & 0.586767842 & 0.0617 \\
\hline 11.11111 & $1 \quad 0$ & 0.0000 \\
\hline 0 & 0.0000 & \\
\hline & 0.266482182 & -0.0280 \\
\hline 2.8 & 0.746150108 & -0.0784 \\
\hline 4.5 & 1.199169817 & -0.1260 \\
\hline 5.5 & 1.465651998 & -0.1540 \\
\hline 5.95 & 6898 & -0.1666 \\
\hline 6.46 & 1.66 & \\
\hline 6.95 & 742253 & \\
\hline 7.45 & 1.59 & \\
\hline 7.85 & 1.459 & \\
\hline 8.2 & 1.30 & \\
\hline 8.5 & 1.16 & \\
\hline 8.9 & 0.98 & \\
\hline 9.8 & 68006 & -0.0 \\
\hline 11.11111 & & 0.0000 \\
\hline 0 & 0.0000 & \\
\hline 1 & 0.254835725 & -0.0828 \\
\hline 2.8 & 0.7 & \\
\hline 4.5 & 1.14 & \\
\hline 5.5 & 1.40 & \\
\hline 5.95 & 2566 & -0.4927 \\
\hline 6.46 & 1.58 & \\
\hline 6.95 & 1.60 & \\
\hline 7.45 & 1.52 & \\
\hline 7.85 & 1.39 & \\
\hline 8.2 & 4681 & \\
\hline 8.5 & 1985 & \\
\hline 8.9 & 0.94 & -0.30 \\
\hline 9.8 & 23635 & -0.1823 \\
\hline 11.11111 & & 0.0000 \\
\hline 0 & 0.0000 & \\
\hline 1 & 051744 & -0.1340 \\
\hline 2.8 & 0.64 & 51 \\
\hline 4.5 & 1.0442 & \\
\hline 5.5 & 1.276284592 & -0.7369 \\
\hline 5.95 & 1.380707877 & -0.7971 \\
\hline 6.46 & & \\
\hline & & -0.8450 \\
\hline 7.45 & 12061 & -0.8000 \\
\hline 7.85 & 1.270893578 & -0.7337 \\
\hline 8.2 & 1.134494438 & \\
\hline 8.5 & 1.017580889 & -0.5875 \\
\hline 8.9 & 0.861696157 & -0.4975 \\
\hline 9.8 & 0.51095551 & -0.2950 \\
\hline 11.11111 & $1 \quad 0$ & 0.0000 \\
\hline $0 \quad c$ & 0.0000 & \\
\hline 1 & 0.199126005 & -0.1793 \\
\hline
\end{tabular}

$\begin{array}{lcc}2.8 & 0.557552814 & -0.5020 \\ 4.5 & 0.896067022 & -0.8068 \\ 5.5 & 1.095193027 & -0.9861 \\ 5.95 & 1.184799729 & -1.0668 \\ 6.46 & 1.241054033 & -1.1174 \\ 6.95 & 1.255916956 & -1.1308 \\ 7.45 & 1.189033804 & -1.0706 \\ 7.85 & 1.090566942 & -0.9819 \\ 8.2 & 0.973521427 & -0.8766 \\ 8.5 & 0.8731967 & -0.7862 \\ 8.9 & 0.739430397 & -0.6658 \\ 9.8 & 0.438456215 & -0.3948 \\ 11.11111 & 0 & 0.0000\end{array}$

$\begin{array}{lll}0 & 0 & 0.0000\end{array}$

$\begin{array}{llll}1 & 0.157497519 & -0.2168\end{array}$

$\begin{array}{lll}2.8 & 0.440993052 & -0.6070\end{array}$

$\begin{array}{lll}4.5 & 0.708738833 & -0.9755\end{array}$

$\begin{array}{lll}5.5 & 0.866236352 & -1.1923\end{array}$

$\begin{array}{lll}5.95 & 0.937110235 & -1.2898\end{array}$

$\begin{array}{lll}6.46 & 0.981604239 & -1.3511\end{array}$

$\begin{array}{lll}6.95 & 0.993359979 & -1.3672\end{array}$

$\begin{array}{lll}7.45 & 0.940459152 & -1.2944\end{array}$

$\begin{array}{lll}7.85 & 0.862577378 & -1.1872\end{array}$

$\begin{array}{lll}8.2 & 0.77000093 & -1.0598\end{array}$

$\begin{array}{lll}8.5 & 0.690649689 & -0.9506\end{array}$

$8.9 \quad 0.584848035 \quad-0.8050$

$\begin{array}{lll}9.8 & 0.346794312 & -0.4773\end{array}$

$\begin{array}{lll}11.11111 & 0 & 0.0000\end{array}$

$\begin{array}{llll}0 & 0 & 0.0000 & \\ 1 & 0.108985646 & -0.2448\end{array}$

$\begin{array}{lll}2.8 & 0.30515981 & -0.6854\end{array}$

$\begin{array}{lll}4.5 & 0.490435409 & -1.1015\end{array}$

$\begin{array}{lll}5.5 & 0.599421056 & -1.3463\end{array}$

$\begin{array}{lll}5.95 & 0.648464596 & -1.4565\end{array}$

$\begin{array}{lll}6.46 & 0.679253703 & -1.5256\end{array}$

$\begin{array}{lll}6.95 & 0.687388477 & -1.5439\end{array}$

$\begin{array}{lll}7.45 & 0.65078199 & -1.4617\end{array}$

$\begin{array}{lll}7.85 & 0.596889107 & -1.3406\end{array}$

$\begin{array}{lll}8.2 & 0.532827755 & -1.1967\end{array}$

$\begin{array}{lll}8.5 & 0.477918024 & -1.0734\end{array}$

$\begin{array}{lll}8.9 & 0.40470505 & -0.9090\end{array}$

$\begin{array}{lll}9.8 & 0.239975859 & -0.5390\end{array}$

$\begin{array}{lll}11.11111 & 0 & 0.0000\end{array}$

$\begin{array}{llll}0 & 0 & 0.0000 & \\ 1 & 0.055710587 & -0.2621\end{array}$

$\begin{array}{lll}2.8 & 0.155989643 & -0.7339\end{array}$

$\begin{array}{lll}4.5 & 0.25069764 & -1.1794\end{array}$

$\begin{array}{lll}5.5 & 0.306408227 & -1.4415\end{array}$

$\begin{array}{lll}5.95 & 0.331477991 & -1.5595\end{array}$

$\begin{array}{lll}6.46 & 0.347216569 & -1.6335\end{array}$

$\begin{array}{lll}6.95 & 0.351374852 & -1.6531\end{array}$

$\begin{array}{lll}7.45 & 0.332662581 & -1.5650\end{array}$

$\begin{array}{lll}7.85 & 0.305113961 & -1.4354\end{array}$

$\begin{array}{lll}8.2 & 0.272367489 & -1.2814\end{array}$ 


$\begin{array}{llll}8.5 & 0.244299083 & -1.1493 \\ 8.9 & 0.206874543 & -0.9733 \\ 9.8 & 0.122669327 & -0.5771 \\ 11.11111 & 0 & 0.0000 \\ 0 & 0 & 0.0000 & \\ 1 & 0 & -0.2680 & \\ 2.8 & 0 & -0.7503 & \\ 4.5 & 0 & -1.2058 & \\ 5.5 & 0 & -1.4737 & \\ 5.95 & 0 & -1.5943 & \\ 6.46 & 0 & -1.6700 & \\ 6.95 & 0 & -1.6900 & \\ 7.45 & 0 & -1.6000 & \\ 7.85 & 0 & -1.4675 & \\ 8.2 & 0 & -1.3100 & \\ 8.5 & 0 & -1.1750 & \\ 8.9 & 0 & -0.9950 & \\ 9.8 & 0 & -0.5900 & \\ 11.11111 & 0 & 0.0000 \\ 4 & 1 & & \\ 19 & 2 & & \\ 1.7159 & 0.45985 & 0.0000 & \\ 1.746025415 & 0.4679 & 0.0042 \\ 1.835486318 & 0.4916 & 0.0165 \\ 1.981564487 & 0.5299 & 0.0356 \\ 2.17982142 & 0.5811 & 0.0598 \\ 2.42423319 & 0.6439 & 0.0863 \\ 2.707373481 & 0.7168 & 0.1123 \\ 3.020639234 & 0.7982 & 0.1348 \\ 3.354512043 & 0.8862 & 0.1510 \\ 3.698847369 & 0.9784 & 0.1590 \\ 4.043182775 & 1.072 & 0.1576 \\ 4.377055822 & 1.1638 & 0.1466 \\ 4.690321962 & 1.2505 & 0.1273 \\ 4.973462779 & 1.329 & 0.1016 \\ 5.217875198 & 1.3965 & 0.0729 \\ 5.416132882 & 1.4508 & 0.0448 \\ 5.562211883 & 1.4905 & 0.0212 \\ 5.651673671 & 1.5146 & 0.0055 \\ 5.6818 & 1.5227 & 0.0000 & \\ 3.8975 & 2.4242 & 0.0000 & \\ 3.913010498 & 2.4242 & 0.0031 \\ 3.959070716 & 2.4242 & 0.0120 \\ 4.034281138 & 2.4242 & 0.0255 \\ 4.136356539 & 2.4242 & 0.0422 \\ 4.262195413 & 2.4242 & 0.0599 \\ 4.407974218 & 2.4242 & 0.0766 \\ 4.569263545 & 2.4242 & 0.0902 \\ 4.741162707 & 2.4242 & 0.0990 \\ 4.918448645 & 2.4242 & 0.1021 \\ 5.095734625 & 2.4242 & 0.0990 \\ 5.26763391 & 2.4242 & 0.0902 \\ 5.428923436 & 2.4242 & 0.0766 \\ 5.574702511 & 2.4242 & 0.0599\end{array}$

$\begin{array}{llll}\begin{array}{l}5.70054172 \\ 5.802617507\end{array} & 2.4242 & 0.0422 \\ 5.877828358 & 2.4242 & 0.0255 \\ 5.923889031 & 2.4242 & 0.0119 \\ 5.9394 & 2.4242 & 0.0031 \\ 5 & 1 & & \\ 2 & 2 & & \\ 6.6024 & 4.8597 & 1.0000 & \\ 7.5017 & 4.8597 & 1.0000 & \\ 6.6024 & 4.8597 & -1.0000 & \\ 7.5017 & 4.8597 & -1.0000 & \\ 6 & 1 & & \\ 19 & 2 & & \\ 6.6024 & 4.8597 & 0.0000 & \\ 6.609231182 & 4.8597 & -0.0014 \\ 6.629517168 & 4.8597 & -0.0053 \\ 6.662641578 & 4.8597 & -0.0113 \\ 6.707597946 & 4.8597 & -0.0186 \\ 6.763020297 & 4.8597 & -0.0264 \\ 6.827224656 & 4.8597 & -0.0337 \\ 6.898260207 & 4.8597 & -0.0397 \\ 6.973968575 & 4.8597 & -0.0436 \\ 7.052049403 & 4.8597 & -0.0450 \\ 7.13013025 & 4.8597 & -0.0436 \\ 7.205838672 & 4.8597 & -0.0397 \\ 7.276874311 & 4.8597 & -0.0337 \\ 7.341078789 & 4.8597 & -0.0264 \\ 7.396501287 & 4.8597 & -0.0186 \\ 7.441457826 & 4.8597 & -0.0113 \\ 7.474582424 & 4.8597 & -0.0053 \\ 7.49486861 & 4.8597 & -0.0014 \\ 7.5017 & 4.8597 & 0.0000 & \\ 3.8975 & 2.4242 & 0.0000 & \\ 3.913010498 & 2.4242 & -0.0031 \\ 3.959070716 & 2.4242 & -0.0120 \\ 4.034281138 & 2.4242 & -0.0255 \\ 4.136356539 & 2.4242 & -0.0422 \\ 4.262195413 & 2.4242 & -0.0599 \\ 4.407974218 & 2.4242 & -0.0766 \\ 4.569263545 & 2.4242 & -0.0902 \\ 4.741162707 & 2.4242 & -0.0990 \\ 4.918448645 & 2.4242 & -0.1021 \\ 5.095734625 & 2.4242 & -0.0990 \\ 5.26763391 & 2.4242 & -0.0902 \\ 5.428923436 & 2.4242 & -0.0766 \\ 5.574702511 & 2.4242 & -0.0599 \\ 5.70054172 & 2.4242 & -0.0422 \\ 5.802617507 & 2.4242 & -0.0255 \\ 5.877828358 & 2.4242 & -0.0119 \\ 5.923889031 & 2.4242 & -0.0031 \\ 5.9394 & 2.4242 & 0.0000 & \\ 7 & 1 & & \\ 19 & 2 & & \\ 3.8975 & 2.4242 & 0.0000 & \\ & & & \end{array}$




\begin{tabular}{|c|c|c|c|c|c|c|}
\hline 3.913010498 & 2.4242 & 0.0031 & \multicolumn{2}{|c|}{5.802617507} & 2.4242 & -0.025 \\
\hline 3.959070716 & 2.4242 & 0.0120 & \multicolumn{2}{|c|}{5.877828358} & 2.4242 & -0.011 \\
\hline 4.034281138 & 2.4242 & 0.0255 & \multicolumn{2}{|c|}{5.923889031} & 2.4242 & -0.003 \\
\hline 4.136356539 & 2.4242 & 0.0422 & 5.9394 & 2.4242 & 0.0000 & \\
\hline 4.262195413 & 2.4242 & 0.0599 & 1.7159 & 0.45985 & 0.0000 & \\
\hline 4.407974218 & 2.4242 & 0.0766 & \multicolumn{2}{|c|}{1.746025415} & 0.4679 & -0.004 \\
\hline 4.569263545 & 2.4242 & 0.0902 & \multicolumn{2}{|c|}{1.835486318} & 0.4916 & -0.016 \\
\hline 4.741162707 & 2.4242 & 0.0990 & \multicolumn{2}{|c|}{1.981564487} & 0.5299 & -0.035 \\
\hline 4.918448645 & 2.4242 & 0.1021 & \multicolumn{2}{|c|}{2.17982142} & 0.5811 & -0.059 \\
\hline 5.095734625 & 2.4242 & 0.0990 & \multicolumn{2}{|c|}{2.42423319} & 0.6439 & -0.086 \\
\hline 5.26763391 & 2.4242 & 0.0902 & \multicolumn{2}{|c|}{2.707373481} & 0.7168 & -0.112 \\
\hline 5.428923436 & 2.4242 & 0.0766 & \multicolumn{2}{|c|}{3.020639234} & 0.7982 & -0.134 \\
\hline 5.574702511 & 2.4242 & 0.0599 & \multicolumn{2}{|c|}{3.354512043} & 0.8862 & -0.151 \\
\hline 5.70054172 & 2.4242 & 0.0422 & \multicolumn{2}{|c|}{3.698847369} & 0.9784 & -0.15 \\
\hline 5.802617507 & 2.4242 & 0.0255 & \multicolumn{2}{|c|}{4.043182775} & 1.072 & -0.157 \\
\hline 5.877828358 & 2.4242 & 0.0119 & \multicolumn{2}{|c|}{4.377055822} & 1.1638 & -0.146 \\
\hline 5.923889031 & 2.4242 & 0.0031 & \multicolumn{2}{|c|}{4.690321962} & 1.2505 & -0.127 \\
\hline $5.9394 \quad 2.4242$ & 0.0000 & & \multicolumn{2}{|c|}{4.973462779} & 1.329 & -0.101 \\
\hline $6.6024 \quad 4.8597$ & 0.0000 & & \multicolumn{2}{|c|}{5.217875198} & 1.3965 & -0.072 \\
\hline 6.609231182 & 4.8597 & 0.0014 & \multicolumn{2}{|c|}{5.416132882} & 1.4508 & -0.044 \\
\hline 6.629517168 & 4.8597 & 0.0053 & \multicolumn{2}{|c|}{5.562211883} & 1.4905 & -0.021 \\
\hline 6.662641578 & 4.8597 & 0.0113 & 5.65167 & 3671 & 1.5146 & -0.005 \\
\hline 6.707597946 & 4.8597 & 0.0186 & 5.6818 & 1.5227 & 0.0000 & \\
\hline 6.763020297 & 4.8597 & 0.0264 & Mesh ge & eneration & & \\
\hline 6.827224656 & 4.8597 & 0.0337 & 14 & 8 & & \\
\hline 6.898260207 & 4.8597 & 0.0397 & segment & & in & curves \\
\hline 6.973968575 & 4.8597 & 0.0436 & 1 & 1 & 1 & \\
\hline 7.052049403 & 4.8597 & 0.0450 & 2 & 2 & 1 & \\
\hline 7.13013025 & 4.8597 & 0.0436 & 3 & 3 & 1 & \\
\hline 7.205838672 & 4.8597 & 0.0397 & 4 & 4 & 1 & \\
\hline 7.276874311 & 4.8597 & 0.0337 & 5 & 5 & 1 & \\
\hline 7.341078789 & 4.8597 & 0.0264 & 6 & 6 & 1 & \\
\hline 7.396501287 & 4.8597 & 0.0186 & 7 & 7 & 1 & \\
\hline 7.441457826 & 4.8597 & 0.0113 & 8 & 8 & 1 & \\
\hline 7.474582424 & 4.8597 & 0.0053 & 9 & 9 & 1 & \\
\hline 7.49486861 & 4.8597 & 0.0014 & 10 & 10 & 1 & \\
\hline $\begin{array}{ll}7.5017 & 4.8597\end{array}$ & 0.0000 & & 11 & 11 & 1 & \\
\hline $\begin{array}{ll}8 & 1\end{array}$ & & & 12 & 12 & 1 & \\
\hline $19 \quad 2$ & & & 13 & 13 & 1 & \\
\hline $\begin{array}{ll}3.8975 & 2.4242\end{array}$ & 0.0000 & & 14 & 14 & 1 & \\
\hline 3.913010498 & 2.4242 & -0.0031 & Regions & on & Surfaces & \\
\hline 3.959070716 & 2.4242 & -0.0120 & 1 & 1 & 1 & \\
\hline 4.034281138 & 2.4242 & -0.0255 & 4 & & & \\
\hline 4.136356539 & 2.4242 & -0.0422 & 4 & 3 & 1 & 2 \\
\hline 4.262195413 & 2.4242 & -0.0599 & 2 & 2 & 1 & \\
\hline 4.407974218 & 2.4242 & -0.0766 & 2 & & & \\
\hline 4.569263545 & 2.4242 & -0.0902 & 1 & 2 & & \\
\hline 4.741162707 & 2.4242 & -0.0990 & 3 & 3 & 1 & \\
\hline 4.918448645 & 2.4242 & -0.1021 & 4 & & & \\
\hline 5.095734625 & 2.4242 & -0.0990 & 3 & 4 & 5 & 6 \\
\hline 5.26763391 & 2.4242 & -0.0902 & 4 & 4 & 1 & \\
\hline 5.428923436 & 2.4242 & -0.0766 & 4 & & & \\
\hline 5.574702511 & 2.4242 & -0.0599 & 5 & 7 & 12 & 14 \\
\hline 5.70054172 & 2.4242 & -0.0422 & 5 & 5 & 1 & \\
\hline
\end{tabular}




$\begin{array}{llll}2 & & & \\ 9 & 10 & & \\ 6 & 6 & 1 & \\ 4 & & & \\ 11 & 10 & 8 & 13 \\ 7 & 7 & 1 & \\ 4 & & & \\ 12 & 8 & 9 & 11 \\ 8 & 8 & 1 & \\ 4 & & & \\ 13 & 7 & 6 & 14\end{array}$




\section{CSWC Boundary Condition File}

$\begin{array}{lc}\text { CSWC Boundary Condition File } \\ 8 & 14 \\ \text { Surface Regions } \\ 1 & 1 \\ 2 & 3 \\ 3 & 1 \\ 4 & 1 \\ 5 & 1 \\ 6 & 1 \\ 7 & 1 \\ 8 & 1 \\ \text { Curve Segments } \\ 1 & 0 \\ 2 & 0 \\ 3 & 0 \\ 4 & 0 \\ 5 & 0 \\ 6 & 0 \\ 7 & 0 \\ 8 & 0 \\ 9 & 0 \\ 10 & 0 \\ 11 & 0 \\ 12 & 0 \\ 13 & 0 \\ 14 & 0\end{array}$




\section{CSWC Background File}

\begin{tabular}{|c|c|c|c|c|c|c|}
\hline \multicolumn{2}{|c|}{ background } & \multicolumn{5}{|c|}{ Mesh......X-35 } \\
\hline 4 & 1 & 1 & 4 & 0 & & \\
\hline \multirow[t]{4}{*}{1} & -20 & -5 & -20 & & & \\
\hline & 1 & 0 & 0 & 1.00 & & \\
\hline & 0 & 1 & 0 & 1.00 & & \\
\hline & 0 & 0 & 1 & 1.00 & & \\
\hline \multirow[t]{4}{*}{2} & 20 & -5 & -20 & & & \\
\hline & 1 & 0 & 0 & 1.00 & & \\
\hline & 0 & 1 & 0 & 1.00 & & \\
\hline & 0 & 0 & 1 & 1.00 & & \\
\hline \multirow[t]{4}{*}{3} & 5 & -5 & 20 & & & \\
\hline & 1 & 0 & 0 & 1.00 & & \\
\hline & 0 & 1 & 0 & 1.00 & & \\
\hline & 0 & 0 & 1 & 1.00 & & \\
\hline \multirow[t]{4}{*}{4} & 5 & 20 & 0 & & & \\
\hline & 1 & 0 & 0 & 1.00 & & \\
\hline & 0 & 1 & 0 & 1.00 & & \\
\hline & 0 & 0 & 1 & 1.00 & & \\
\hline 1 & 1 & 2 & 3 & 4 & & \\
\hline * & Point & Source & & & & \\
\hline \multicolumn{7}{|c|}{ 2.-Shock catcher } \\
\hline 0.3 & 0.08 & 0 & 0.05 & 0.20 & & 1.1 \\
\hline * & Line & Source & & & & \\
\hline \multicolumn{7}{|c|}{ Leading Edge of the wing from point $1->3$} \\
\hline 1.7159 & 0.4598 & 0.00 & 0.08 & 0.24 & & 1.36 \\
\hline 3.8975 & 2.4242 & 0.00 & 0.08 & 0.24 & 1.20 & \\
\hline \multicolumn{7}{|c|}{ Leading Edge of the wing from point $3->5$} \\
\hline 3.8975 & 2.4242 & 0.00 & 0.08 & 0.24 & 1.20 & \\
\hline 6.6024 & 4.8597 & 0.00 & 0.08 & 0.24 & 0.56 & \\
\hline \multicolumn{7}{|c|}{ Trailing Edge of the wing from point $4->6$} \\
\hline 5.9394 & 2.4242 & 0.00 & 0.08 & 0.24 & 0.56 & \\
\hline 7.5017 & 4.8597 & 0.00 & 0.08 & 0.24 & 0.40 & \\
\hline \multicolumn{7}{|c|}{ Trailing Edge of the wing from point 2->4 } \\
\hline 5.6818 & 1.5226 & 0.00 & 0.08 & 0.24 & 0.72 & \\
\hline 5.9394 & 2.4242 & 0.00 & 0.08 & 0.24 & 0.72 & \\
\hline
\end{tabular}




\section{APPENDIX C: MODE 1 TRANSIENT ANALYSIS}

Note, for the following charts, the horizontal axis is time and the vertical is amplitude.

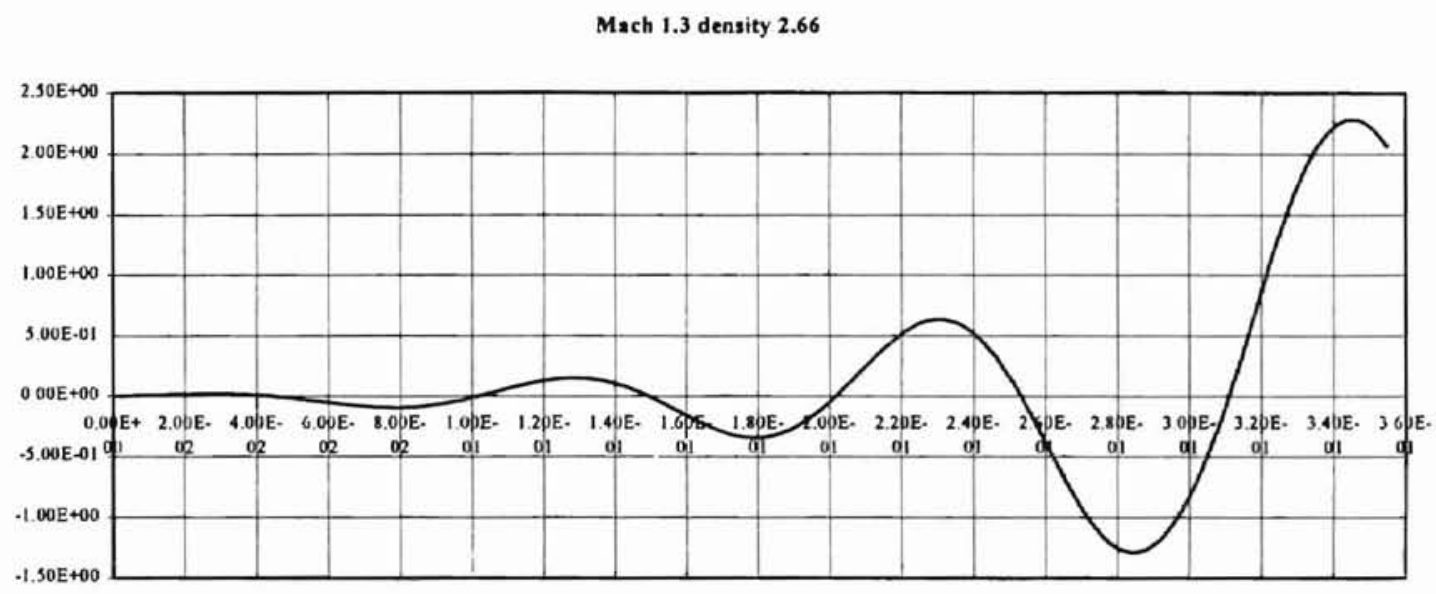

Mach 1.3 Density 2.0

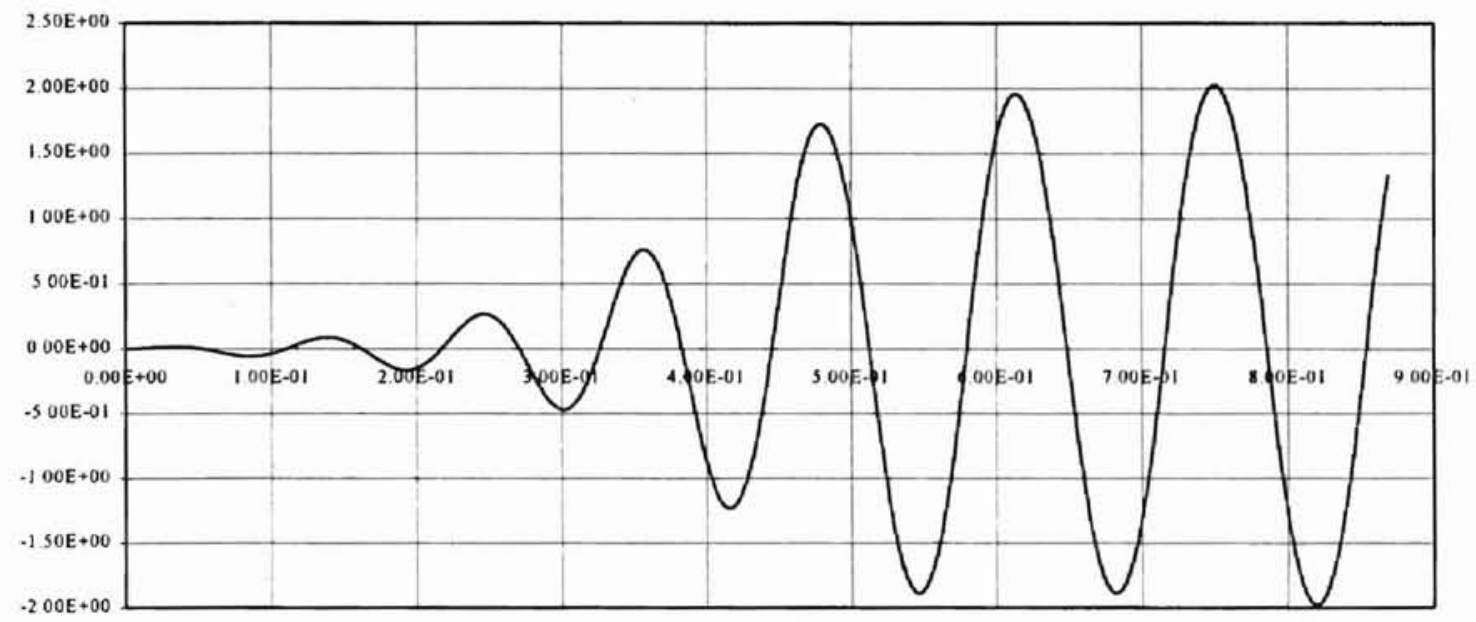


Mach 1.3 Density 1.6
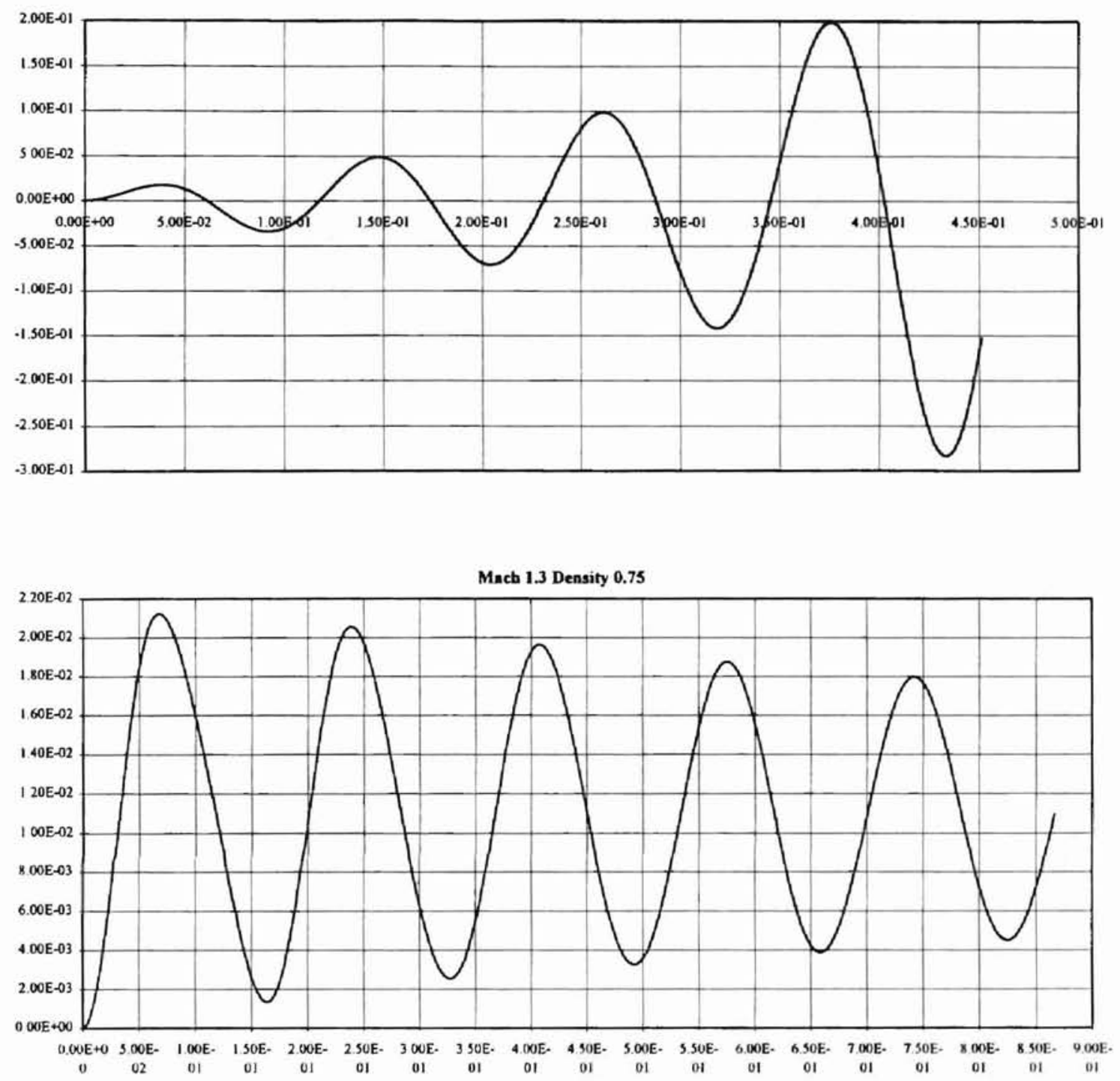
Mach 1.6 Density 0.9

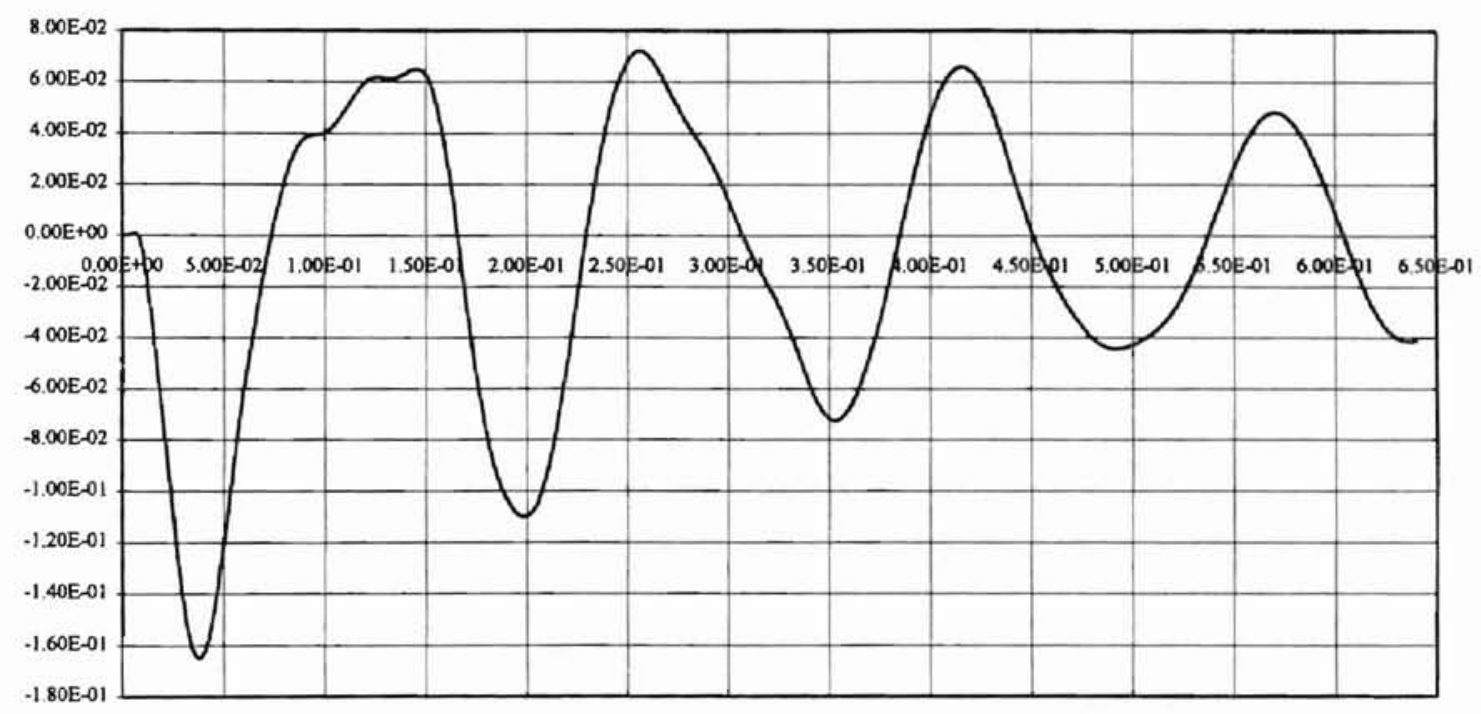

Mach 1.6 Density 1.6

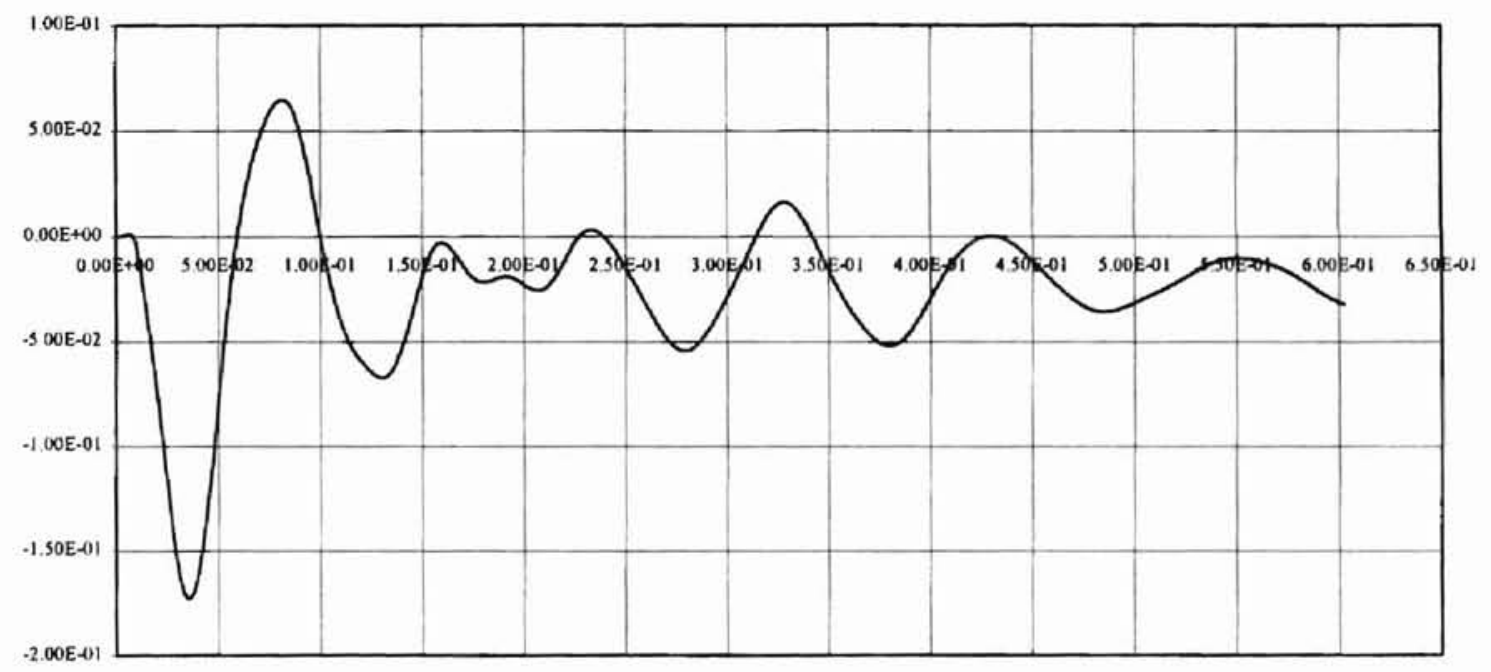

96 
Mach 1.6 Density 1.94

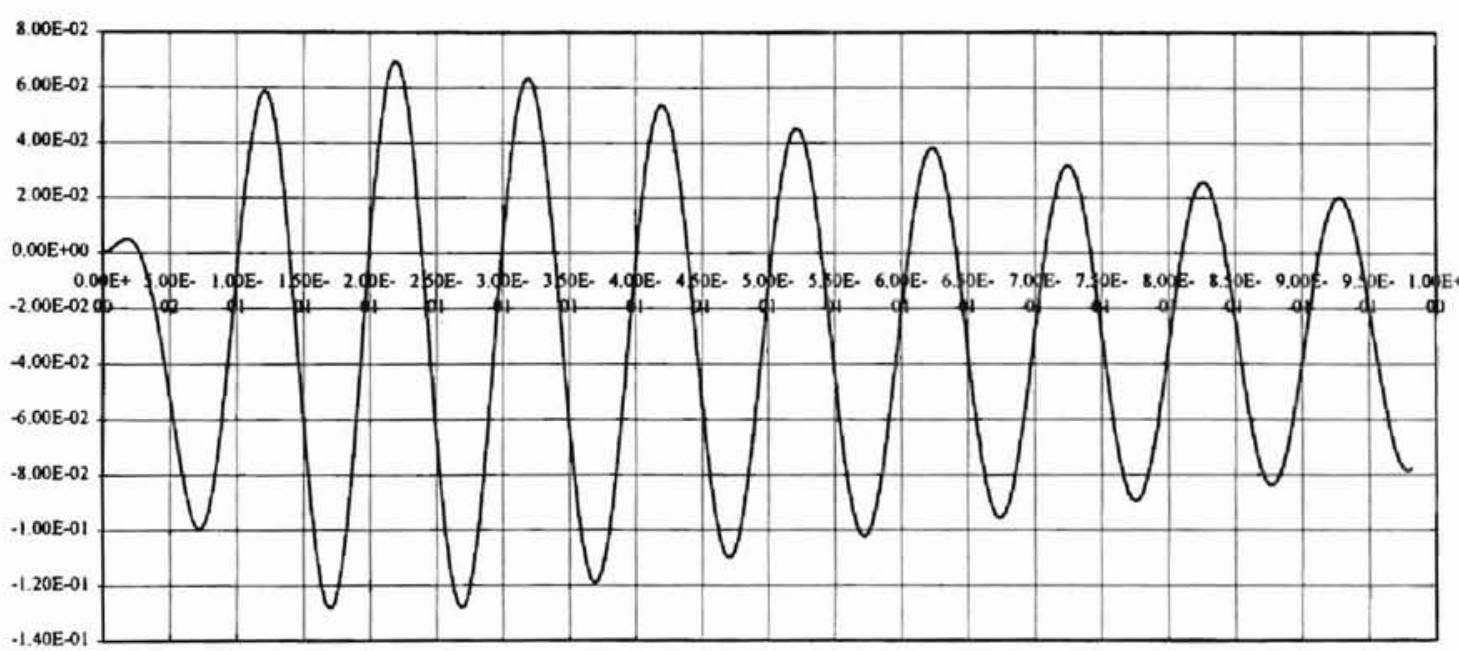

Mach 1.6 Deasity 2.09

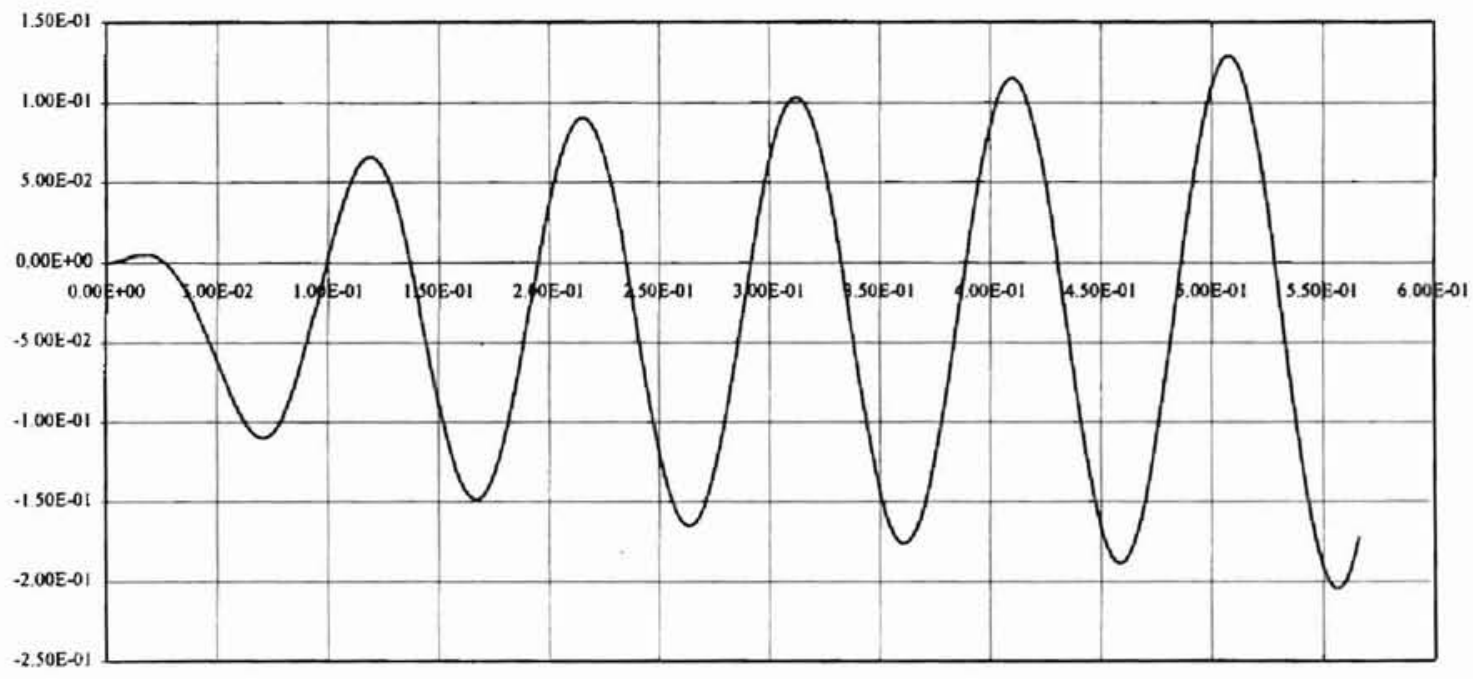


Mach 2.0 Density 1.02

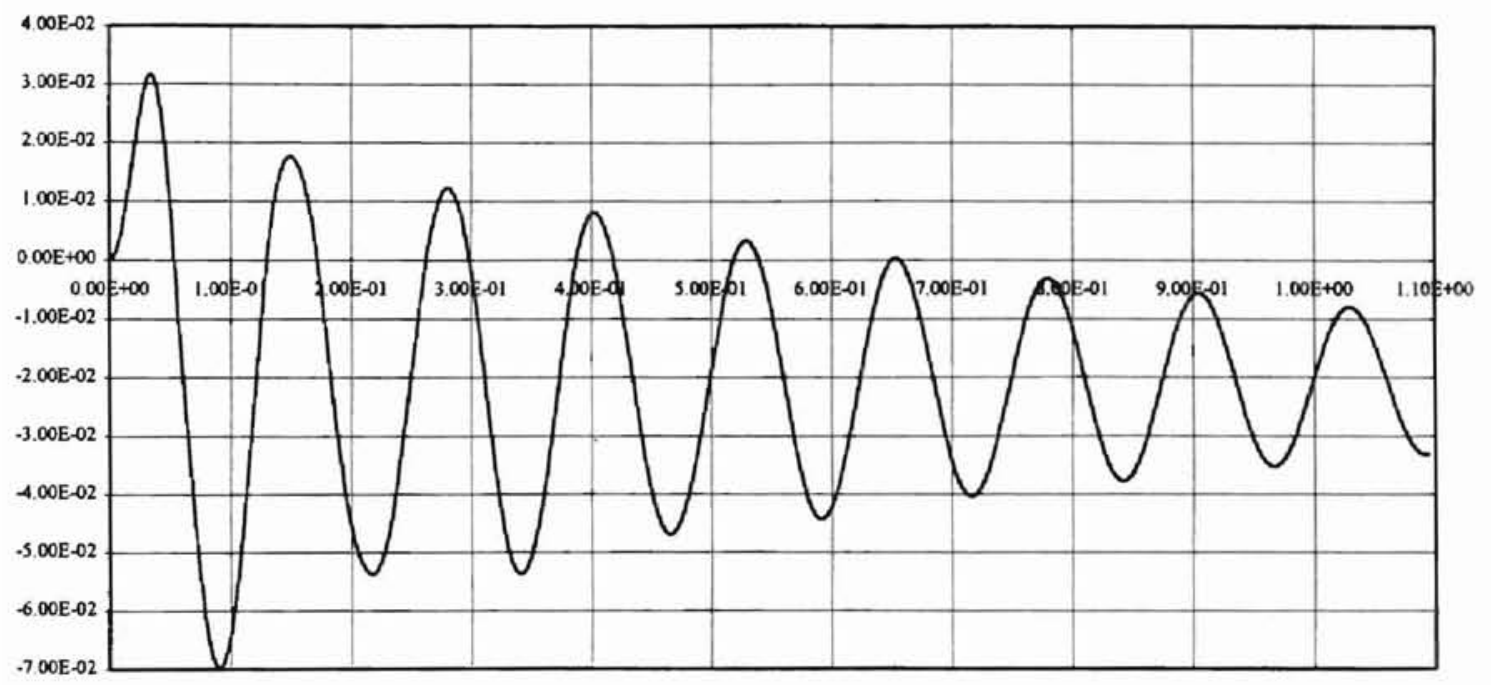

Mach 2.0 Density 1.1

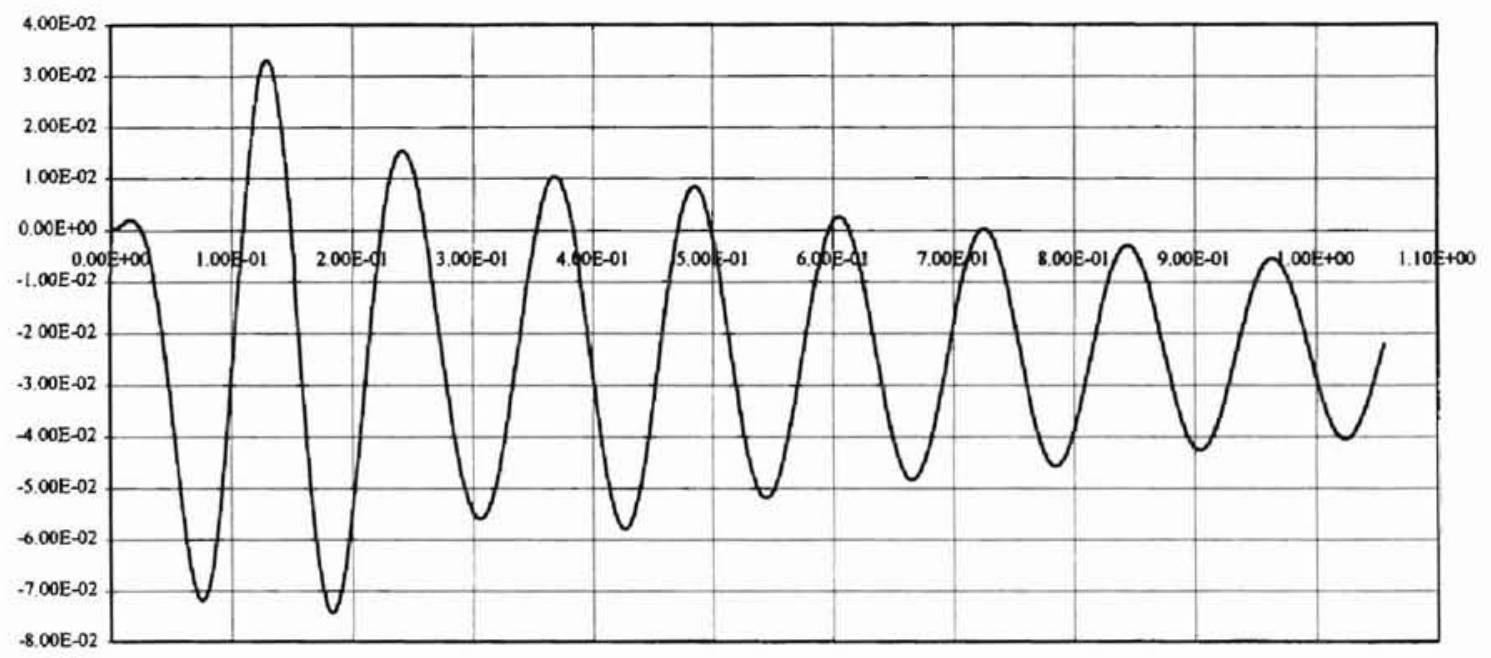

98 
Mach 2.0 Density 1.2

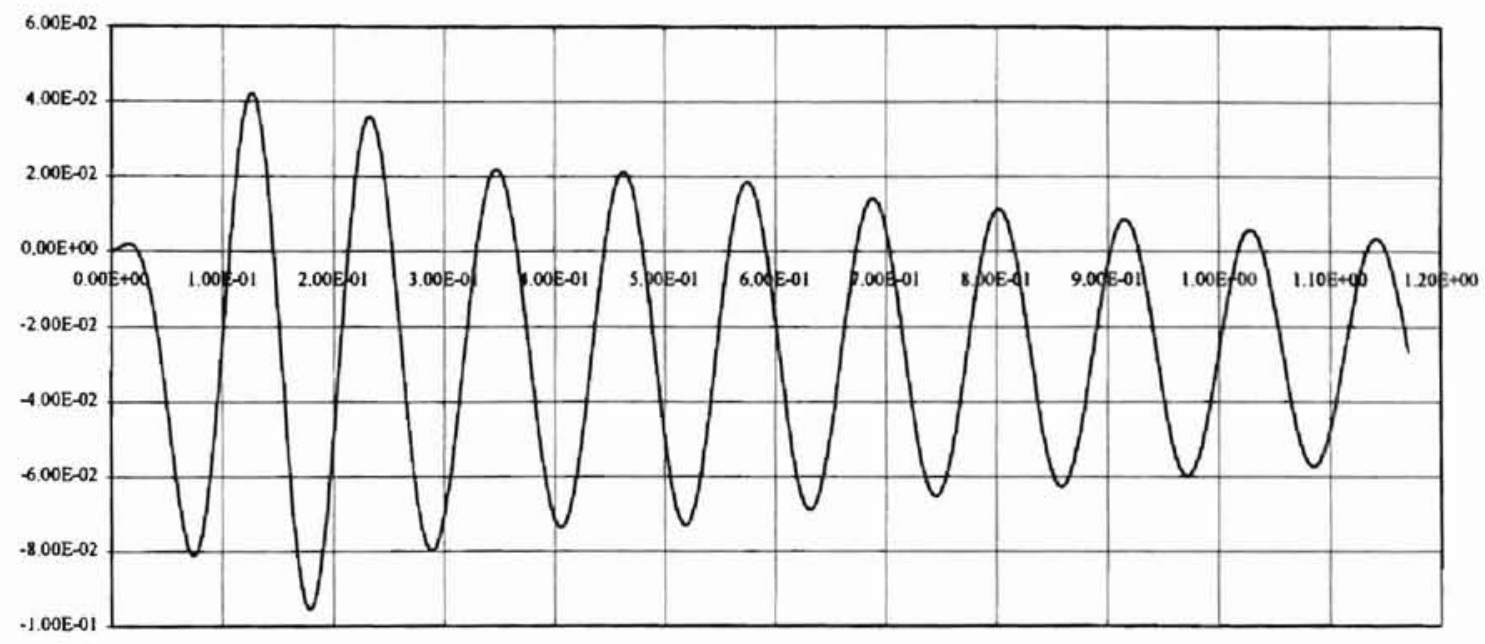

Mach 2.0 Density 1.4

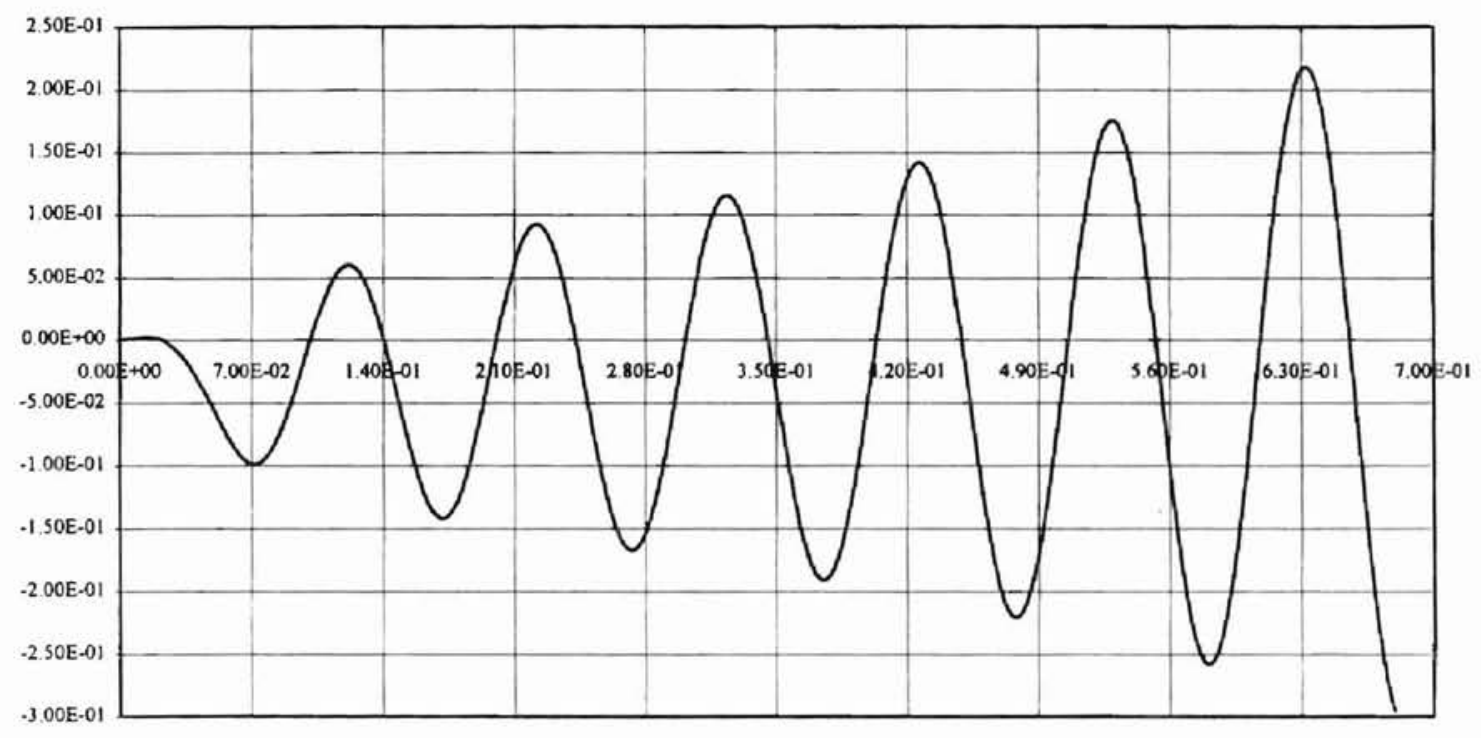

99 
Mach 2.4 Density 0.7

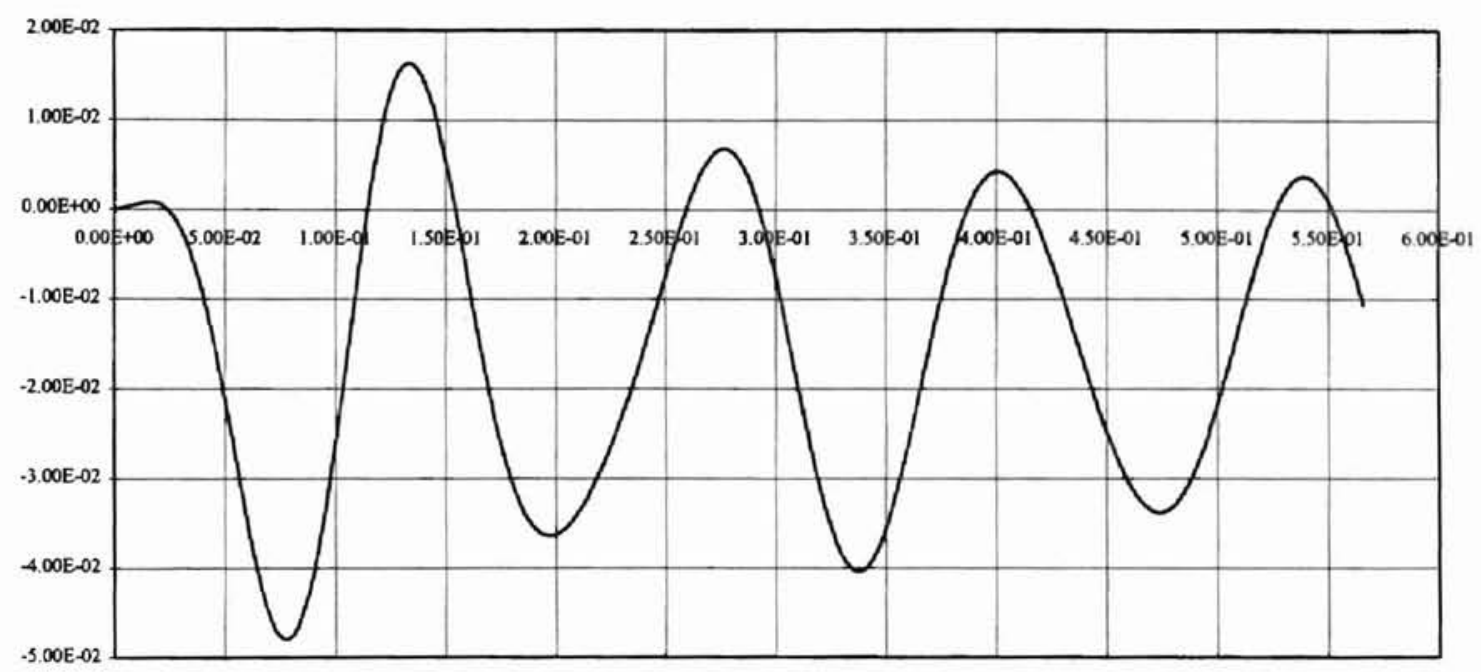

Mach 2.4 Density 0.8

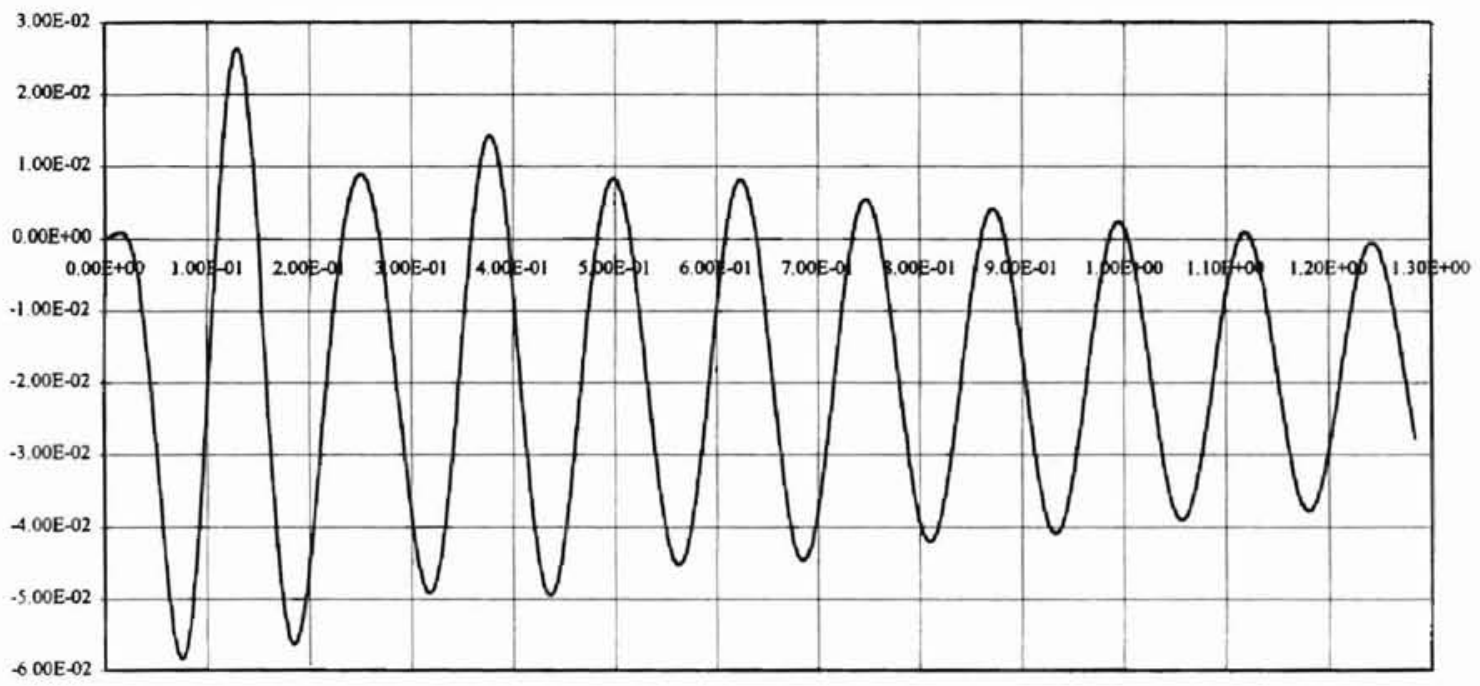

100 
Mach 2.4 density 1.0

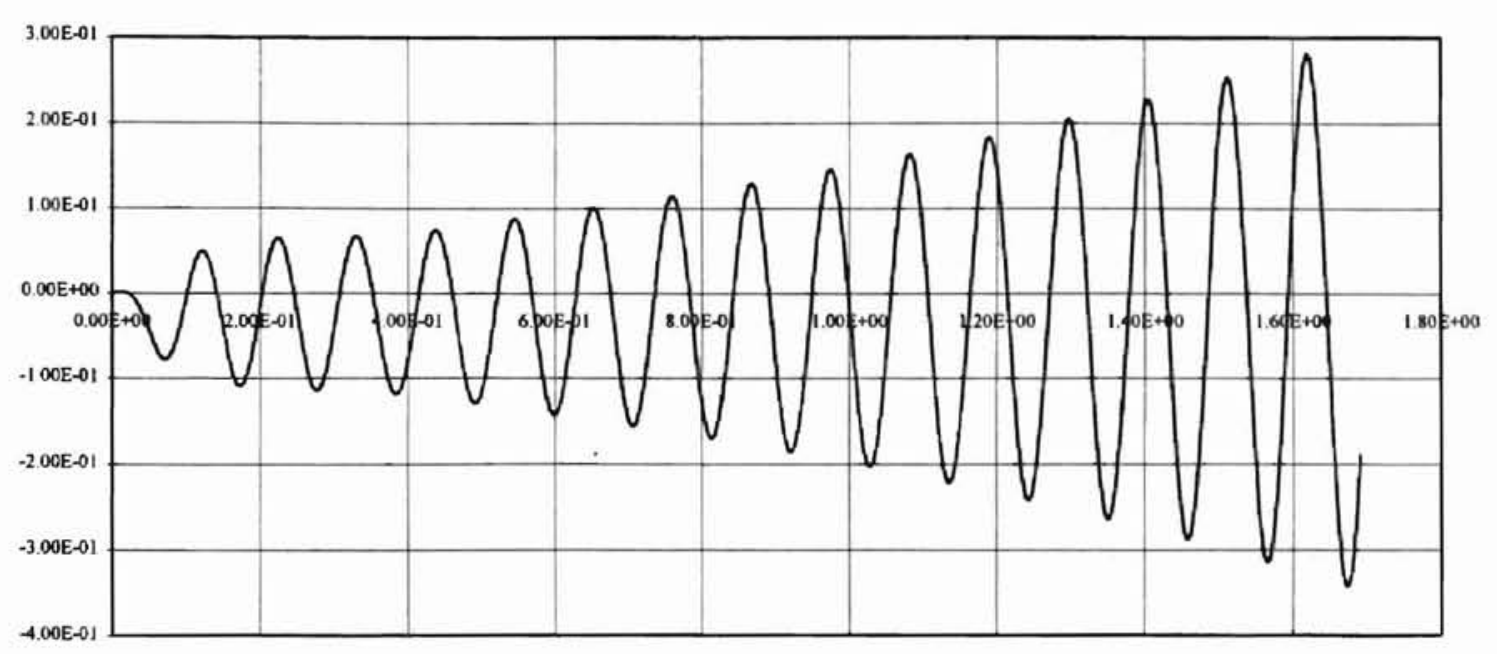

Mach 2.8 Density 0.6

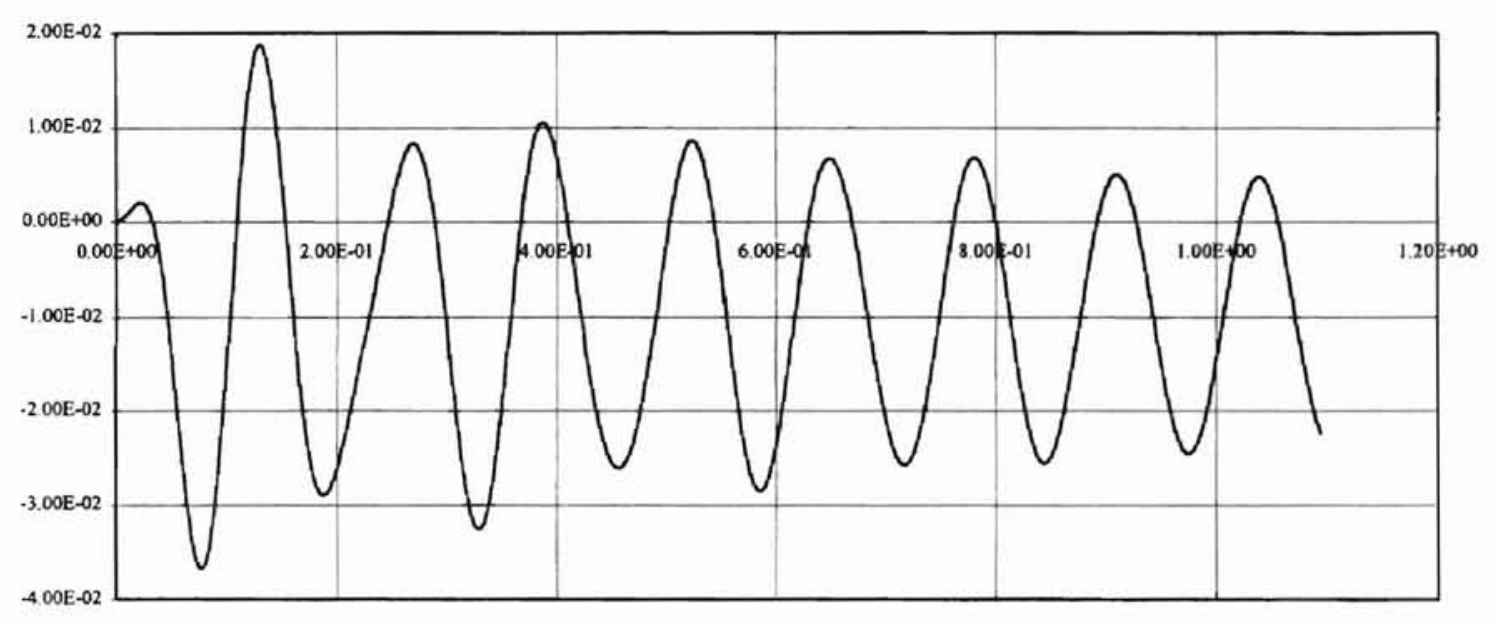


Mach 2.8 Density 0.69

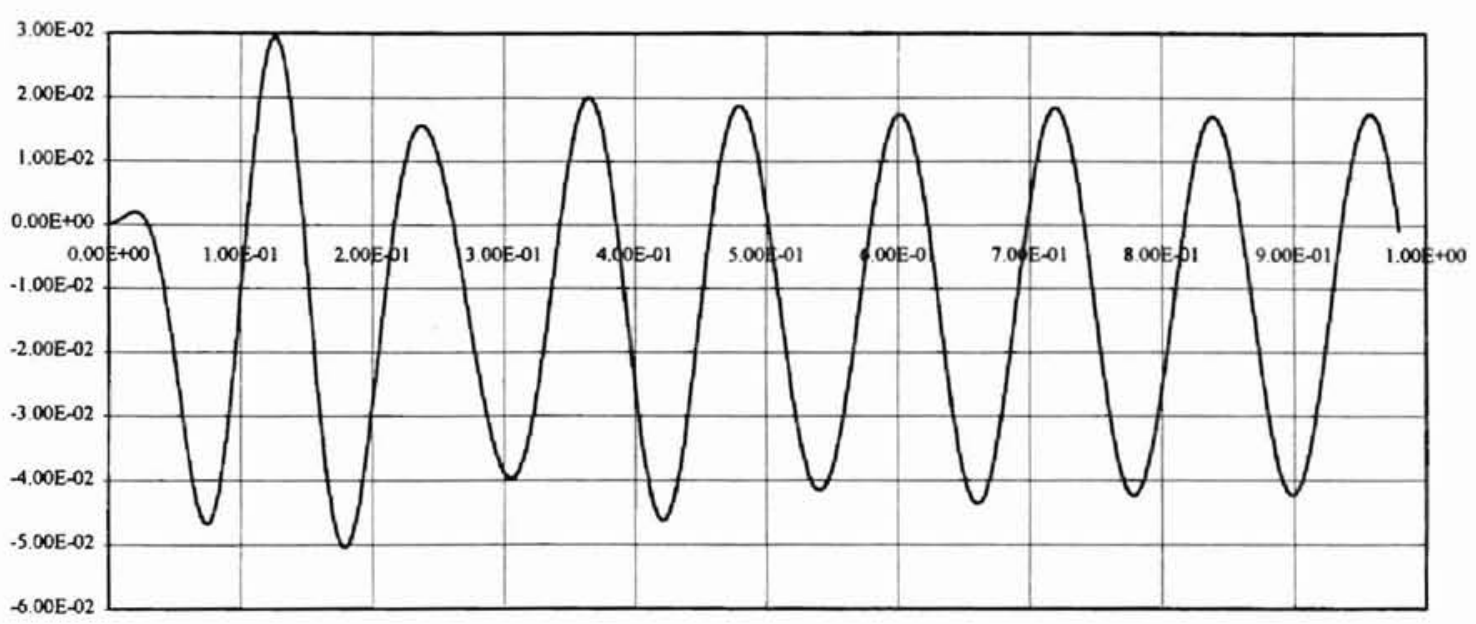

Mach 2.8 Density 0.79

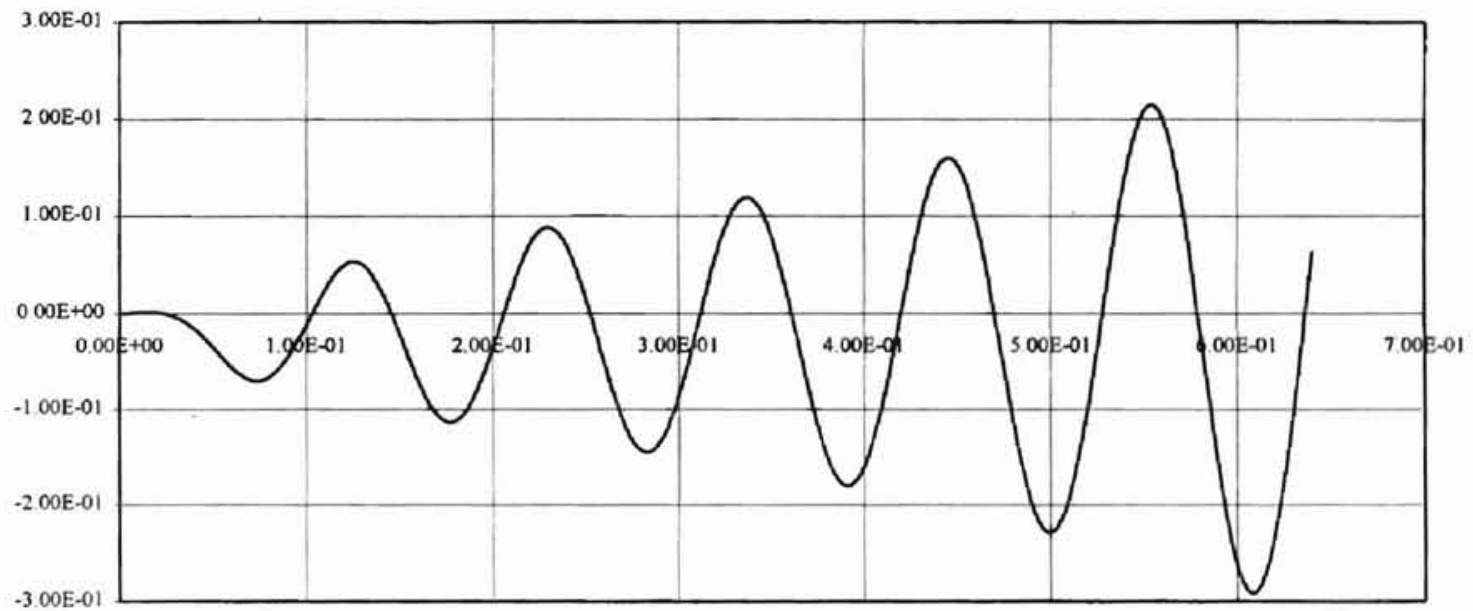




\section{VITA}

John Paul Hunter

Candidate for the Degree of

Master of science

\section{Thesis: AN EFFICIENT METHOD FOR TIME-MARCHING SUPERSONIC FLUTTER PREDICTIONS USING CFD}

Major Field: Mechanical Engineering

Biographical:

Personal data: Born in Queens, New York on April 21, 1969, the son of Charlie Sciara and Theressa Newman. Adopted at the age of 4 by Dr. Mary Paula Hunter.

Education: Graduated from Ponca City High School in Ponca City, Oklahoma on May 1987; entered the Air Force National Guard in Tulsa, Oklahoma that December; pledged Sigma Alpha Epsilon Fraternity and attended OSU where I received a Bachelor of Science Degree in Mechanical and Aerospace Engineering in May 1994; completed requirements for the Master of Science degree with a major in Mechanical and Aerospace Engineering at Oklahoma State University in May 1997.

Experience: F-16 Jet Engine Mechanic, United States Air National Guard, 1987present; Graduate research assistant in support of NASA Dryden Flight Research Center, January 1996 - May 1997. 